
ENGINEERING 


\section{BIREFRINGENT EFFECTS IN PLASTIC OPTICS}

\section{ACHYUT ADHIKARI}

School of Mechanical and Aerospace Engineering

A thesis submitted to the Nanyang Technological University in partial fulfilment of the requirement for the degree of Doctor of Philosophy 


\section{ACKNOWLEDGEMENTS}

I would like to express my deep gratitude to my supervisor Prof Anand Krishna Asundi for his constant supervision, guidance and suggestion throughout the research. He encouraged me throughout the research and nurtured my potentialities.

I am also thankful to the technicians in both labs; centre for optical and laser engineering(COLE) and mechanics of materials (MOM). Especially, I would like to thank Mr Ang Teck Meng and Mr Koh Hai Tong who provided me with the resources needed for this project.

I acknowledge the Singapore International Graduate Award (SINGA) for providing the opportunity to conduct the research and other financial support received through NTU. Special thanks to Optics and Photonics Society of Singapore(OPSS) and International Society for Optics and Photonics (SPIE) and School of Mechanical and Aerospace Engineering which helped me attend some of very important conferences.

I would like to thank Dr Kapil Dev, Dr Muneesh Maheshwari, Ms Angela Sapkota, Mr Chongtian Huang, Mr Guan Lichao, Mr Royston Khoo regarding research materials. Also, I would like to thank my friends and batchmates Zhaomin, Sumit, Suren, Arun, Rinoj, Sharad, Sanam, Anil, Milan who helped me being there for my ups and downs. At last, but not the least I am deeply indebted to my family and friends for the constant support and encouragement. 


\section{TABLE OF CONTENTS}

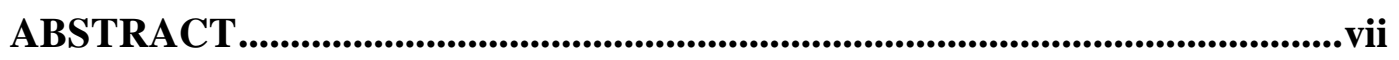

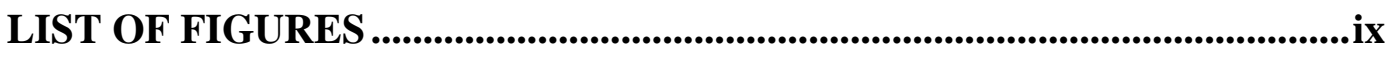

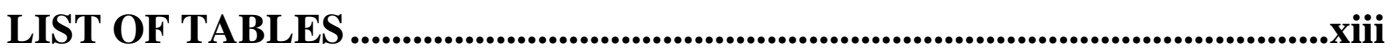

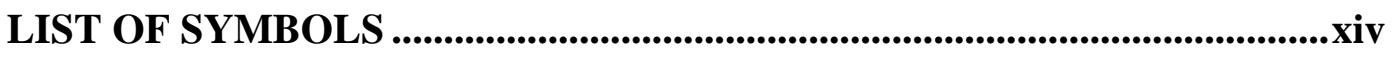

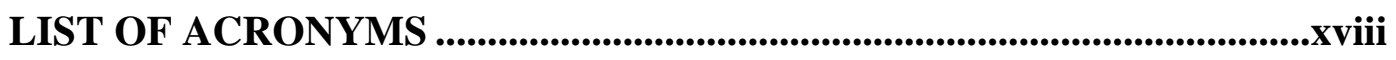

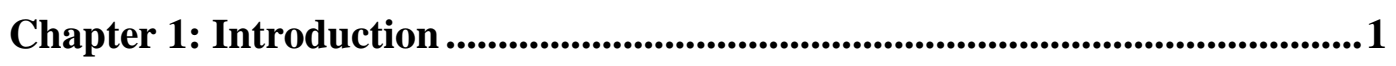

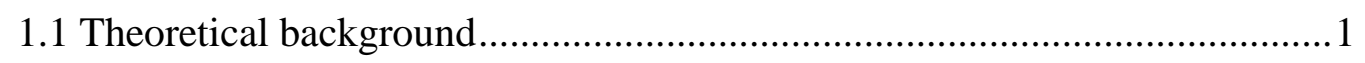

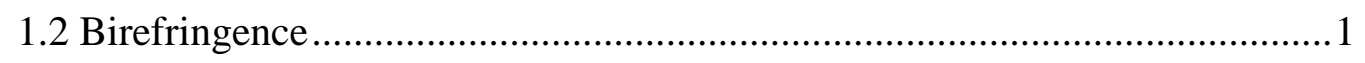

1.2.1 Effect of Birefringence in plastic optics ........................................... 2

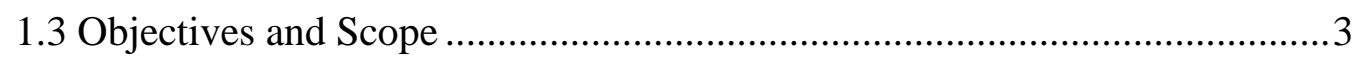

1.4 Organization of Report..................................................................... 4

Chapter 2: Literature Review and Motivation ..............................................

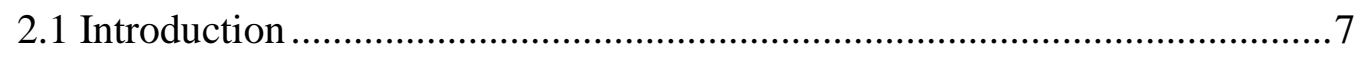

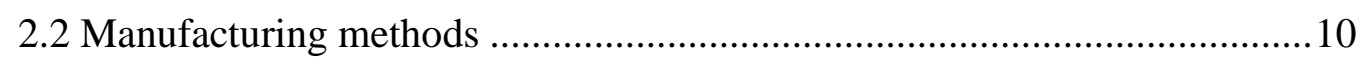

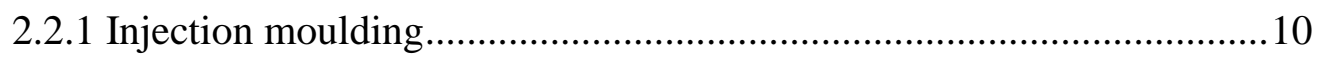

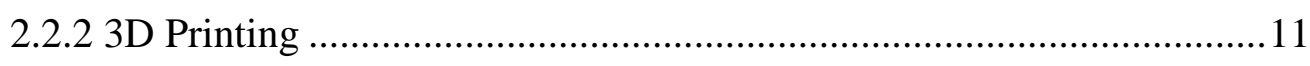

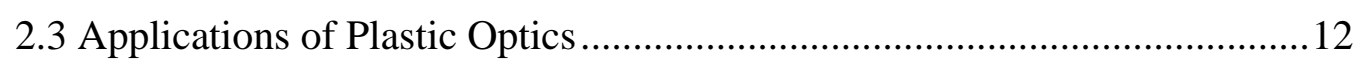

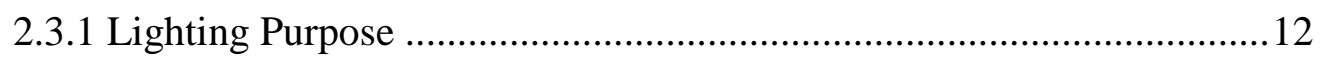

2.3.2 Communication \& Security Purpose ................................................. 13 
2.3.3 Healthcare

2.3.4 Sensors .13

2.3.5 Photovoltaics .13

2.3.6 Military .14

2.4 Birefringence. .14

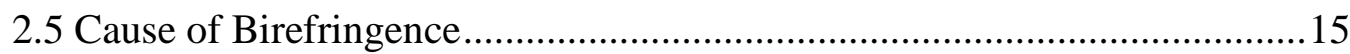

2.6 Birefringence Measurement by polariscope............................................16

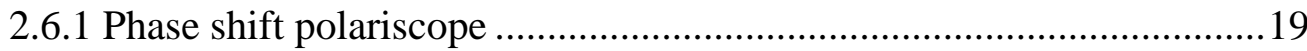

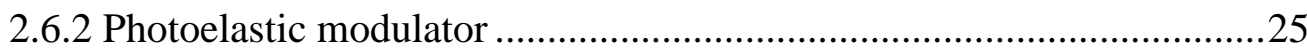

2.7 Mathematical modelling of birefringent effects in plastic optics.................30

2.7.1 Numerical Analysis .31

2.8 Effects of Birefringence in Imaging in plastic optics .36

2.9 Effects of birefringence in imaging (Experimental verification) .37

2.10 Summary .40

Chapter 3: Birefringence modelling, simulation and measurement .42

3.1 Introduction 42

3.2 Optical design and simulation via optical software .43

3.2.1 Design Specifications .43

3.2.2 Layout .44

3.2.3 Design data .45

3.2.4 Wavelength .45 
3.3 Stress-induced Birefringence modelling via Finite element analysis (ABAQUS)

3.4 Simulation results .51

3.5 Experimental results. .51

3.6 Calibration $(\mathrm{C})$ .53

3.7 Birefringence values .56

3.8 Summary .60

Chapter 4: Birefringence measurement of injection moulded optics .61

4.1 Introduction 61

4.1.1 Micro-plate material .62

4.2 Birefringence measurement by phase shifting method 63

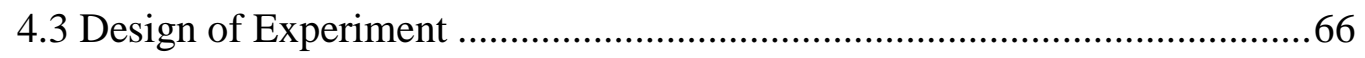

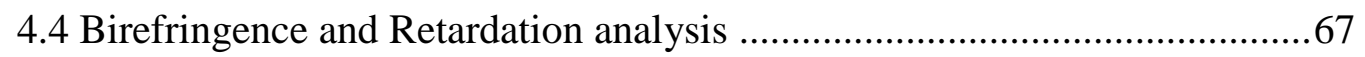

4.5 Detailed table on Birefringence and Retardation of micro-plates 69

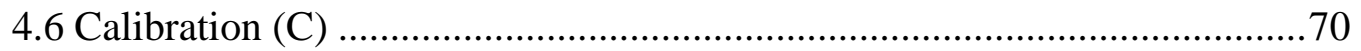

4.6.1 Compact loading machine and load cell for calibration ......................70

4.6.2 PMMA (Poly-methyl Methacrylate) …...........................................74

4.6.3 COC (Cyclic Olefin Co-polymer) …................................................76 
4.7 Microfluidic channels

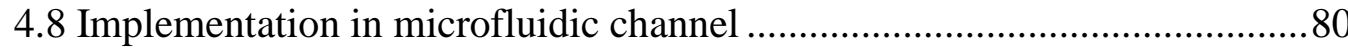

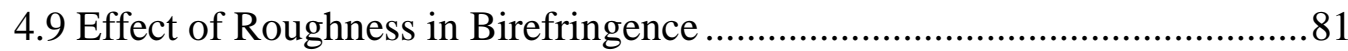

4.10 Birefringence measurement of optical windows ....................................8

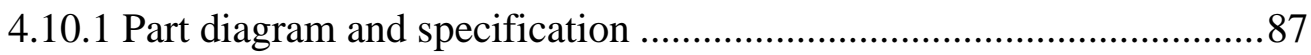

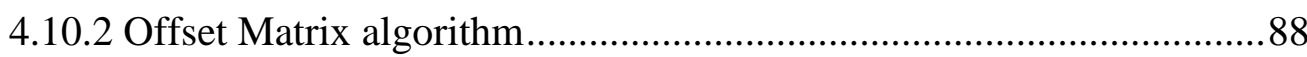

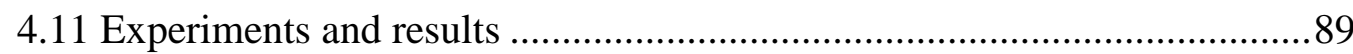

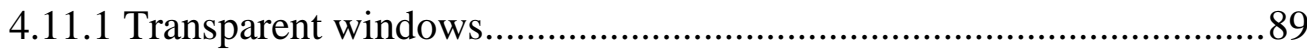

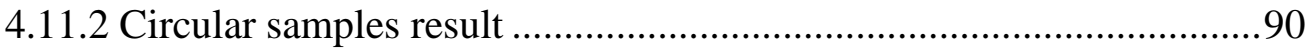

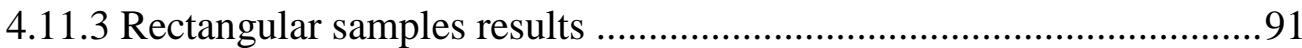

4.12 Comparison of Research method algorithm with commercial method......91

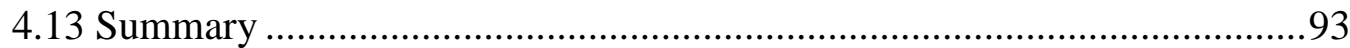

Chapter 5: Low birefringence measurement system..........................................95

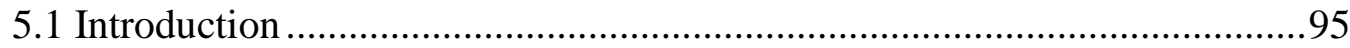

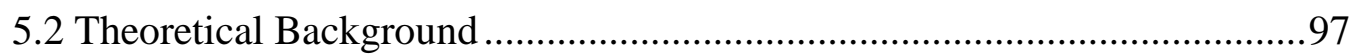

5.2.1 Jones calculus in birefringence measurement system ........................97

5.2.2 Four-step phase shifting method ……..............................................99

5.2.3 Mueller calculus in birefringence measurement system ...................100

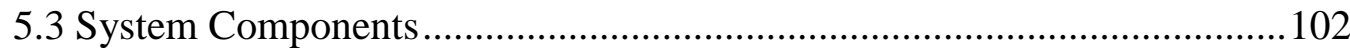

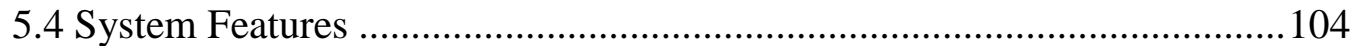

5.5 Maximum Likelihood estimation of the measurement system .................105 
5.12 Summary

Chapter 6: Conclusions and Recommendations for Future Work

6.1 Conclusions 120

6.2 Recommendations for Future Work

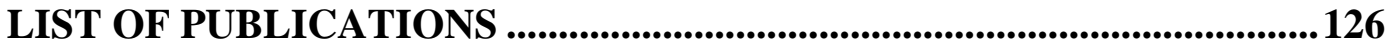

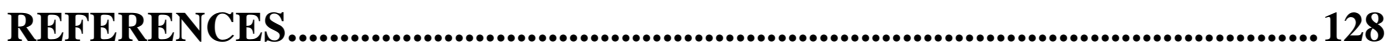

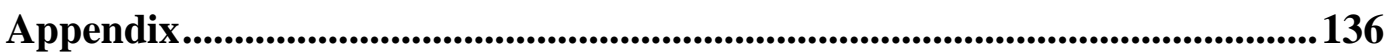

Mueller calculus: Mathematical modelling and analysis in low birefringence

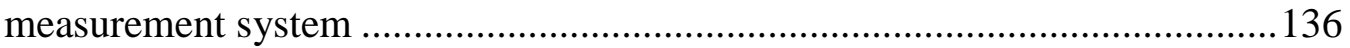

Birefringence measurement results of the optical windows ..........................138 


\begin{abstract}
Plastic optics are seeing increased usage in various imaging and non-imaging applications due to its low cost, ease of manufacturing and complex parts assembly. Non-imaging applications were the initial foray for plastic optics as the aberration and thermal effects were not important. However, recently there is an increased usage in imaging applications of plastic optics due to ease of manufacturing especially in aspheric profiles. This has brought aberrations to the forefront and more importantly birefringence effects have become critical as well. In this thesis, the birefringent effects in plastic optics are explored with a measurement purpose and its effects on imaging. This study has three main aspects. Firstly, there is a need to incorporate birefringent effects in optical design such that its effects could be modelled and simulated. This is accomplished by optical modelling software ZEMAX and simulated with finite element modelling tool ABAQUS to obtain birefringence in the optical element. The low birefringence polarimeter is designed and developed at COLE (Centre for optical and laser engineering) to measure birefringence. Enhancement of system and the calibration procedure of sample materials is discussed and analysed in detail. Theoretical, experimental and simulation of birefringence in plastic optics is carried out and their results are in good agreement.
\end{abstract}

In the second phase, the cause and measurement of birefringence are rigorously investigated. Birefringence measurement of different injection moulded optical materials like microwell plate, microfluidic channels, optical windows, etc. were 
carried out. The standard calibration procedure for microfluidic channels and optical elements is performed for qualitative and quantitative analysis.

In the third phase, the system is rigorously investigated and aimed for further improvements. Improved methodologies for automated data gathering and processing are proposed and implemented. Further, mathematical relationship and algorithm are optimised for rapid data acquisition and computation. The designed system is cheap, automatic and convenient for the prospective researchers in rapid quality testing labs.

While birefringent effects in plastic optics are thoroughly investigated, other applications which involve plastics will benefit from this study. Birefringence primarily occurs due to residual stress induced during the manufacturing processes especially in injection moulding and 3D printing method. Microfluidics and the disposable medical device is hugely affected by residual stress. Effects and techniques to reduce induced birefringence is quantitatively assessed by exploiting the polarimetric methods explained herein. 


\section{LIST OF FIGURES}

Figure 2.1: Injection moulding machine[20] 10

Figure 2.2: Schematic diagram of birefringence (left), Birefringence in the calcite material (right) [41] ...................................................... 15

Figure 2.3: Arrangement of optical elements in plane polariscope ...................20

Figure 2.4: Arrangement of optical elements in circular polariscope .23

Figure 2.5: Arrangement of optical elements based on photoelastic modulation principle .27

Figure 2.6: Projection lens composed of K birefringent elements[63] .31

Figure 2.7: Relationship between B (magnitude of birefringence) and normalized contrast[63] .36

Figure 2.8: Left (Black and white pattern subject), right (Image of the subject)...

Figure 2.9: ROI (Region of Interest) of image of the subject pattern .37

Figure 2.10: Intensity profile of ROI (Region of Interest) of image of the subject pattern .38

Figure 2.11: Reference image without sample and images on adding successive number of disks .38

Figure 2.12: Relationship between contrast values versus number of circular disks.

Figure 2.13: Relationship between contrast values versus increasing birefringence in the sample .39

Figure 3.1: Two-dimensional layout of optical setup .44

Figure 3.2: Three-dimensional layout of the optical setup employed in the simulation .46

Figure 3.3: Image rendered in the detector on passing subject through birefringent cube .46

Figure 3.4: Dispersion characteristic curve with respect to wavelength variation

Figure 3.5: Abbe number glass map in variance with refractive index for different samples [69] 
Figure 3.6: Consolidated simulation report of sample imaging through a birefringent cube

Figure 3.7: Poly-methyl methacrylate disk (Left), two-point compression loading (Right) .50

Figure 3.8: Stress values induced on specimen at different loads (simulation results)

Figure 3.9: Stress values induced on specimen at different loads (experimental results)

Figure 3.10: Stress values at different points along vertical diameter (y-axis) of specimen by different methods . .52

Figure 3.11: Compression loading in circular disk specimen ............................54

Figure 3.12: Stress distribution along vertical diameter of the specimen ...........55

Figure 3.13: Birefringence values along vertical diameter (y-axis): Left (Intrinsic birefringence), Centre (Intrinsic birefringence plot) and Right (Comparison of birefringence plot)......................................56

Figure 3.14: Birefringence values of the acrylic sample at different loads ........58

Figure 3.15: Birefringence plot along vertical diameter of the sample at increasing loads .58

Figure 3.16: Comparison of birefringence values of samples by various methods at increasing loads .58

Figure 4.1: Schematic diagram of phase shift polariscope .63

Figure 4.2: Injection moulded specimen (microplates) used in experiment .......64

Figure 4.3: Birefringence values $(\mathrm{nm} / \mathrm{cm})$ of various micro plates specimen ....67

Figure 4.4: Setup for two-point loading of circular disk in compact loading machine .70

Figure 4.5: Compact loading machine apparatus with load cell .......................71

Figure 4.6: Optical elements of phase shift polariscope measurement system...71

Figure 4.7: Compression loading in circular disk specimen for calibration purpose .72

Figure 4.8: Circular protruded acrylic specimen (left), schematic diagram (right)

Figure 4.9: Induced phase difference values at different loads in an acrylic disk 
Figure 4.10: Induced phase difference of an acrylic disk at different loads along vertical diameter...... .75

Figure 4.11: Induced phase difference of an acrylic disk after compensating intrinsic birefringence .76

Figure 4.12: Circular protruded acrylic specimen (left), schematic diagram (right)

Figure 4.13: Induced stress birefringence for cyclic olefin copolymer at increasing loads. .78

Figure 4.14: Birefringence induced in specimen along vertical diameter (with residual stress) .78

Figure 4.15: Birefringence induced in specimen along vertical diameter

(without residual stress) .78

Figure 4.16: Birefringence in microfluidic $(\mathrm{H})$ channel of top, middle and bottom with full part .80

Figure 4.17: $\mathrm{SiC}$ paper of different grit size (P180, P400, P800, P1200, P2400, P4000) .82

Figure 4.18: Intrinsic roughness of the PMMA sample .82

Figure 4.19: Roughness on sample from different grit size (i.e. P4000, P2400, P1200, P800, P400, P180) .83

Figure 4.20: Intrinsic Birefringence $(\mathrm{nm} / \mathrm{cm})$ values of the PMMA sample ......83

Figure 4.21: Birefringence $(\mathrm{nm} / \mathrm{cm})$ observed on sample by different grit size (P4000, P2400, P1200, P800, P400 and P180) .83

Figure 4.22: Roughness of the original specular sample along vertical diameter .84

Figure 4.23: Roughness of the sample after P4000 grit size grinding along vertical diameter...... .84

Figure 4.24: Roughness of the sample after P2400 grit size grinding along vertical diameter

Figure 4.25: Roughness of the sample after P1200 grit size grinding along vertical diameter .85

Figure 4.26: Roughness of the sample after $P 800$ grit size grinding along vertical diameter 
Figure 4.27: Roughness of the sample after P400 grit size grinding along vertical diameter

Figure 4.28: Roughness of the sample after P180 grit size grinding along vertical diameter.

Figure 4.29: Relationship between birefringence effects on increasing roughness of the sample .86

Figure 4.30: Setup for the measurement of two optical windows of different shape in polariscope

Figure 4.31: Left (Transparent window sample), Right (Birefringence Image). 90

Figure 4.32: Birefringence values in $\mathrm{nm} / \mathrm{cm}$ of circular window filter .90

Figure 4.33: Birefringence values in $\mathrm{nm} / \mathrm{cm}$ of rectangular window filter .91

Figure 4.34: Phase difference comparison with Hinds commercial and phase shift method .92

Figure 5.1: Low Birefringence measurement system ...................................96

Figure 5.2: Schematic diagram of Low Birefringence Polariscope (LBP) .........97

Figure 5.3: Transmission characteristics of polarizers at various wavelengths. 102

Figure 5.4: Retardance versus angle of incidence in degrees 103

Figure 5.5: Maximum likelihood estimation (Standard distribution of the sample) 105

Figure 5.6: Scatter plot of the sample data normal distribution 106

Figure 5.7: Relationship between Cramer Rao lower bound and unbiased estimator.

Figure 5.8: Phase retardation and direction of the PMMA sample by existing algorithm

Figure 5.9: Phase retardation and direction of the PMMA sample by new algorithm

Figure 5.10: Birefringence and phase view of the microfluidic channels .......116

Figure 5.11: Integrated Residual stress in Microfluidic Channels..................116

Figure 5.12: Software interface of low birefringence measurement system ....117 


\section{LIST OF TABLES}

Table 2.1: Properties of plastic optics materials .............................................. 8

Table 2.2: Comparison of plastic optics materials ............................................. 9

Table 2.3: Photoelastic coefficients of plastic optics ...........................................18

Table 3.1: Design specification of optical setup ................................................43

Table 3.2: Design specification deployed in optical setup................................45

Table 3.3: Wavelength employed in the optical simulation setup .......................45

Table 4.1: Intensity equations for phase shift polariscope ...............................63

Table 4.2: Dimensions of specimen plates ..................................................65

Table 4.3: Properties of biological microplates .................................................66

Table 4.4: Birefringence and retardation values of micro plate specimen...........69

Table 4.5: Birefringence observed in the sample on increasing Roughness $\left(R_{q}\right) .86$

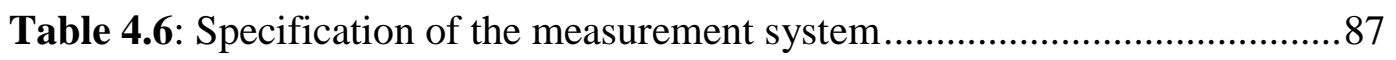

Table 5.1: Phase shifting in low birefringence polariscope ..............................99 


\section{LIST OF SYMBOLS}

\section{$\underline{\text { Symbols }}$}

$f_{\sigma}$

${ }^{0} \mathrm{C}$

$\mathrm{A}_{\mathrm{w}}$

B

C

$\mathrm{C}_{\mathrm{ij}}$

D

DOP

$\varepsilon$

E

f

$f$

\section{$\underline{\text { Definitions }}$}

Fringe constant

Degree celsius

Aperture diameter of window

Birefringence

Photoelastic coefficient

Dielectric impermeability tensor

Diameter of specimen

Degree of polarization

Degree of coherence

Young's modulus

Frequency

Orientation coefficient 
Shear modulus

$\mathrm{Hz}$ Hertz

J

Bessel amplitude function of retardation

$J$

Jones matrix

M

Molecular weight per unit

$\mathrm{M}_{\mathrm{k}}$

Jones matrix

$\mathrm{m}_{\mathrm{xy}}$

Polarization component

n

Mean refractive index

$\mathrm{n}_{1}$

Refractive index in medium 1

$\mathrm{n}_{2}$

Refractive index in medium 2

$\mathrm{N}_{\mathrm{A}}$

Avogadro's number

NA

Numerical aperture

$\mathrm{P}$

Applied load

$\mathrm{P}_{\mathrm{w}}$

Uniform load

$\mathrm{p}_{\mathrm{x}}$

Polarization state at $\mathrm{x}$ axis

q

Stress optic coefficient 
$\mathrm{Q}_{\mathrm{k}}$

$\mathrm{R}$

$\mathrm{t}$

V

Z

$\alpha$

$\beta$

$\delta$

$\Delta$

$\Delta a$

$\Delta \mathrm{n}$

$\Delta \mathrm{n}_{\mathrm{o}}$

$\Delta \mathrm{n}_{\mathrm{s}}$

$\theta$

$\lambda$

$\pi$
Wavefront deviation

Radius of curvature

Thickness of the specimen

Voltage

Path length

Angular position of analyser

Retardation direction

Retardation

Retardation Intensity value

Polarization difference

Birefringence

Intrinsic birefringence

Stress birefringence

Optical axis direction

Wavelength

Pi 
Density

$\sigma$

$\omega$
Stress tensor

Azimuthal angle 


\section{LIST OF ACRONYMS}

$3 \mathrm{D}$

Three Dimensional

ABS Acrylonitrile Butadiene Styrene

$\mathrm{AC}$

Alternate Current

AOI

Angle of Incidence

AR

Anti Reflective

ASTM American Standard Test Method

BK7 Borosilicate Glass

BMS Birefringence Measurement System

BWD Back Working Distance

CAD Computer Aided Drawing

CAM Computer Aided Manufacturing

CCD Charged Coupled Device

CD Compact Disc

CDRH Centre for Devices and Radiological Health 
CNC Computer Numerical Control

COC Cyclic Olefin Copolymer

COP Cyclic Olefin Polymer

CPV Concentrated Photo Voltaic

CTE Coefficient of Thermal Expansion

DC Digital Current

DNA Deoxy Ribo-Nucleic Acid

DVD Digital Versatile Disk

FDM $\quad$ Fused Deposition Melting

FEM Finite Element Modelling

FLIR Forward Looking Infra-Red

FOV Field of View

FTIR Far Transmission Infra-Red Radiometer

GUI Guided User Interface

HDPE High- Density Poly Ethylene

HMD Head Mounted Display 
IR

Infra-Red

$\mathrm{KHz}$

Kilo Hertz

LBP

Low Birefringence Polariscope

LCD

Liquid Crystal Display

LCP Liquid Crystal Polymer

LED Light Emitting Diode

MATLAB Matrix Laboratory

NA Numerical Aperture

PC Polycarbonate

PEM Photoelastic Modulator

PET Polyethylene Terephthalate

PLA Poly Lactic Acid

PMMA Poly Methyl Methacrylate

PPM Parts Per Million

PPSU Poly Phenyl Sulfone 
Poly Sulfones

PSP

Phase Shift Polariscope

RGB

Red Green Blue

RMS Root Mean Square

SIMTech Singapore Institute of Manufacturing Technology

USB Universal Serial Bus

WD Working Distance 


\section{Chapter 1: Introduction}

\subsection{Theoretical background}

Plastic optics [1] are increasingly finding applications in various optical systems because of its affordable cost and ease of complex shape manufacturing. Applications in [2] diverse fields are lighting industry, mobile communications, security, healthcare, photovoltaic, sensors and many others. Lower cost, higher impact resistance, lighter weight and more configuration possibilities for varying application are the significant aspects of plastic optics[3]. As aspherical lenses can be designed with more configuration flexibility, manufactured parts will have reduced weight and cost. Moreover, different refractive indices of plastic materials are available generating low-grade to high-grade transmissive plastic optics incomparable to high-grade crown glasses. The injection moulding process is ideal for producing a large volume of economical plastic optics parts. Multicavity moulds allow a low-cost manufacturing process attributed to its mass scale production and inexpensive products. Plastic optics with residual stress induced during manufacturing engender birefringent effects detrimental in imaging applications $[4,5]$. It has reduced heat tolerance than glass, hence cannot be used for extreme temperature applications.

\subsection{Birefringence}

Birefringence or double refraction is an optical property which causes the material to be optically anisotropic. In plastic materials, [6,7] mechanical stress induces an optically isotropic material to optically anisotropic (birefringent) material. This causes a single ray to split into two rays travelling in different directions [8] and velocity owed to their change in relative refractive indices with respect to air. 
Optical anisotropic (or birefringent) materials are characterised by two refractive indices in two perpendicular directions. Hence when polarised light enters the material at the same angle of incidence, a component of the incident radiation whose polarisation is perpendicular to the optical axis will be reflected according to the standard law of refraction with refractive index $\mathrm{n}_{1}$, and the next polarization component so called extraordinary axis $\mathrm{n}_{2}$ will refract at a different angle determined by material anisotropic behaviour known as birefringence [9],

$$
\Delta \mathrm{n}=\mathrm{n}_{2}-\mathrm{n}_{1}
$$

Birefringence is found in anisotropic materials due to following reasons:

Stress-induced birefringence[10] occurs when isotropic material possess residual stress such that anisotropy will propagate during stretch or bent.

Due to self or forced alignment on thin films of amphiphilic molecules, lipids, surfactants or liquid crystals[11, 12].

\subsubsection{Effect of Birefringence in plastic optics}

All plastic materials including optical systems for instances; camera lenses, moulded plastics and microfluidic channels have intrinsic birefringence. Intrinsic birefringence in material affects wavefront [13] formations degrading imaging performance[14]. It is almost impossible to attain zero birefringence of the material known as aberration-free imaging (birefringence free) in the visible wavelength region. Birefringence effects in imaging can't be neglected since it deteriorates image contrast. Birefringence causes wavefront deviation which is inversely proportional to the square of the wavelength [15]. It hugely affects the irradiance [16] and phase pattern of the beam during transmission. Polarisation 
aberration occurs in the birefringent material which modifies the polarisation phase of the transmitted light. Hence, birefringence effects in imaging should be determined through mathematical modelling, simulation and analysis of the material before application.

\subsection{Objectives and Scope}

The aim is to study the effects of birefringence in plastic optics for imaging and other applications.

\section{Scope}

The research scope includes the following plans to be accomplished.

$>$ Plastic optics manufactured by injection moulding or 3D printing will only be considered.

$>$ Study the degradation in applications such as multi-well plate imaging, optical windows and microfluidic channel imaging.

Improvement of polarimetry for low-level birefringence measurement over the whole field with high resolution and reduced distortion.

An automated system designed for birefringence measurement system with reduced measurement time and increased computational speed.

A possible implementation of the developed system in IR bandwidth which is vital in quality testing of Silicon $(\mathrm{Si})$ wafer (a thin slice of semiconductor material used in the fabrication of integrated circuits and photovoltaics) at a reduced cost. 


\subsection{Organization of Report}

The work presented in this thesis focuses on birefringent effects in plastic optics with a tuneable light source and other advanced features. The primary emphasis of this report is to investigate various plastic optics affected by their intrinsic birefringence. This report is organised into six chapters as follows.

Following a brief introduction of plastic optics and its effects in imaging, Chapter 2 presents various plastic optics with its birefringence properties. Different existing manufacturing methods and their limitations are discussed. Plastic optics parts and their application in diverse fields are briefly reviewed. Birefringence terminology is well described along with its causes and effects on plastic materials. Mathematical modelling of birefringent effects on plastic optics and its detailed analysis is presented. Birefringence measurement system is entirely investigated based on its working principles and methods. Different birefringent systems are compared with their remarkable features and drawbacks.

In Chapter 3, a birefringent phenomenon from source is modelled using a commercial software ZEMAX and complete analysis is performed from the image acquired by the detector. Since the imaging software, ZEMAX lacks stress analysis module, finite element modelling software ABAQUS is deployed to obtain the stress-induced birefringence. The birefringence values induced due to simulated stress in the modelling software and mathematical calculation are in good agreement.

In Chapter 4, various injection moulded samples were tested and calibrated for validation and verification of the results. Microwells, extensively used in 
biological labs for qualitative and quantitative assessments are of critical concern. Birefringence assessment on the microwells inspects defective parts facilitating selection procedure. Rheological samples from SIMtech (Singapore Institute of Manufacturing Technology) were tested for material uniformities. The tested samples are identical and have same photoelastic coefficient as provided in optical handbooks. These samples are rigorously tested and verified for further production of optical samples from same material particularly microfluidic channels. Thereafter, birefringence calibration of microfluidic channels is carried out. Microfluidic channels are made up of polymethyl methacrylate (PMMA) and cyclic olefin copolymer (COC). Microfluidic samples from SIMTech were inspected to measure their birefringence values. Any deviation due to birefringence at different points will render altered image thus providing false scientific results. The circular samples made from the same injection moulding machine are calibrated to get their respective photoelastic values. Further, this photoelastic values $\left(5.14 \times 10^{-12} \mathrm{~m}^{2} / \mathrm{N}\right.$ for PMMA and $7.19 \times 10^{-12} \mathrm{~m}^{2} / \mathrm{N}$ for COC) were implemented in microfluidic channels to obtain birefringence values. This measurement is vital in identifying local and global birefringence in defective areas applicable in quality control for delicate diagnosis of biological specimens. Similarly, qualitative and quantitative inspection of optical windows were carried out by assessing their birefringence values. It is vital for quality control and crucial in sensing applications. Birefringence in the optical windows deteriorates signals and disrupts the entire conveyor system in supermarkets. Further, the results from our system is compared with the prevalent commercial system and the results were found to be in good agreement, henceforth validated and verified. 
In Chapter 5, Automated Birefringence testing system known as low birefringence measurement system is developed. The existing system was modelled on Jones matrix whereas new mathematical approach is modelled on Mueller matrix. New mathematical approach implemented on this system will lead to more information about the sample i.e. diatttenuation (intensity transmittance), polarizance and retardation. The system is of high resolution with wide FOV (field of view), fully automated and vital for mass testing of the specimen. This system has bright commercial scope and has been used in SIMTech and Fluidigm for mass inspection of the sample specimens. Moreover, a new algorithm for RGB photoelasticity is implemented and its results are validated with the existing system. Existing system uses polariser, analyser and quarter wave plate whereas the new algorithm eliminates quarter wave plate which reduces the cost and complexities associated with the system. Thus, this system will likely to have good prospects in industrial and scientific research laboratories.

Chapter 6 concludes the entire work performed in this thesis. A possible application of the research conducted has been presented. Moreover, future scope of the report is also discussed in this chapter. 


\section{Chapter 2: Literature Review and Motivation}

\subsection{Introduction}

The manufacturing of plastic optics is on advancement due to its wide application in various fields. Plastic optics are extensively used in lighting, communication, healthcare, photovoltaic and military purposes[17]. Here, we considered plastic optics that is transparent and utilised for imaging and sensing. Earlier, conventional materials particularly glass crystals were used but have less stability to temperature deformation. Thus, where stability is an essential factor, plastic optics plays key role. Moreover, it can be manufactured in huge volumes with minimal cost and can be shaped into different configurations with adequate flexibility. Among the transparent plastic optics, polymethyl methacrylate (PMMA) and polycarbonate (PC) possess excellent transparency at minimum cost, hence widely used for various purposes. Moreover, alicyclic acrylates and cycloolefin polymers (COPs) are also used subjected to the optical characteristics required. Multi-cavity moulding eases fast production cycle at a cheaper cost and varying configuration. With being cost efficient in mass manufacturing, mechanical parts are also integrated with plastic parts during production. Higher accuracy and precision can be achieved by mass production of plastic parts. Plastic parts can be piled up without alignment and reference problem. By optomechanical design procedure, fewer parts and components are required. On using mounting rigs, spacers and integration parts, complex plastic optics can be manufactured at reduced time and cost. Complicated shapes are produced by advanced tooling method in injection moulding process. In addition to mass production, lightweight product is an impressive feature of plastic optics. Due to this flexibility, plastic optics have good prospects in bio-sensing and 
biotechnology. Along with these vital elements of plastic optics, there are a few drawbacks regarding durability and environmental stability.

Plastic optics have limited temperature range at which they can properly function. Water absorption, thermal expansion and change in refractive index are significant problems in injection moulded optics. However, in most cases, the advantages of injection moulded optics outweigh the disadvantages. Thus, injection moulding of optical components represents the state of the art for manufacturing high volume of plastic optics. For transparent plastic optics, poly-methyl methacrylate (PMMA) and polycarbonate (PC) have excellent transparency and are widely used. Moreover, alicyclic acrylates, optical polyester (O-PET), polysulfones and cycloolefin polymers (COPs) with different optical properties have been manufactured and are in research phase for their material improvement. With the rapid advancement of technology, complexity in shapes of plastic optics is in high demand in advanced manufacturing. Complicated shapes and various configurations have been possible due to advancement in injection moulding method. The properties of standard plastic optics materials are represented in Table 2.1 along with their comparison in Table 2.2.

Table of properties of plastic optics materials[18]

\begin{tabular}{|l|l|r|r|r|r|r|r|}
\hline & Unit & \multicolumn{5}{|c|}{ Polymer material } \\
\hline Property & & PMMA & PC & COP & COC & PET & PSU \\
\hline Transmittance & & 92.5 & 89 & 92 & 90 & 90 & 84 \\
\hline Refractive Index & & 1.49 & 1.585 & 1.53 & 1.54 & 1.607 & 1.634 \\
\hline Abbe number & & 58 & 30 & 56 & 56 & 52 & 23 \\
\hline Coefficient of thermal expansion & ${ }^{\circ} \mathrm{C}$ & 106 & 147 & 139 & 135 & 156 & 190 \\
\hline Birefringence & $\mathrm{nm}$ & -0.0043 & 0.106 & 0.141 & 0.203 & 0.105 & 0.121 \\
\hline
\end{tabular}

Table 2.1: Properties of plastic optics materials 


\section{Comparison of plastic optics}

\begin{tabular}{|l|r|r|r|r|r|r|r|}
\hline Property & \multicolumn{7}{|c|}{ Polymer material } \\
\hline Optical & PMMA & PC & COP & COC & PET & PSU \\
\hline Light transmittance & 5 & 3 & 5 & 4 & 3 & 5 \\
\hline Birefringence & 4 & 2 & 3 & 5 & 1 & 5 \\
\hline Refractive Index & low & high & medium & medium & high & high \\
\hline Abbe number & high & low & high & high & low & high \\
\hline Stability & & & & & & \\
\hline Water absorption & 1 & 3 & 5 & 5 & 2 & 5 \\
\hline Coefficient of Linear Expansion & 3 & 3 & 3 & 3 & 3 & 5 \\
\hline Deflection Temperature & 3 & 4 & 4 & 4 & 3 & 5 \\
\hline Moldability & & & & & & \\
\hline Flowability/Injection molding & 4 & 4 & 4 & 5 & 2 & 3 \\
\hline
\end{tabular}

Table 2.2: Comparison of plastic optics materials

PMMA = Poly Methyl Methacrylate

$\mathrm{PC}=$ Polycarbonate

$\mathrm{COP}=$ Cyclic Olefin Polymer

$\mathrm{COC}=$ Cyclic Olefin Copolymer

PET = Polyethylene terephthalate

PSU = Poly-sulfone

The common problems from these materials are the undesired birefringence induced during manufacturing which degrades the desired performance. 


\subsection{Manufacturing methods}

\subsubsection{Injection moulding}

Injection moulding process is carried out using single and multi-cavity moulds. For complex manufacturing of plastic optics, multi-cavity moulds are used. It optimises part cost, tooling complexity and precision level. Injection moulding method represents current state of the art enabling mass production with minimum unit cost. The injection moulding assembly consists of clamping unit and an injection unit[19] as shown in Figure 2.1. Plastic pellets are placed in the hopper which is previously dried with a desiccant drying system, and the movable platen is moved forward to close the moulding part. The high pressure is created in the moulding part with moulding materials in a molten state that is cooled and solidified. It gives the shape of the insert cavity once mould opens after brief cooling. The ejection mechanism on clamp separates optics from the mould. Mould inserts are repetitively used and are fabricated of high precision and accuracy. Ultraprecision machining procedure fabricates aluminium mould inserts via single point diamond turning with high precision and accuracy. Mostly, inserts are manufactured from tool steel with an inner Nickel (Ni) plating. To obtain the smooth surface of the roughness in $\mathrm{nm}$, diamond turning is applied on the inner surface.

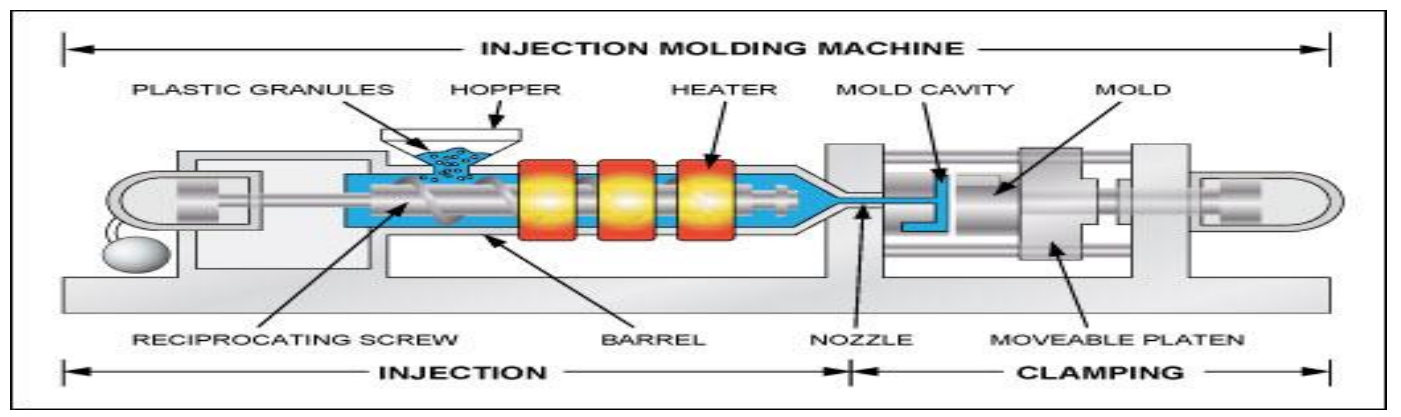

Figure 2.1: Injection moulding machine[20] 


\subsubsection{D Printing}

The digital geometry input is provided to a 3D (three dimensional) printing CNC (Computerized Numerical Control) machine via an inbuilt modelling and simulation software. 3D printing machine produces specimens from raw materials with an accuracy of micron level at promising ease and flexibility. The required samples are printed using three major techniques [21-23]:

2.2.2.1 Fused deposition modelling: It is a commonly used method in which the part is produced by extruding small beads of raw material through the extrusion head. Basically, raw materials are made up of thermoplastic material or plastic pellets which harden immediately to form layers. Stepper motors or servo motors are employed to move the extrusion head to adjust the flow. The extrusion head can be moved both in horizontal and vertical directions via computer aided manufacturing (CAM) software. Different polymers are used for printing for e.g. acrylonitrile butadiene styrene (ABS), polycarbonate (PC), polylactic acid (PLA), high-density polyethene (HDPE) and polyphenyl sulfone (PPSU).

2.2.2.2 Stereolithography: It is employed for manufacturing models, patterns, prototypes by applying Ultra-violet (UV) laser on the photoreactive resin. A vat of liquid polymer is exposed to controlled light solidifying into photo-polymerized material. The exposed liquid polymer hardens and is drained from the vat, yielding the solid model.

2.2.2.3 Selective laser sintering: This process is applicable for manufacturing solid objects from powders. The powder can be metal, plastic, ceramic or glass material. It utilises a pulsed laser to draw the desired cross-section of the material. 
The powder coalesces to form the desired specimen using a layer by layer method which requires a powerful laser.

Besides these, there are other manufacturing methods for e.g. cladding or laser powder forming, laminated object manufacturing, laser cutting and CNC milling which is beyond the scope of this thesis.

\subsection{Applications of Plastic Optics}

Injection moulded plastic optics have extensive use in the fields of the lighting industry, mobile communications, security, healthcare, photovoltaics, sensors and various components[24]. The main reason of its usage is because of its low cost, optomechanical integration, higher impact resistance, lighter weight and more configuration possibilities. Moreover, different refractive index of plastic optics materials can be manufactured from low-grade to high-grade transmittance incomparable to that of high-grade crown glasses.

2.3.1 Lighting Purpose: Lighting industry has shifted from incandescent to optimised LED (Light Emitting Diode) lighting system[25]. The radiation pattern of light has shifted to more hemispheres compared to conventional incandescent light sources which are deployed on LED sources. It is two step approach; one is a primary optical element in front of the emitting diode for making boundaries on radiation angle and next is a secondary optical element to shape the light into the desired form, both of which are injection moulded plastics[26]. Another advantage of injection moulded optics is freeform optics which can be obtained by the advanced manufacturing technology. 
2.3.2 Communication \& Security Purpose: Plastic optics have been extensively used for mobile communication and entertainment. CD (Compact Disc), DVD (Digital versatile disk) player, camera, phone flash, a Head-mounted display (HMD) and many other materials have been manufactured via injection moulding method. Surveillance cameras with lenses, iris scanners, fingerprint readers, moulded optical parts are widely used because of its low cost and integration capabilities.

2.3.3 Healthcare: Syringes, microplates, test-tube and various medical devices are cheaply manufactured by injection moulding method. Moreover, we can integrate injection moulded plastic parts, for instance, spectacle with a polycarbonate frame and plastic lens together[27].

2.3.4 Sensors: Most of the sensors used in our daily life are manufactured via injection moulded plastics. It is due to its flexible integration capabilities[28] and mass production at low-cost. For instance, barcode scanning device, optical mouse, construction theodolite, automation sensors in light gates, optical toys, vision systems and micro lens arrays are commonly used sensors.

2.3.5 Photovoltaics: Concentrated photovoltaics manufactured via injection moulding method accumulate maximum solar radiation which is laid above photovoltaic cells. Concentrated photo voltaic (CPV) component used in conjunction with photovoltaics manufactured at low cost collects maximum solar energy with high efficiency[29, 30]. Most of the CPVs[29, 31] used today are made up of the primary optical element (Fresnel lens) [32-37] and secondary optical element i.e. compound parabolic concentrator. Similarly, it can be utilised 
for LED illumination system opposite to that of concentration, for diverging the light source.

2.3.6 Military: Night vision devices made up of injection moulded plastic optics are lighter, sophisticated and inexpensive compared to glass[38] materials. The plastic element is approximately a factor of two to five times lighter than glass. Thus, injection moulded plastic optics are highly recommended as a portable optical element in military purpose.

\subsection{Birefringence}

Birefringence is exhibited when a bundle of natural light transmits through an anisotropic medium. It is intrinsic material property because of the nonuniformities of the refractive index in the material. Birefringence is qualitatively and quantitatively analysed by transmitting polarised light through it. Optical glasses are ideally isotropic under stress-free condition. During manufacturing, the material deforms altering its microstructure and different particle densities along different axes [39]. As the propagation of the light velocity depends on the density of the material, the changes in the microstructure will result in different light velocities[40] along the axes as illustrated in Figure 2.2. Hence, the material will exhibit birefringence under stress and this effect is known as stress birefringence. The material exhibits phase difference $(\Delta)$ during its transmission due to its anisotropic material characteristics. Due to phase difference, optical retardation is observed in the sample which is given by,

Optical Retardation (R) induced in the sample is given by, 


$$
\mathrm{R}(\mathrm{nm})=\frac{\Delta \times \lambda}{2 \pi}
$$

where $\Delta$ is phase difference induced in the sample and $\lambda$ is the wavelength of the light source in $\mathrm{nm}$. The birefringence observed in the material is normalised and is given by,

$$
\text { Birefringence } B(\mathrm{~nm} / \mathrm{cm})=\frac{R}{t}
$$

where $\mathrm{R}$ is the optical retardance in $\mathrm{nm}$ and $\mathrm{t}$ is the thickness of the sample in $\mathrm{cm}$.
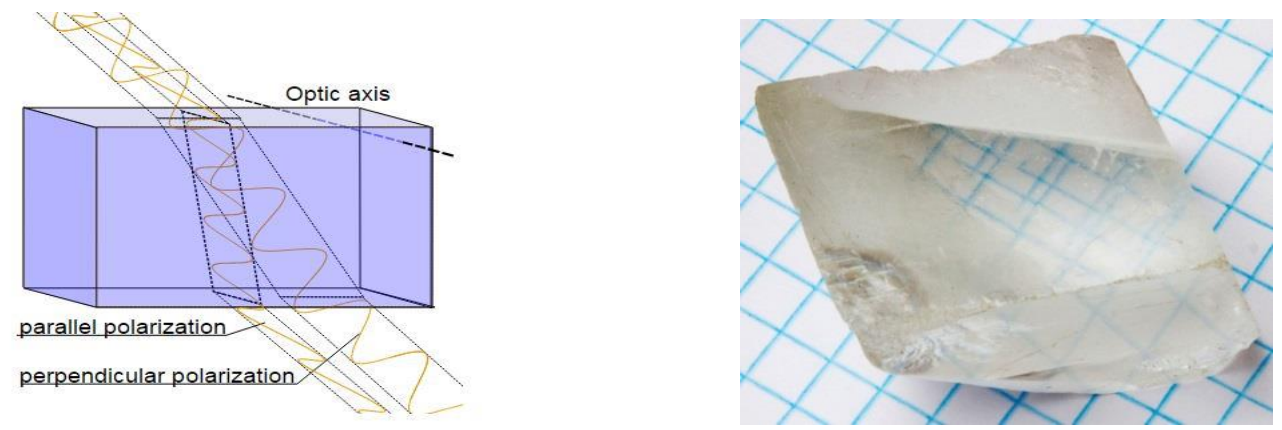

Figure 2.2: Schematic diagram of birefringence (left), Birefringence in the calcite material (right) [41]

\subsection{Cause of Birefringence}

In a material when a light wave passes, the velocity of light wave varies between two orthogonally arranged vibrating surfaces known as birefringence. Birefringence in the lens is undesirable, so the null image is formed around the focal area of the lens. Birefringence is induced in polymer due to following reasons:

Orientational birefringence which is given by,

$$
\Delta n=f \Delta n_{o}
$$


where $f$ is the directional constant, $n$ is mean refractive index, and $\Delta \mathrm{n}_{0}$ is the intrinsic birefringence specified by,

$$
\Delta n_{o}=\frac{2 \pi\left(n^{2}+2\right)^{2} \rho N_{A} \Delta \alpha}{9 n M}
$$

Equation (2.4) is the intrinsic birefringence of the optical element, where $\rho$ is density, $M$ is the molecular weight per unit, $N_{A}$ is Avogadro's number and $\Delta \alpha$ is the polarisation difference.

Stress birefringence is given by,

$$
\Delta n_{s}=C \sigma
$$

where $C$ is the photoelastic constant and $\sigma$ is the induced stress of the material. Plastic optics have two characteristic constants i.e. intrinsic birefringence and photoelastic coefficient. To minimise the birefringence in plastic optics, variation in refractive index is reduced by minimising the stress based on equation (2.5). Similarly, synthesizing alicyclic structures into the parent chain helps in reduction of birefringence[18]. The combination of structures with positive and negative birefringence minimizes the stress birefringence of the material.

\subsection{Birefringence Measurement by polariscope}

Polariscope utilises photoelasticity principle to measure the birefringence present in the material. It is an instrument to measure the relative retardation or phase differences when polarised light passes through a photoelastic sample. The optical distance travelled by the optical wave encounters a slight offset in the specimen known as phase difference due to material non-uniformities. Thus, polarised light 
traversing through the same medium is split into two components of different refractive indices and different velocities. This phenomenon is known as double refraction or birefringence that can be observed in the birefringent specimen. When an incident polarised light passes through the birefringent surface, the relative phase difference between the two components is given by,

$$
\delta=2 \pi\left(n_{1}-n_{2}\right) d / \lambda
$$

where $d$ is the distance travelled by rays in the birefringent specimen, $n_{1}$ and $n_{2}$ are their respective refractive indices. The linear phase difference or also known as optical retardation between the two waves is given by,

$$
e=\frac{\delta \lambda}{2 \pi}=d\left(n_{1}-n_{2}\right)
$$

Relative retardation is defined by,

$$
R=\frac{e}{\lambda}=\frac{\delta}{2 \pi}=\frac{d}{\lambda}\left(n_{1}-n_{2}\right)
$$

From the above equation, normalised birefringence of the system is obtained as,

$$
n_{1}-n_{2}=\frac{R}{t}=\frac{\lambda \alpha}{2 \pi d t}
$$

Since birefringence is proportional to the residual stress of the material we get,

$$
B r=n_{1}-n_{2}=C\left(\sigma_{1}-\sigma_{2}\right)
$$

The principal stress difference of the material is calculated by,

$$
\left(\sigma_{1}-\sigma_{2}\right)=\frac{B r}{C}=\frac{n_{1}-n_{2}}{C}
$$


Where $n_{1}-n_{2}$ is the change in refractive index of the specimen, $C$ is the photoelastic coefficient also known as relative stress optic coefficient expressed in terms of Brewster $\left(1\right.$ Brewster $=10^{-13} \mathrm{~cm}^{2} /$ dyne $)$ of the material, and $\left(\sigma_{1}-\sigma_{2}\right)$ is the difference of stress along principal axes of the optical specimen. The photoelastic coefficient[42] of the plastic specimen is provided in Table 2.3:

\begin{tabular}{|l|r|}
\hline Polymer material & Photoelastic Coefficient $(\mathrm{C})\left(\times 10^{-13} \mathrm{~cm}^{2} /\right.$ dyne $)$ \\
\hline PMMA & -6 \\
\hline PC & 72 \\
\hline COP & 6.5 \\
\hline PS & -55 \\
\hline
\end{tabular}

Table 2.3: Photoelastic coefficients of plastic optics

In photoelastic materials, the material is birefringent when subjected to loads or stresses known as stress-induced birefringence. Thus, on exploiting the photoelastic characteristic of the specimen[43], birefringence[44] property of the material can be assessed by polariscope[45]. There are various developments in polariscope; we will discuss each of them briefly. Photoelastic modulator principle works on the point to point measurement method with low resolution and longer acquisition time. Furthermore, pixelated polarisation camera has been developed $[46,47]$ that can measure the whole field on a single shot. It is modified polarisation interferometer which captures four simultaneously phaseshifted interferograms; thus, environmental disturbance, air turbulence and mechanical vibration is avoided. It has advantages over the conventional phase shifting method such as the snapshot and motionless features. On the other hand, 
fixed pattern noise (FPN), photon response non-uniformity (PRNU), pixelated image and instantaneous field of view (IFOV) error which significantly deteriorates the performance of the pixelated polarisation camera. Therefore, we confine our system with an improved phase shifting method for low noise and enhanced sensitivity with improved accuracy.

\subsubsection{Phase shift polariscope}

Polariscope comprises of multiple elements, the first or the fixed element is known as a polarizer along with quarter wave plate, the second element is a specimen stage, the third element is an analyser which is rotated to capture the phase shifted images and the final element is an image processing system. The working principle is that as polarised light travels through a transparent material; it experiences birefringence proportional to the amount of stress[48, 49]. There has been a gradual advancement in phase shifting polariscope. Plane polariscope provides birefringence values along with fast axis direction values. Fast axis direction values are undesired values in the polariscope and renders noise in the image. Fast axis direction values are compensated when quarter wave plates are deployed in the system by yielding phase difference results with enhanced sensitivity. In plane and circular polariscope, there are dark field images which will have obviously less information on their image. To overcome this limitation, Senarmont and Tardy[50] have devised compensation method to obtain bright uniform circularly polarised light. This method further enhanced phase difference values with better accuracy and precision. 


\subsubsection{Plane polariscope}

It consists of two polarizers arranged in a dark field where the optical specimen is inserted between them as shown in Figure 2.3. Once the specimen is applied with force, it functions as a birefringent plate and its direction and retardation values are measured. The reference axis is taken on objects $\mathrm{x}$-axis and the retardation values $(\delta)$ and its directions $(\beta)$ are computed. The light rays traverse through the first element polarizer and the specimen followed by the second element as an analyser.

\section{Mathematical modelling on Jones calculus}

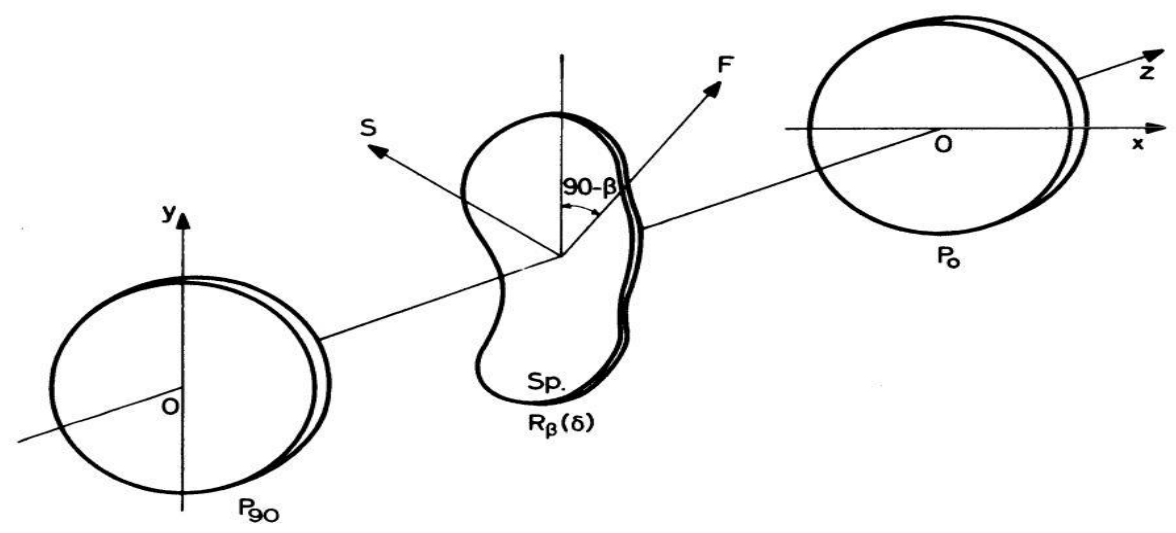

Figure 2.3: Arrangement of optical elements in plane polariscope

The light emerging from the polarizer that is linearly polarised and aligned on vertical axis of normalised Jones vector magnitude ' $a$ ' is given by,

$$
a=\left[\begin{array}{l}
0 \\
1
\end{array}\right]
$$

Birefringent plate or sample with retardation $\delta$ is represented by $\mathrm{R}_{\beta}(\delta)$ of $2 \times 2$ Jones matrix, where fast axis subtends an angle $\beta$ with the origin inclined to $\mathrm{x}$-axis is given by, 


$$
R_{\beta}=\left[\begin{array}{ll}
e^{i \delta} \cos ^{2} \beta+\sin ^{2} \beta & \left(e^{i \delta}-1\right) \sin \beta \cos \beta \\
\left(e^{i \delta}-1\right) \sin \beta \cos \beta & e^{i \delta} \sin ^{2} \beta+\cos ^{2} \beta
\end{array}\right]
$$

A second linear polarizer also known as analyser is at crossed optical axis (horizontal) and its Jones matrix is given by,

$$
P_{0}=\left[\begin{array}{ll}
1 & 0 \\
0 & 0
\end{array}\right]
$$

On computing the Jones calculus, the output vector a' leaving from the analyser is given by,

$$
a^{\prime}=P_{0} R_{\beta}(\delta) a
$$

Implementing the above equation, we obtain

$$
\begin{gathered}
a^{\prime}=\left[\begin{array}{ll}
1 & 0 \\
0 & 0
\end{array}\right]\left[\begin{array}{ll}
e^{i \delta} \cos ^{2} \beta+\sin ^{2} \beta & \left(e^{i \delta}-1\right) \sin \beta \cos \beta \\
\left(e^{i \delta}-1\right) \sin \beta \cos \beta & e^{i \delta} \sin ^{2} \beta+\cos ^{2} \beta
\end{array}\right]\left[\begin{array}{l}
0 \\
1
\end{array}\right] \\
=\left[\begin{array}{c}
\left(e^{i \delta}-1\right) \sin \beta \cos \beta \\
0
\end{array}\right]
\end{gathered}
$$

The intensity collected at the receiver is given by,

$$
\begin{gathered}
I=\hat{a}^{\prime} a^{\prime}=\left[\begin{array}{ll}
\left(e^{-i \delta}-1\right) \sin \beta \cos \beta & 0
\end{array}\right]\left[\begin{array}{c}
\left(e^{-i \delta}-1\right) \sin \beta \cos \beta \\
0
\end{array}\right] \\
=\left[2-\left(e^{i \delta}+e^{-i \delta}\right)\right] \sin ^{2} \beta \cos ^{2} \beta=\sin ^{2} \frac{\delta}{2} \sin ^{2} 2 \beta
\end{gathered}
$$

From the above equation, we obtain $\mathrm{I}=0$ when

$$
\beta=0, \frac{\pi}{2}, \ldots, n \frac{\pi}{2} \quad \text { or } \quad \delta=0,2 \pi, \ldots, n 2 \pi
$$


The locus of the retardation direction at different points on the specimen (at $\beta=$ constant values) are plotted which is known as isoclinic values. Similarly, the retardation values are calculated (where $\delta=2 n \pi$ ) known as isochromatic which become coloured when white light is used instead of monochromatic light. Hence, plane polariscope provides the results superposed by isochromatic and isoclinic values on a birefringent specimen. Thus, we can analyse the stress based on photoelastic method known as the classical method of photoelasticity.

\subsubsection{Circular polariscope}

Two additional quarter wave plates are introduced between two polarizers whose fast axes make an angle of $\pm 45^{0}$ with the polarizer and analyser respectively to obtain the circular polariscope. Two quarter-wave plate's fast axis should be arranged in crossed type so that no light can pass through the analyser thus obtaining dark field circular polariscope. To obtain light field circular polariscope, fast axes of quarter-wave plates should be made parallel to each other.

The setup of circular polariscope as shown in Figure 2.4 includes optical elements provided below,

I) A first polarizer (linear) $\mathrm{P}_{90}$ with the vertical optical axis.

II) First quarter wave plate oriented at $45^{0}$ with respect to $\mathrm{O}$ (object reference) $\mathrm{x}$-axis.

III) Specimen (birefringent material) $\boldsymbol{R}_{\boldsymbol{\beta}}(\delta)$ with retardation $\delta$, where fast axis is oriented at $\beta$ angle with respect to $\mathrm{O} x$ axis. 
IV) Second quarter-wave plate $\left(\mathrm{Q}_{ \pm 45}\right)$ is crossly arranged at an angle of $45^{0}$ with respect to $\mathrm{O} x$-axis (crossly arranged dark field) or $+45^{0}$ with respect to $\mathrm{O} x$-axis (parallelly arranged light field)

V) Second polarizer (linear) $\mathbf{P}_{0}$ with the horizontal optical axis.

Here, we compute the optical transformation of light rays in circular polariscope by the aid of Jones calculus.

\section{Mathematical modelling of polariscope on Jones calculus}

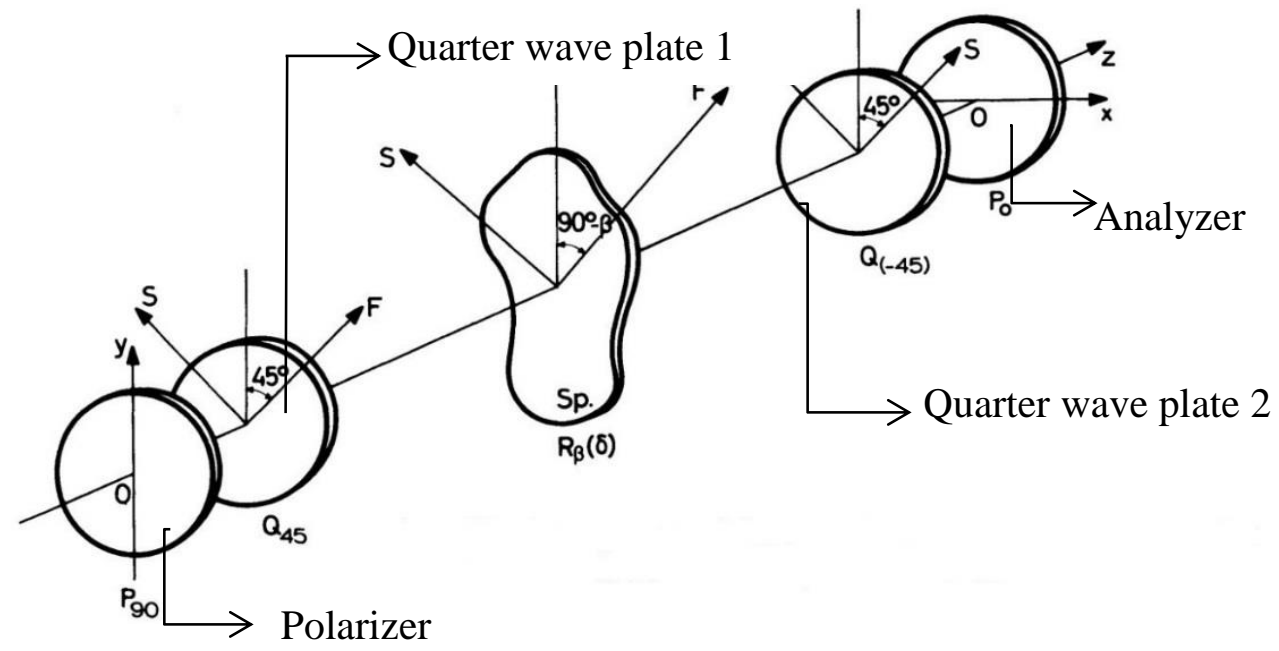

Figure 2.4: Arrangement of optical elements in circular polariscope

We compute optical transformation by cross polarizers at $90^{\circ}$ and quarter wave plates arranged at $\pm 45^{\circ}$ with the $\mathrm{O} x$-axis. Thus, $\delta=90^{\circ}$ and $\beta= \pm 45^{\circ}$ for the two polarizers and two quarter wave plates are,

$$
\begin{gathered}
\mathrm{Q}_{45}=\frac{1}{2}\left[\begin{array}{cc}
i+1 & i-1 \\
i-1 & i+1
\end{array}\right]=\frac{i+1}{2}\left[\begin{array}{cc}
1 & i \\
i & 1
\end{array}\right], \\
\mathrm{Q}_{-45}=\frac{1}{2}\left[\begin{array}{cc}
i+1 & -(i-1) \\
-(i-1) & i+1
\end{array}\right]=\frac{i+1}{2}\left[\begin{array}{cc}
1 & -i \\
-i & 1
\end{array}\right],
\end{gathered}
$$


On computation via Jones calculus, a' that leaves from the analyser on dark field circular polariscope is given by,

$$
a^{\prime}=P_{0} Q_{-45} R_{\beta}(\delta) Q_{45} a
$$

By combining equations, we get,

$$
\mathbf{a}=\left[\begin{array}{ll}
1 & 0 \\
0 & 0
\end{array}\right] \frac{i+1}{2}\left[\begin{array}{cc}
1 & -i \\
-i & 1
\end{array}\right]\left[\begin{array}{cc}
e^{i \delta} \cos ^{2} \beta+\sin ^{2} \beta & \left(e^{i \delta}-1\right) \sin \beta \cos \beta \\
\left(e^{i \delta}-1\right) \sin \beta \cos \beta & e^{i \delta} \sin ^{2} \beta+\cos { }^{2} \beta
\end{array}\right] \frac{i+1}{2}\left[\begin{array}{ll}
1 & i \\
i & 1
\end{array}\right]\left[\begin{array}{l}
0 \\
1
\end{array}\right]
$$

On matrix transformation, the final amplitude of intensity is given by,

$$
a^{\prime}=\frac{1}{2}\left[\begin{array}{c}
e^{i \delta}-1 \\
0
\end{array}\right]
$$

Thus, the resulting intensity will be,

$$
I=\widetilde{a}^{\prime} a^{\prime}=\frac{1}{4}\left[\begin{array}{ll}
e^{-i \delta}-1 & 0
\end{array}\right]\left[\begin{array}{c}
e^{i \delta}-1 \\
0
\end{array}\right]=\sin ^{2} \frac{\delta}{2}
$$

Equation (2.23) implicates that for dark field circular polariscope, the isochromatic fringe of integer order is obtained.

In bright field circular polariscope, the Jones vector of amplitude a' for emerging light is given by,

$$
a^{\prime}=P_{0} Q_{45} R_{\beta}(\delta) Q_{45} a
$$

or

$$
\mathbf{a}=\left[\begin{array}{ll}
1 & 0 \\
0 & 0
\end{array}\right] \frac{i+1}{2}\left[\begin{array}{ll}
1 & i \\
i & 1
\end{array}\right]\left[\begin{array}{ll}
e^{i \delta} \cos ^{2} \beta+\sin ^{2} \beta & \left(e^{i \delta}-1\right) \sin \beta \cos \beta \\
\left(e^{i \delta}-1\right) \sin \beta \cos \beta & e^{i \delta} \sin ^{2} \beta+\cos ^{2} \beta
\end{array}\right] \frac{i+1}{2}\left[\begin{array}{ll}
1 & i \\
i & 1
\end{array}\right]\left[\begin{array}{l}
0 \\
1
\end{array}\right]
$$


On matrix transformation, the final amplitude is given by

$$
a^{\prime}=\frac{1}{2}\left[\begin{array}{c}
e^{i \delta}+1 \\
0
\end{array}\right]
$$

Thus, the resulting intensity will be,

$$
I=\widetilde{a}^{\prime} a^{\prime}=\frac{1}{4}\left[\begin{array}{ll}
e^{-i \delta}+1 & 0
\end{array}\right]\left[\begin{array}{c}
e^{i \delta}+1 \\
0
\end{array}\right]=\cos ^{2} \frac{\delta}{2}
$$

Half-integer order isochromatic is obtained in bright field circular polariscope.

\subsubsection{Photoelastic modulator}

Photoelastic modulator (PEM) principle is based on the photoelastic phenomenon of the quartz, calcite and various other photoelastic specimens. The solid sample is fully transparent which is birefringent upon compression or stretching. So, when light passes through the photoelastic specimen, it attains different speed and polarisation direction. The operation principle of PEM is based on the photoelastic principle [51] where birefringence is induced on the mechanically stressed sample. These are resonant material producing oscillating birefringence of certain frequency within the range of ultrasound waves $(20 \mathrm{kHz}$ to $100 \mathrm{kHz})$.

PEM comprises of transparent thin rectangular bar (fused silica) fixed with a piezoelectric transducer. The bar oscillates axially which is dependent on the length of the bar attached to the piezoelectric transducer. The amplitude is modulated and tuned to the frequency of the bar by the designed electronic circuit. The birefringence is maximum at the centre of the fused silica bar. 
On compression of optical element i.e. rectangular bar, polarisation component parallel to modulator axis propagates faster compared to the transverse polarisation component. The vertical component lags the horizontal component during compression and the opposite happens during stretching or vice versa.

\subsubsection{Retardation effects of compression and extension}

The effect of the modulator on a monochromatic light wave depends on the plane of polarisation. The plane of polarisation is at $45^{\circ}$ to the modulator axis before passing through the modulator. If the optical element is relaxed, the polarisation of light will remain unaltered. On perturbation with the optical element, the polarisation of transmitted light will be altered in different direction depending upon material property. Thus, the light will propagate at different component at different orientation on interrogation with optical element. The phase difference between the components of optical element at instantaneous period is coined as retardation. The peak retardation is the amplitude of the sinusoidal retardation which is a function of time. It is mentioned in terms of length (units) and is given by,

$$
A(t)=z\left[n_{x}(t)-n_{y}(t)\right]
$$

Here $\mathrm{n}_{\mathrm{x}}(\mathrm{t})$ and $\mathrm{n}_{\mathrm{y}}(\mathrm{t})$ are instantaneous refractive index values along $\mathrm{x}$ and $\mathrm{y}$-axis. General units of retardation are in terms of length (nanometres, microns), waves (quarter-wave, half-wave), and phase angle (radians, degrees). It can be expressed as,

$$
R(\text { nanometers })=\frac{2 \pi R(\text { nanometers })}{\lambda(\text { nanometer })}
$$


Or,

$$
R(\text { degrees })=\frac{360 R(\text { nanometers })}{\lambda(\text { nanometers })}
$$

\subsubsection{Retardation measurement by PEM}

The optical setup employed for measuring the retardation of the birefringent sample is shown in Figure 2.5. The vertical setup is convenient and unlikely vibration is absorbed by the Sorbothane dampers.

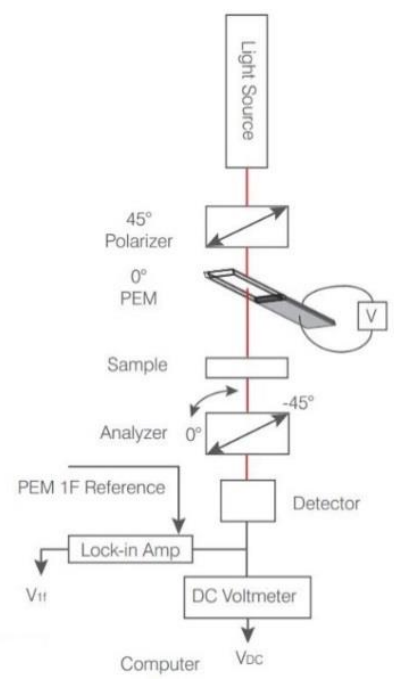

Figure 2.5: Arrangement of optical elements on photoelastic modulation principle

To avoid collimating and focusing problem, the laser has been implemented as a light source. The polarizer is just kept beneath light source oriented at $+45^{0}$ with respect to the PEM retardation axis. Reference retardation axis is defined at $0^{0}$ coordinate systems of the setup. The modified light is passed through a sample and then to the detector. Applying low pass filter before voltmeter removes unwanted noise and overshoot in the signals. The optimum values for PEM retardation should be set to $\mathrm{R}=0.38$ waves $=2.40$ radians. Thus, two signals are recorded. 
1) Lock- in amplifier identifies the RMS voltage $V_{1 f}$ at $1 \mathrm{f}$ frequency signal.

2) Average voltage or $V_{D C}$ signals recorded by a digital voltmeter.

The ratio value of lock-in amplifier and average voltage is computed which is independent of variation in light intensity and transmission.

$$
R_{1 f}=\frac{V_{1 f}}{V_{D C}}
$$

The output values are analysed by using Mueller calculus. Recorded signal amplitude are instantaneous values known as peak voltage amplitudes. For average amplitude values, we find the RMS values of $\sqrt{2}$ which proves that,

$$
\sqrt{2} R_{1 f}=2 J_{1}(A) \sin \delta
$$

Here $\delta$ is the sample retardation angle values (e.g. radians), $J_{1}$ (A) is Bessel function amplitude and $\mathrm{R}_{1 \mathrm{f}}$ is the ratio of lock-in amplifier signal to voltmeter signal values.

Hence,

$$
\begin{aligned}
& \sin \delta=\frac{R_{1 f}}{\sqrt{2} J_{1}(A)} \\
& \delta=\sin ^{-1}\left[\frac{R_{1 f}}{\sqrt{2} J_{1}(A)}\right]
\end{aligned}
$$




\subsubsection{Retardation magnitude and fast axis orientation calculation}

There are two techniques of calculating retardation magnitude and fast axis direction.

The sample is kept on a rotation stage. The sample is rotated on the rotation stage until the ratio $R_{1 f}$ is maximum. The magnitude of retardation is given by equation (2.35). The sample is kept in reference axis with PEM retardation axis.

Sample at fixed orientation

a. Calculate the retardation measurement using setup above noted as (second polarizer at $\left.-45^{0}\right) \delta_{1}$.

b. The second polarizer is rotated to $0^{0}$ and noted as $\delta_{\text {III }}$.

c. Thus retardation magnitude can be calculated as,

$$
\delta=\sqrt{\delta_{1}^{2}+\delta_{I I}^{2}}
$$

d. Thus, fast axis can be calculated as

$$
\rho=\frac{1}{2} \tan ^{-1} \frac{\delta_{I I}}{\delta_{I}}
$$

The system serves the rapid full-field measurement of retardation magnitude and fast axis direction. It utilises beam splitter and two polarizer/detector assemblies. The system is used for measurement of small values of retardation $\delta$ (eg. $\delta<<\pi / 2$ ). For values, less than this the accuracy and precision become poor, limiting resolution in terms of micrometre. PEM approach has been implemented by HINDS system to measure birefringence in the specimen. 


\subsection{Mathematical modelling of birefringent effects in plastic optics}

Birefringence modelling of plastic optics is performed to inspect its effects on image formation and degradation. Effect of stress birefringence is critical for the polarisation[52-54] sensitive optical systems like optical lithography[55, 56], lenses, LCD (Liquid Crystal Display) projectors and various other plastic optics. High birefringence on the plastic substrates reduces storage density[57] on the magneto-optical data storage field. Stress birefringence in the LCD projector component alters the colour gamut in the projected range. The difference in coefficients of thermal expansion, temperature gradient, pressure inertial and vibratory loads during manufacturing induces birefringence in the material. Induced birefringence results in wavefront [58, 59] error and polarisation aberration $[60,61]$ through the optical system. Birefringence modelling is carried out in an anisotropic medium by using Maxwell's equations [62]. Dielectric impermeability tensor of the material is represented by $\mathrm{C}_{\mathrm{ij}}$ which is the modulus value of second-order tensor and direction given by,

$$
C_{i j}=\left[\begin{array}{lll}
C_{11} & C_{12} & C_{13} \\
C_{21} & C_{22} & C_{23} \\
C_{31} & C_{32} & C_{33}
\end{array}\right]
$$

The dielectric impermeability tensor on the specimen is expressed by,

$$
\sum_{i j}^{n} C_{i j} x_{i}=1 \rightarrow C_{11} x_{1}^{2}+C_{22} x_{2}^{2}+C_{33} x_{3}^{2}+2 C_{12} x_{1} x_{2}+2 C_{23} x_{2} x_{3}+2 C_{13} x_{1} x_{3}=1
$$

The above equation represents the second-degree surface where $C_{i j}$ represents the coefficient values of the surface. The dielectric impermeability stress tensor values are the intrinsic photoelastic constant of the material. 
On applying mechanical stress on the material, optical properties are modified due to alteration on dielectric impermeability tensor. Refractive index modification is due to imparted stress that modifies the impermeability tensor which alters the size, shape and orientation of the material. Stress dielectric impermeability tensor of material due to mechanical stress is given by,

$$
\Delta C_{i j}=C_{i j}-C_{i j}^{o}=q_{i j k l} \sigma_{k l}
$$

where $\mathrm{q}$ is the stress-optic coefficient matrix and $\sigma$ is the stress tensor.

\subsubsection{Numerical Analysis}
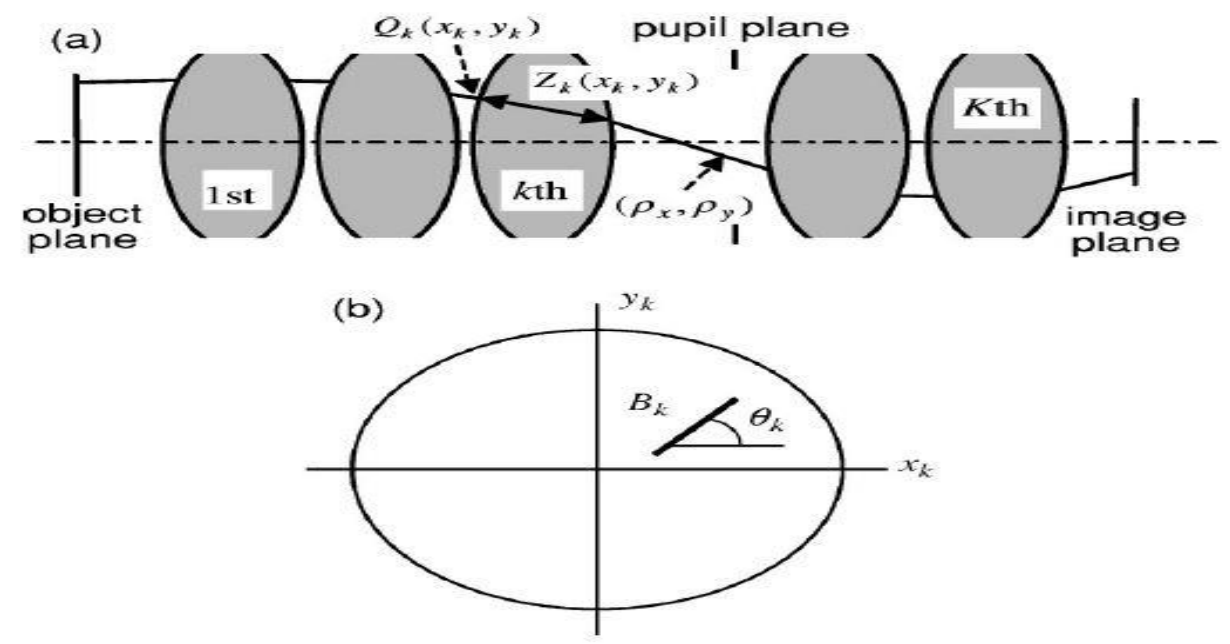

Figure 2.6: Projection lens composed of K birefringent elements[63]

The arrangement of the projection lens is shown in Figure 2.6 is composed of total Kelements. Each optical element is represented by $\mathrm{k}^{\text {th }}$ element $(1<\mathrm{k}<\mathrm{K})$ with their birefringence magnitude $B_{k}\left(x_{k}, y_{k}\right)$ and its fast axis direction $\theta_{k}\left(x_{k}, y_{k}\right)$ as a function of positional ordinate[15]. The deviation in wavefront of a beam is represented by $Q_{k}\left(x_{k}, y_{k}\right)$ which is the product of magnitude of birefringence 
$B_{k}\left(x_{k}, y_{k}\right)$ and $Z_{k}\left(x_{k}, y_{k}\right)$, where $Z_{k}\left(x_{k}, y_{k}\right)$ is the path length. The birefringence values $B_{k}\left(x_{k}, y_{k}\right)$ are represented in $\mathrm{nm} / \mathrm{cm}$ whereas path length $Z_{k}\left(x_{k}, y_{k}\right)$ in $\mathrm{cm}$ units, resultant wavefront deviation is given by,

$$
Q_{k}\left(x_{k}, y_{k}\right)=\frac{2 \pi}{\lambda} B_{k}\left(x_{k}, y_{k}\right) Z_{k}\left(x_{k}, y_{k}\right)
$$

For partial, random or incoherent polarised light we can use Mueller matrices [64]. Considering the fully polarised light, the state of polarisation $Q_{k}\left(x_{k}, y_{k}\right)$ for received intensity is represented by a Jones vector[65] $\left(p_{x, k-1}, p_{y, k-1}\right)$ after leaving the $k^{\text {th }}$ element is calculated by,

$$
\left(\begin{array}{c}
p_{x, k} \\
p_{y, k}
\end{array}\right)=M_{k}\left(x_{k}, y_{k}\right)\left(\begin{array}{c}
p_{x, k-1} \\
p_{y, k-1}
\end{array}\right)
$$

Where the Jones matrix $M_{k}\left(x_{k}, y_{k}\right)$ is given by,

$$
M_{k}\left(x_{k}, y_{k}\right) \equiv R\left(-\Theta_{k}\right)\left[\begin{array}{cc}
e^{-i \Phi k / 2} & 0 \\
0 & e^{-i \Phi k / 2}
\end{array}\right] \mathrm{R}\left(\Theta_{k}\right)
$$

with

$$
R\left(\theta_{k}\right)=\left[\begin{array}{rr}
\cos \theta_{k} & \sin \theta_{k} \\
-\sin \theta_{k} & \cos \theta_{k}
\end{array}\right]
$$

Hence on applying polarisation state for all elements, the final distribution of polarisation state is given by,

$$
\left[\begin{array}{c}
p_{x}\left(p_{x}, p_{y}\right) \\
p_{y}\left(p_{x}, p_{y}\right)
\end{array}\right]=\Pi_{k=K,-1}^{1} \quad M_{k}\left(x_{k}, y_{k}\right)\left(\begin{array}{c}
\cos \omega \\
\sin \omega
\end{array}\right)
$$


Jones matrix elements represent the polarization components by $m_{\alpha \beta}\left(p_{x}, p_{y}\right)(\alpha$, $\beta=1,2)$ which clearly represents its pupil coordinate dependence by,

$$
\begin{aligned}
& p_{x}\left(p_{x}, p_{y}\right)=m_{11}\left(p_{x}, p_{y}\right) \cos \omega+m_{12}\left(p_{x}, p_{y}\right) \sin \omega \\
& p_{y}\left(p_{x}, p_{y}\right)=m_{21}\left(p_{x}, p_{y}\right) \cos \omega+m_{22}\left(p_{x}, p_{y}\right) \sin \omega
\end{aligned}
$$

Above equation represents the pupil transmission characteristics affected by polarisation aberrations. The spectrum distribution obtained on the pupil plane $\hat{\mathrm{O}}\left(p_{x}^{\prime \prime}, p_{y}^{\prime \prime}\right)$ when an object rays $\mathrm{O}(x, y)$ illuminates material of orthogonal polarizations $\left(p_{x}^{\prime \prime} \equiv p_{x}+p_{x}^{\prime}\right.$ and $\left.p_{y}^{\prime \prime} \equiv p_{y}+p_{y}^{\prime}\right)$, is calculated by,

$$
\hat{\mathrm{O}}\left(p_{x}^{\prime \prime}, p_{y}^{\prime \prime}\right)=\iint O(x, y) e\left[-i 2 \pi\left(p_{x}^{\prime \prime} x+p_{y}^{\prime \prime} y\right) d x d y\right. \text {, }
$$

which is the Fourier transformation of $\mathrm{O}(x, y)$, where $p_{x}^{\prime \prime} \equiv p_{x}+p_{x}^{\prime}$ and $p_{y}^{\prime \prime} \equiv$ $p_{y}+p_{y}^{\prime}$ are introduced to simplify the integration equation. The Fourier transformation of the above equation is computed to obtain the image amplitude as per the Abbe theory of image formation on $p_{\gamma}\left(p_{x}, p_{y}\right)(\gamma=x, y)$ which is given by,

$U_{\gamma}(x, y)=\iint \hat{\mathrm{O}}\left(p_{x}^{\prime \prime}, p_{y}^{\prime \prime}\right) p_{\gamma}\left(p_{x}, p_{y}\right) \times e\left[-i 2 \pi\left(p_{x} x+p_{y} y\right)\right] d p_{x} d p_{y}$

Wavefront aberration excluding birefringence is zero, integration is carried out within $\left(p_{x}^{2}+p_{y}^{2} \leq 1\right)$. Normalisation of positional coordinate is obtained by multiplying $\lambda / N A$. Thus, image intensity distribution is obtained as,

$$
\hat{I}_{w}(x, y)=\left|U_{x}(x, y)\right|^{2}+\left|U_{y}(x, y)\right|^{2}
$$


Considering the different polarisation state represented by $p_{x}\left(p_{x}, p_{y}\right)$ and $p_{y}\left(p_{x}, p_{y}\right)$ and small numerical aperture, there won't be any interference thus less chance of inclusive errors. To eliminate the polarisation effect of an illumination beam, we integrate equation with respect to $\omega$,

$$
\begin{aligned}
\hat{I}(x, y) & =\int_{0}^{\pi} \hat{I} \omega(x, y) d \omega / \int_{0}^{\pi} d \omega \\
& =\frac{1}{2} \sum_{\alpha=1}^{2} \sum_{\beta=1}^{2}\left|u_{\alpha \beta}(x, y)\right|^{2},
\end{aligned}
$$

where

$u_{\alpha \beta}(x, y)=\iint \hat{\mathrm{O}}\left(p_{x}^{\prime \prime}, p_{y}^{\prime \prime}\right) m_{\alpha \beta}\left(p_{x}, p_{y}\right) \times e\left[-i 2 \pi\left(p_{x} x+p_{y} y\right)\right] d p_{x} d p_{y}$

Integrating the above equation with its respective source coordinate, the intensity of the image is obtained. On integration under partial coherent condition, resultant illumination intensity is obtained as,

$$
\begin{aligned}
I(x, y) & =\iint_{\Sigma} \widehat{I}(x, y) d p_{x}^{\prime} d p_{y}^{\prime} / \iint_{\Sigma} d p_{x}^{\prime} d p_{y}^{\prime} \\
& =\frac{1}{2} \sum_{\alpha=1}^{2} \sum_{\beta=1}^{2}\left(\iint\left|u_{\alpha \beta}(x, y)\right| 2 d p_{x}^{\prime} d p_{y}^{\prime} / \iint d p_{x}^{\prime} d p_{y}^{\prime}\right)
\end{aligned}
$$

The integration range $\Sigma$ is given by $p_{x}^{\prime 2}+p_{y}^{\prime 2} \leq \epsilon^{2}$ with $\epsilon$ denoting the degree of coherence, where $\epsilon=0$ is coherent whereas $\epsilon=1$ is incoherent.

Hence,

$$
\mathrm{I}(\mathrm{x}, \mathrm{y})=\frac{1}{2} \sum_{\alpha=1}^{2} \sum_{\beta=1}^{2} I_{\alpha \beta}(x, y)
$$

with,

$$
I_{\alpha \beta}(x, y) \equiv \iint_{\Sigma}\left|u_{\alpha \beta}(x, y)\right|^{2} d p_{x}^{\prime} d p_{y}^{\prime} / \iint_{\Sigma} d p_{x}^{\prime} d p_{y}^{\prime}
$$


The obtained image intensity value matches with that of pupil function $\mathrm{m}_{\alpha \beta}\left(p_{x}, p_{y}\right)$. Since equation $(2.52)$ is the intensity for the unpolarised beam, it is possible to give the same equation by $I_{\alpha \beta}(x, y)$ representing polarisation state. $I_{11}(x, y)$ and $I_{22}(x, y)$ represents polarisation component in the $\mathrm{x}$ and $\mathrm{y}$ direction whereas $I_{21}(x, y)$ and $I_{12}(x, y)$ represents polarisation component influenced by birefringence in $\mathrm{x}$ and $\mathrm{y}$ direction. Thus, normalising the Jones matrix for easy computation we get,

$$
\left|m_{11}\left(p_{x}, p_{y}\right)\right|^{2}+\left|m_{12}\left(p_{x}, p_{y}\right)\right|^{2}=1
$$

Where,

$$
m_{21}\left(p_{x}, p_{y}\right)=-m_{12}^{*}\left(p_{x}, p_{y}\right), m_{22}\left(p_{x}, p_{y}\right)=m_{11}^{*}\left(p_{x}, p_{y}\right)
$$

The complex conjugate component is represented by an asterisk for the corresponding image component. For symmetrical objects, $O *(x, y)= \pm O(-$ $x,-y)$, the mapped image components will have symmetry of $I_{11}(x, y)=I_{22}(-x,-y)$ and $I_{12}(x, y)=I_{21}(-x,-y)$, which results $I(x, y)=I(-x,-y)$. Hence, cause of image degradation is due to following reasons,

Formation of weak image intensity values i.e. $I_{12}(x, y)$ and $I_{21}(x, y)$.

Deterioration in $I_{11}(x, y)$ and $I_{22}(x, y)$ because of aberrations components $m_{11}\left(p_{x}, p_{y}\right)$ and $m_{22}\left(p_{x}, p_{y}\right)$ respectively.

Slight displacement in image intensity values (distortion) from their optical axis at $\mathrm{I}_{11}(x, y)$ and $I_{22}(x, y)$ occurs although they are symmetrical with respect to $(x, y, z)=(0,0,0)$. 


\subsection{Effects of Birefringence in Imaging in plastic optics}

During manufacturing of photolithographic lenses, the feature size is demanded to be precise and accurate but there is an alteration in feature size due to birefringence. It is induced birefringence due to non-uniform temperature distribution and stress released during manufacturing [66]. As photoelastic materials are birefringent, they behave as temporary wave plates refracting light with varying amplitude and orientation relying on stress state of the material. The effect of birefringence in imaging is well verified by the conducted experimental research in section 2.9. The degradation of the image (contrast) is squarely proportional to the birefringence magnitude. On highly birefringent materials, the axis distribution is randomly distributed. From the experiment conducted by Suzuki and Unno[63], it concludes that in the higher birefringent material there is a randomly distributed fast axis distribution present in the material which in turn degrades the image contrast as illustrated in Figure 2.7.

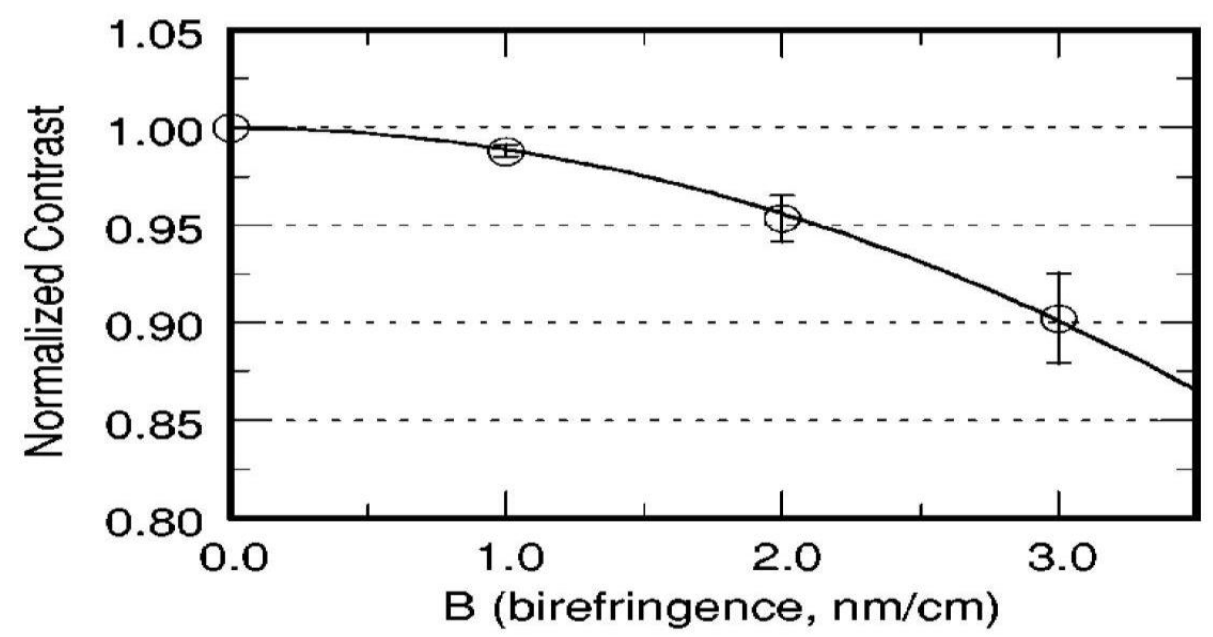

Figure 2.7: Relationship between $B$ (magnitude of birefringence) and normalized contrast[63] 


\subsection{Effects of birefringence in imaging (Experimental verification)}

Mathematically, we have formulated that birefringence decreases the contrast of the image. To verify this experimentally, we took three sample manufactured from the same mould at the same temperature and pressure to ensure the specimens are thoroughly uniform and identical. It is almost ideal to find a sample of uniform refractive index. Then, we gradually stacked three identical sample above the subject continuously and measured its contrast as well. The subject is a black and white fringe of $1.5 \mathrm{~mm}$ width gap. The contrast in the sample decreases on gradually piling up the birefringent samples. The subject consists of ten black and white patterns as shown in Figure 2.8. The subject is imaged using polariscope with stacks of birefringent PMMA sample and the contrast value is computed on the specified ROI (Region of Interest). The intensity values and contrast of the region of interest (ROI) of the reference image as in Figure 2.9 is calculated and compared with stacks of birefringent samples placed above it.
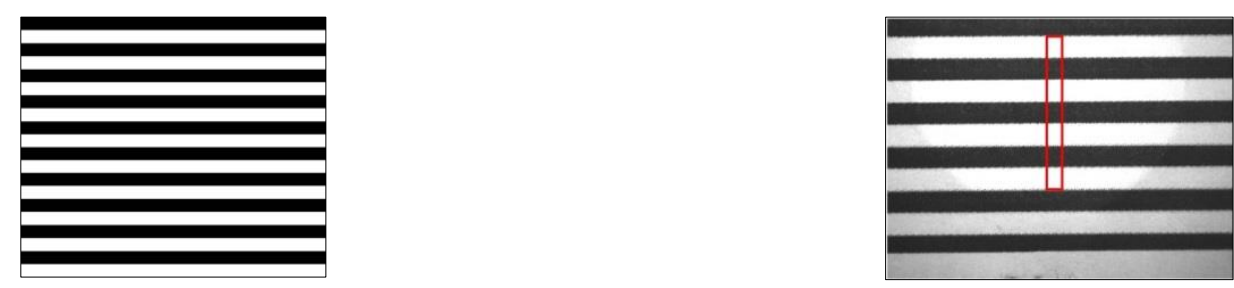

Figure 2.8: Left (Black and white pattern subject), right (Image of the subject)

Figure 2.10 represents the ROI of the reference sample image and for comparative analysis, the ROI of the stacked sample image is computed.

Figure 2.9: ROI (Region of Interest) of image of the subject pattern 


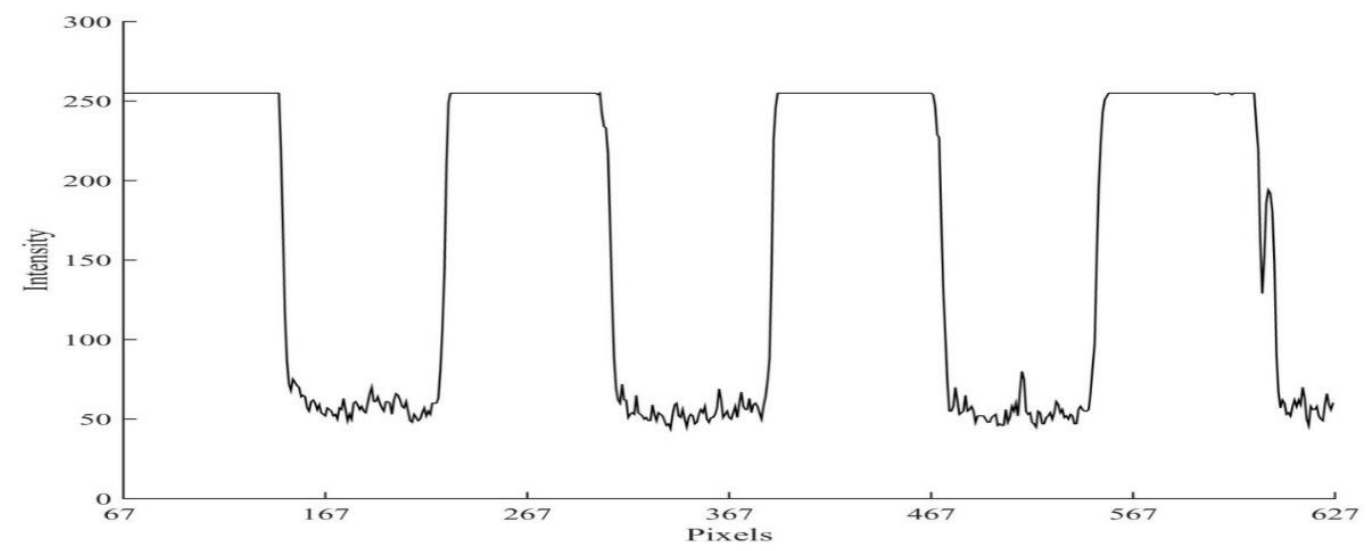

Figure 2.10: Intensity profile of ROI (Region of Interest) of image of the subject pattern

The intensity values of the ROI of the reference image is as shown in the Figure

2.11 for further analysis.
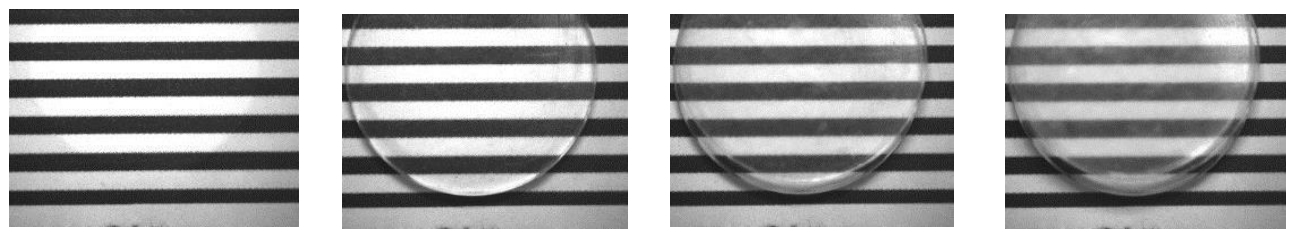

Figure 2.11: Reference image without sample and images on adding successive number of disks

The contrast values are calculated for the reference subject and on adding birefringent disk above the subject which is clearly represented in the Figure 2.11. The contrast of the subject is decreased by adding a number of birefringent disks which has been proved theoretically by Unno and Suzuki [15]. The experimental setup and calculation are performed to know the impact in imaging by measuring contrast with the addition of several birefringent disks. The calculated contrast in imaging on increasing birefringence by adding several birefringent disks are completely represented in the chart below. 
Normalised Contrast versus number of circular disk

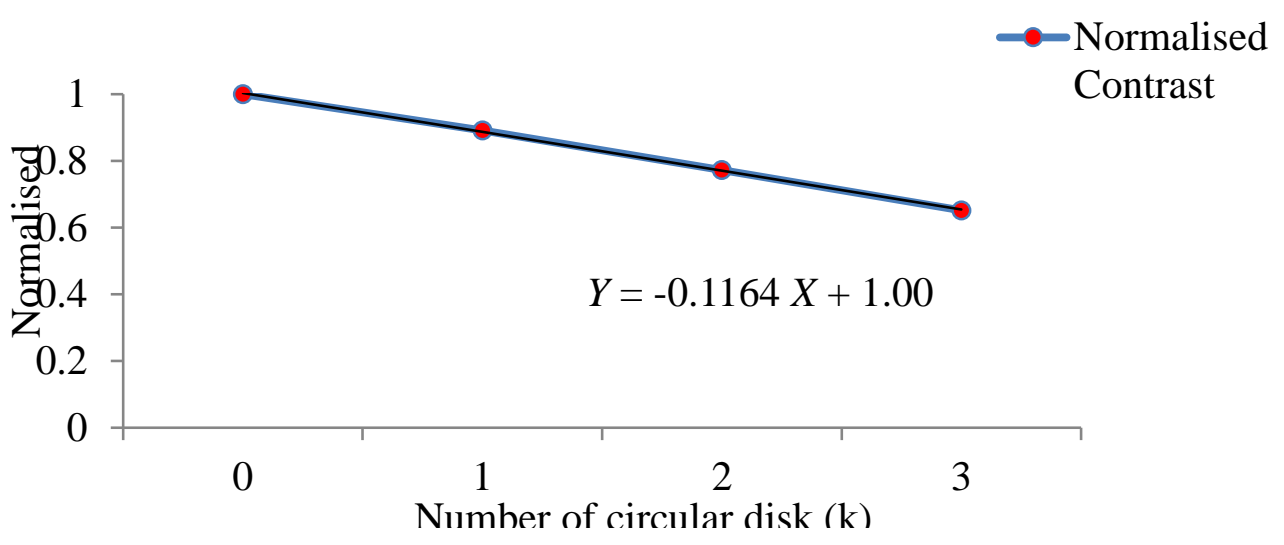

Figure 2.12: Relationship between contrast values versus number of circular disks The contrast of the image decreases on adding the number of birefringent disks which is evident from the Figure 2.12. In addition to that, the normalised contrast value is mapped to the increasing birefringence values on adding a birefringent sample. The Figure 2.13 indicates that contrast values decrease with an increase in birefringence values by adding birefringent disks.

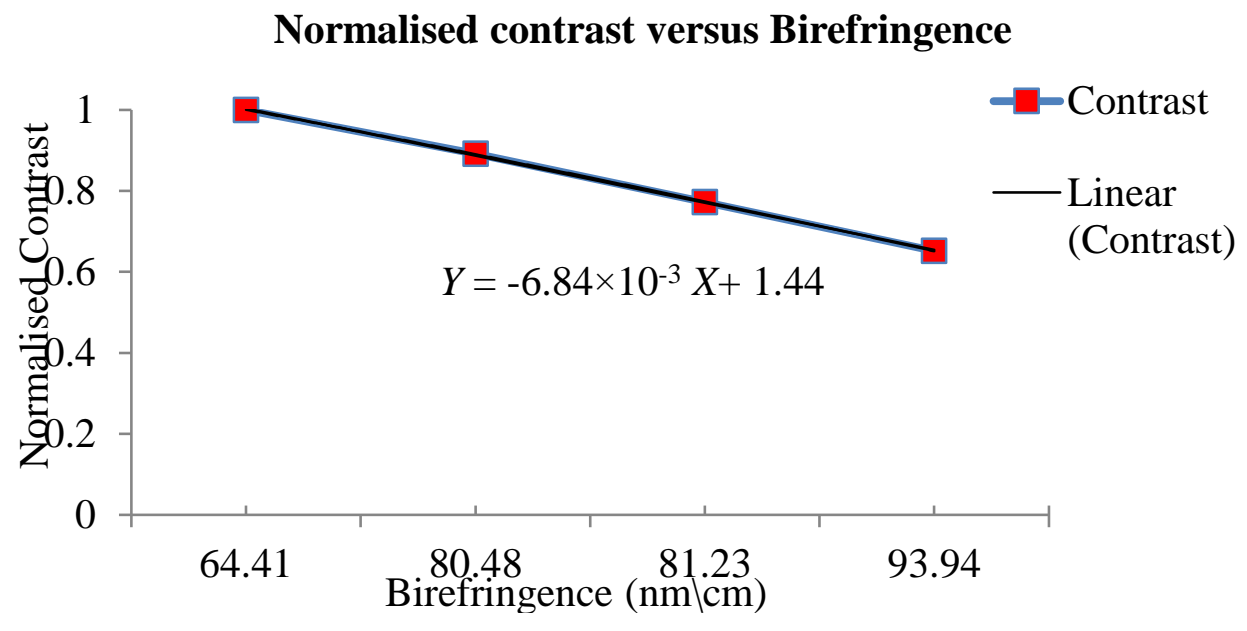

Figure 2.13: Relationship between contrast values versus increasing birefringence in the sample 


\subsection{Summary}

Various prevalent plastic optics are explored and analysed in detail. Their properties were classified based on different features particularly transmittance, refractive index, Abbe number and coefficient of thermal expansion. PMMA possess excellent transparency and material uniformity with moderate cost hence preferred among others. Once the material is selected, a manufacturing method for plastic optics is explored. Injection moulding is state of the art technology widely accepted for manufacturing various plastic optics. Recently 3D printing has also been involved in the production of plastic optics but has its own drawbacks. Plastic sample manufactured from 3D printing lacks the transparency and smoothness compared to injection moulding technique. The 3D printed samples were designed through $\mathrm{CAD}$ (computer aided drawing) modelling and implemented on CAM (Computer Aided Manufacturing) manufacturing via fused deposition modelling (FDM) method. Plastic optics are used in various applications like lighting, communication and security, healthcare, photovoltaic, sensors and military purpose.

Birefringence is a crucial issue in plastic optics for various applications. Variance on refractive index is also known as birefringence that degrades the material uniformity and strength. The temperature gradient, stress gradient, free mould shrinkage and various other factors cause birefringence in plastic optics. Birefringence highly affects plastic optics deterring the image contrast, defect and crack propagation in the material. It has a greater impact in biological imaging, lithography and various other applications. Mathematical modelling of 
birefringence in plastic optics has been computed and inferred that contrast and image distortion are critical effects due to birefringence.

Birefringence measurement systems were rigorously investigated. Two efficient polariscope techniques are proposed i.e. photoelastic modulator method and phase shift method. The photoelastic modulator is a point to point measurement system actuated by piezoelectric material limited by resolution and scanning speed. Phase shifting polariscope is one shot whole field resolution measurement system. Based on this principle, further research has been embarked for preparing a photoelastic system fully automated with an efficient algorithm and high computational speed. 


\section{Chapter 3: Birefringence modelling, simulation and}

\section{measurement}

The birefringent specimen is modelled in optical simulation software to observe its characteristic response in various wavelengths of light. We chose white light as a light source which passes through birefringent sample and the recorded images are thoroughly analysed. The optical software simulation lacks module for stressinduced birefringence in the sample specimen hence cannot simulate stressinduced birefringence. Thus, there is the essence of finite element modelling of plastic optics in CAD (Computer Aided Design) interface where applied stress is simulated to observe the birefringence characteristics. The simulated birefringence values are compared with the theoretical and experimental birefringence values and are in good agreement. Low birefringence polariscope is used to measure the stress-induced birefringence $[67,68]$ in the specimen. Here, stress-induced in birefringent specimen by two-point compression loading experiment is compared based on the theory of solid mechanics and finite element modelling (FEM).

\subsection{Introduction}

The object is of dimension 70 by 70 pixels with 24-bit depth. The entrance pupil is located at infinity, so chief rays in the object space are parallel to the optical axis. This file simulates placing a birefringent cube (calcite) on text and the resultant double image due to the ordinary and extraordinary indices of the material itself. The aspheric lens was optimised for this set of conjugates in sequential mode. 
The lens properties are based on Schott glass specifications at temperature of 20 degree Celsius and at one atmospheric pressure, the intended transmittance wavelength is 0.55 micrometres. The birefringent cube is of calcite material which has an ordinary refractive index of 1.66 and extraordinary refractive index of 1.48 . The detector material is made up of $\mathrm{Si}$ (Silicon) photoconductive material and has a resolution of $250 \times 250$ pixels. The size of the sensor is $20 \mathrm{~mm} \times 20 \mathrm{~mm}$ of peak irradiance $5.42 \times 10^{-01}$ watts $/ \mathrm{cm}^{2}$ with a total power of the detector approximately $2.56 \times 10^{-01}$ Watts. The radius of the lens in the simulation is of $99.20 \mathrm{~mm}$ with radial aperture $15 \mathrm{~mm}$ and the refractive index is of 1.52 . The opacity of the birefringent cube material is 70 percent which is composed of calcite material. The even aspherical lens of BK7 material is used for imaging purpose. The thickness of the slide, radial aperture of the slide, rectangular volume, aspherical lens and detector rectangle is of $1 \mathrm{~mm}, 12 \mathrm{~mm}, 15 \mathrm{~mm}, 10 \mathrm{~mm}$ and $10 \mathrm{~mm}$ respectively.

\subsection{Optical design and simulation via optical software}

\subsubsection{Design Specifications}

The parameters that have been employed in the optical design and simulation are presented in Table 3.1:

\begin{tabular}{|l|l|l|}
\hline Parameters & Units & Measurement \\
\hline Field of view (FOV) & $\mathrm{mm}^{2}$ & $17.6 \times 13.2$ \\
\hline Working distance (WD) & $\mathrm{mm}$ & 100 \\
\hline Back working distance (BWD) & $\mathrm{mm}$ & 210 \\
\hline Distortion & $\%$ & 0.05 \\
\hline Spectrum Range & $\mathrm{nm}$ & $486-656$ \\
\hline Total Length & $\mathrm{mm}$ & 310 \\
\hline
\end{tabular}

Table 3.1: Design specification of optical setup 


\subsubsection{Layout}

The layout gives the overall idea to the designer how the lenses are placed, spaced between each other to check

Whether there are any overlapping of optical elements.

If the rays are travelling on the assigned path.

If the rays are incident on the image plane correctly.

Moreover, it provides the overall axial length of the entire system of $310 \mathrm{~mm}$ as illustrated in Figure 3.1 with its working and back working distance of $100 \mathrm{~mm}$ and $210 \mathrm{~mm}$ respectively.

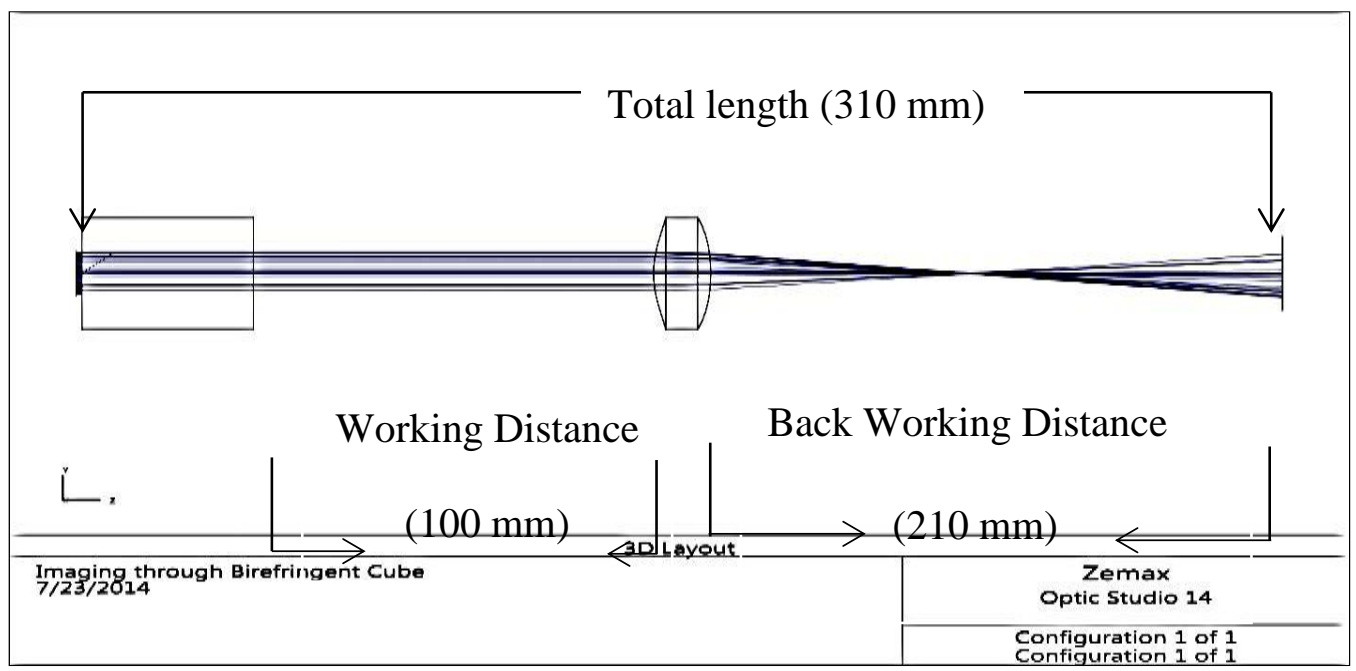

Figure 3.1: Two-dimensional layout of optical setup 


\subsubsection{Design data}

\begin{tabular}{|c|c|c|c|c|c|c|c|c|c|c|c|c|c|c|c|}
\hline & Object Type & Comment & Ref Object & Inside of & XPosition & Y Position & ZPosition & Tilt About X & Tilt About Y & Tilt About Z & Material & Radial Apertu & Thickness & Par 3(unused & ar 4(unused \\
\hline 1 & Source Rectangle - & & 0 & c & 0.000 & 0.000 & -1000 & 0.000 & 0.000 & 0.000 & & 50 & 1000000 & 1000 & 0 \\
\hline 2 & Slide. & LETTERFBMP & 0 & c & 0.000 & 0.000 & 0.000 & 0.000 & 0.000 & 0.000 & & 12.000 & 1000 & & \\
\hline $3^{3}$ & Rectangular Volume & & 0 & c & 0.000 & 0.000 & $1.000 E-003$ & 0.000 & 0.000 & 0.000 & CALCTE & 15.000 & 15.000 & 30.000 & 15.000 \\
\hline 4 & Even Asphere Lens & & 0 & c & 0.000 & 0.000 & 100.000 & 0.000 & 0.000 & 0.000 & $B K 7$ & 15.000 & 10.000 & & \\
\hline tr & Detector Rectangle - & & 0 & c & 0.000 & 0.000 & 210.000 & 0.000 & 0.000 & 0.000 & ABSORB & 10,000 & 10.000 & 250 & 250 \\
\hline
\end{tabular}

Table 3.2: Design specification deployed in optical setup

The parameters employed in the design and their respective separation is explicitly represented in Table 3.2 with their predetermined thickness, separation and aperture value.

\subsubsection{Wavelength}

The spectral range for the entire birefringence simulation is $486-656 \mathrm{~nm}$ as specified in Table 3.3.

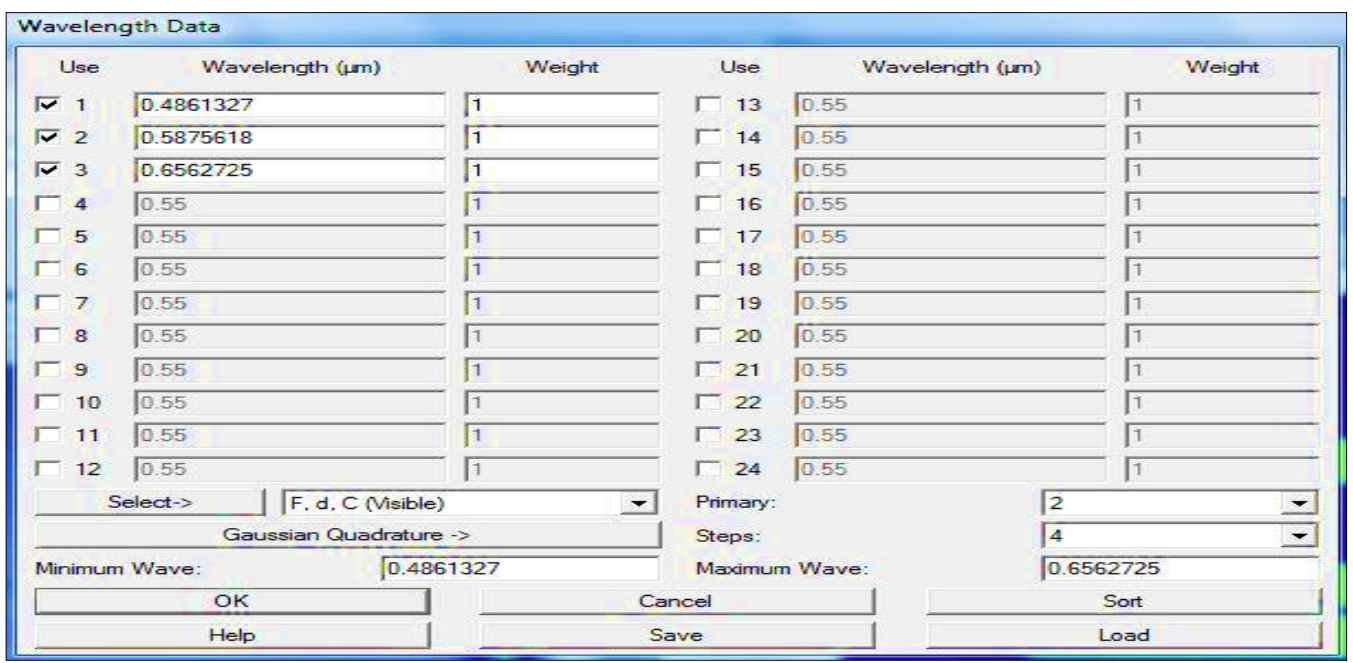

Table 3.3: Wavelength employed in the optical simulation setup 


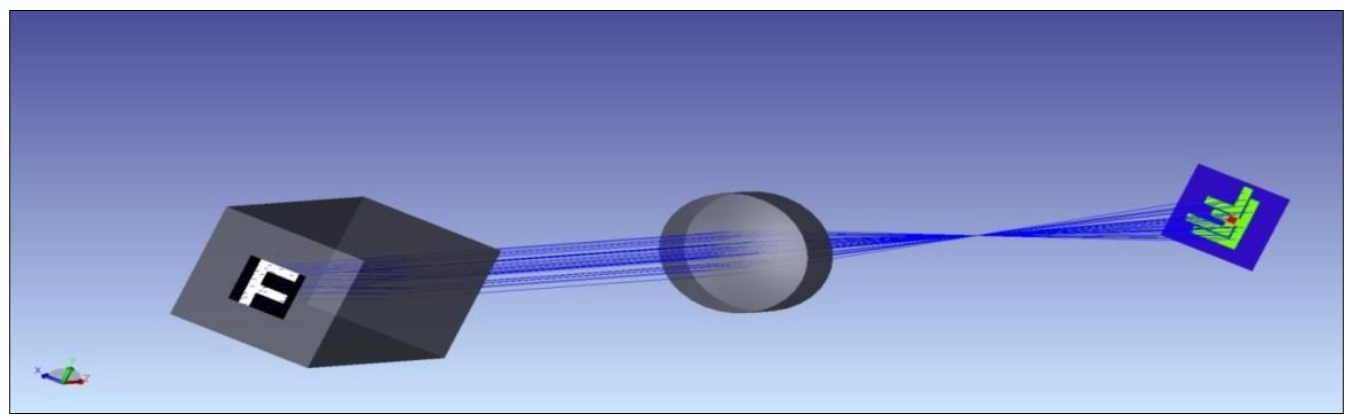

Figure 3.2: Three-dimensional layout of the optical setup employed in the simulation

Three-dimensional view of the setup with the birefringent cube, lens and the detector is represented in Figure 3.2.

\subsubsection{Detector}

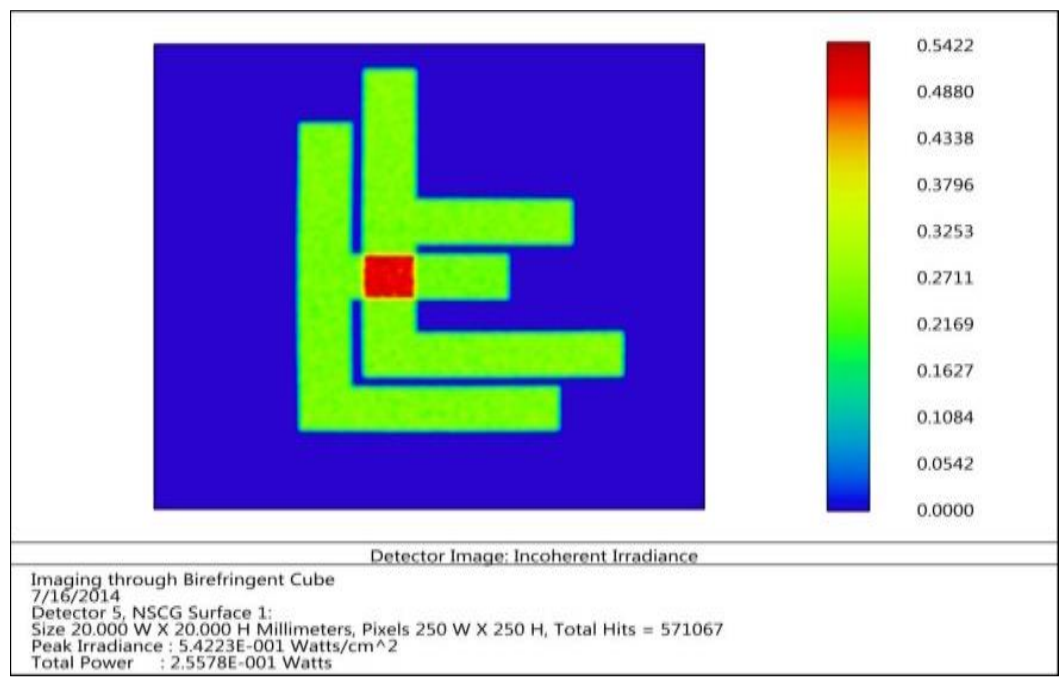

Figure 3.3: Image rendered in the detector on passing subject through birefringent cube

The detector is of size $20 \mathrm{~cm} \times 20 \mathrm{~cm}$ with $250 \times 250$ pixels resolution. As shown in Figure 3.3, the peak irradiance of the detector is $5.42 \times 10^{-1}$ Watts $/ \mathrm{cm}^{2}$ while the total power of the detector is $2.56 \times 10^{-1}$ Watts. 


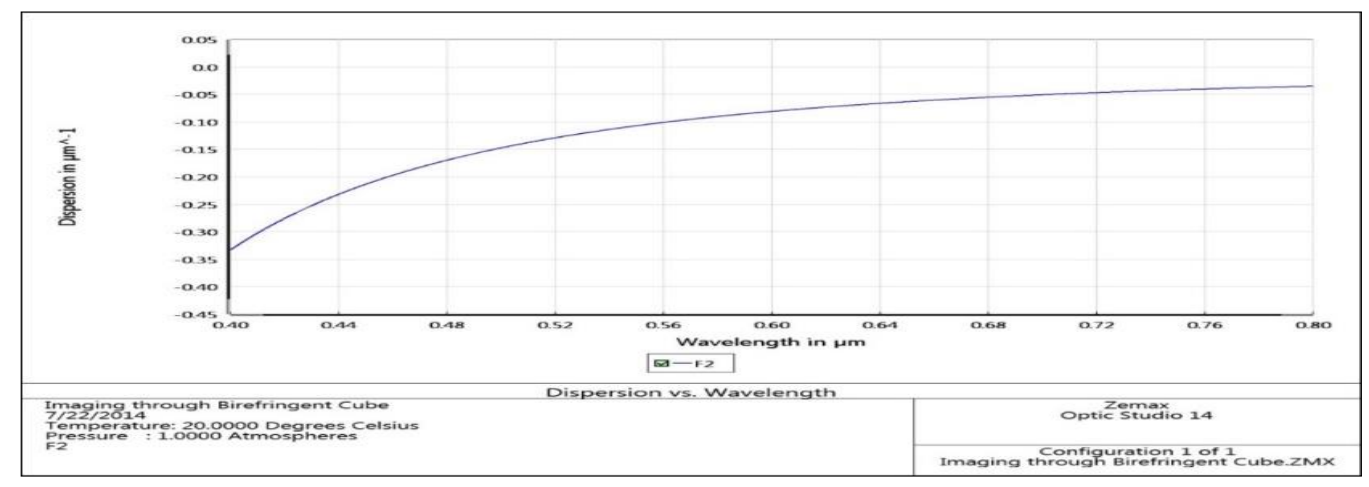

Figure 3.4: Dispersion characteristic curve with respect to wavelength variation

Dispersion of light is inversely proportional to the square of the wavelength as shown in Figure 3.4; hence for a light of shorter wavelength, there is a larger dispersion, vice versa for the longer wavelength.

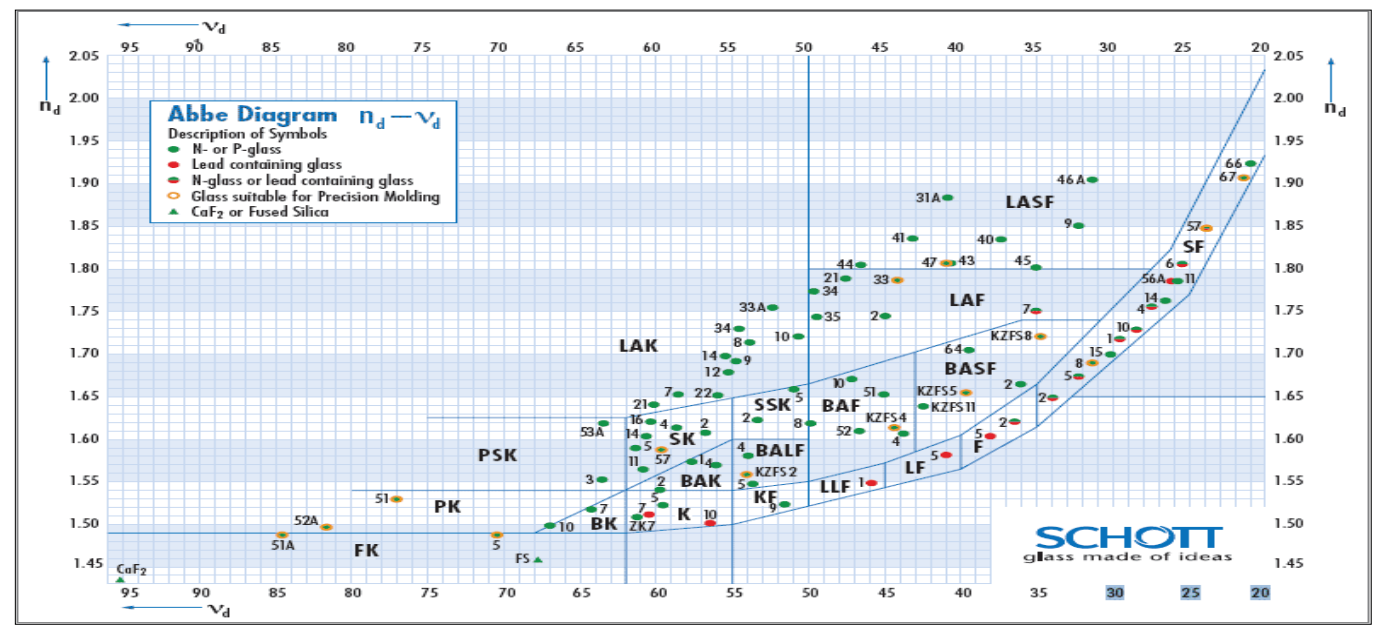

Figure 3.5: Abbe number glass map in variance with refractive index for different samples [69]

The available glass in the market and their respective refractive indices are clearly shown in Figure 3.5. From the Figure 3.5, we can select the material of desired properties for a specific purpose. Higher Abbe number means the glass with low refractive indices and vice versa for the glass of high refractive indices. 


\subsubsection{Consolidated Report:}

Figure 3.6 shows the above-discussed results of the birefringent cube in imaging in the consolidated format for explicit interpretation.

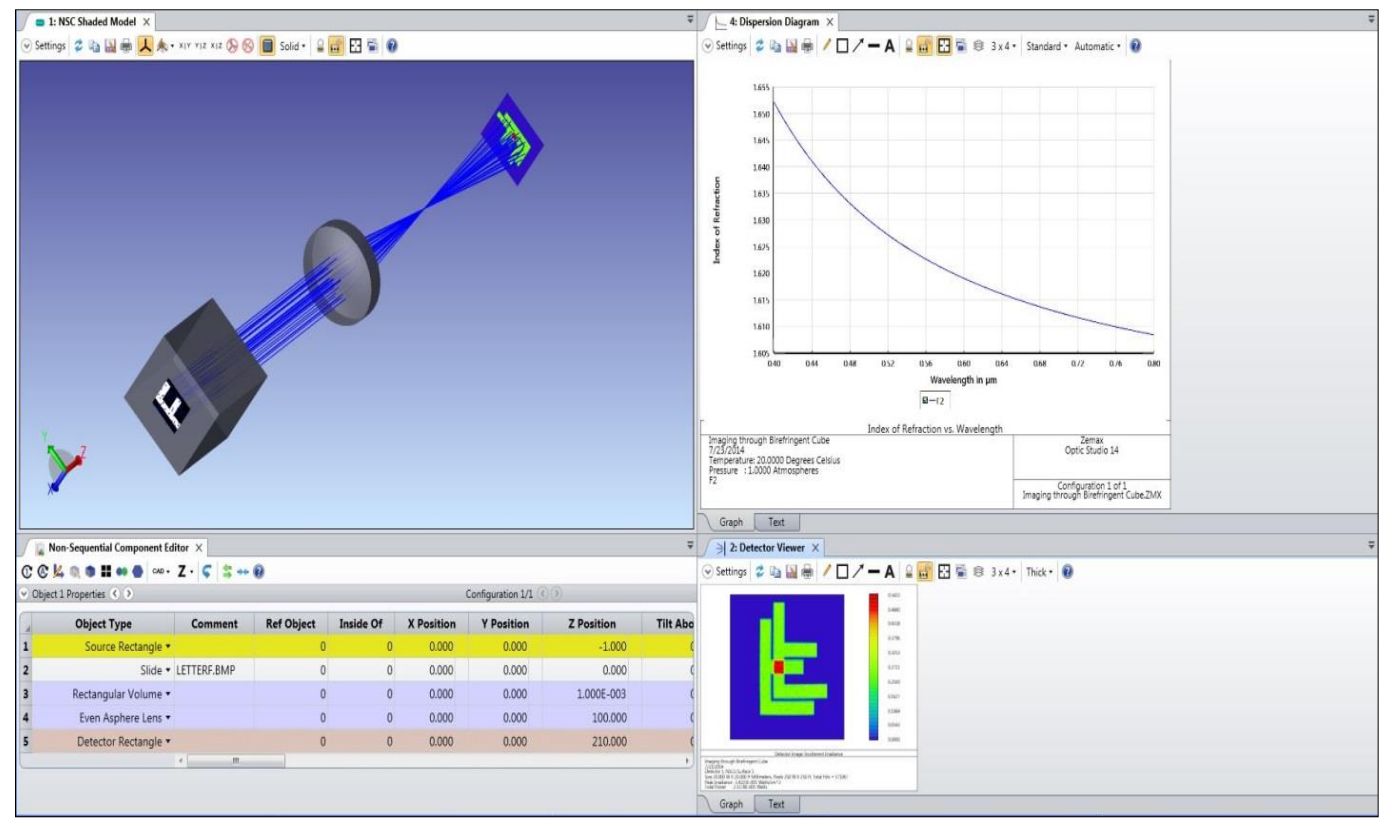

Figure 3.6: Consolidated simulation report of sample imaging through a birefringent cube

Hence, we can familiarise with various parameter involved in designing a birefringent cube and its transmittance properties on two different planes in ZEMAX. Although we were able to simulate intrinsic birefringence of the specimen, we are not able to simulate stress-induced birefringence values of an optical element which is a major limitation of this imaging software [70]. Thus, modelling of stress-induced birefringence is performed via finite element modelling software ABAQUS and compared with its theoretical and experimental results. 


\subsection{Stress-induced Birefringence modelling via Finite element analysis}

(ABAQUS)

There are various techniques for obtaining stress-induced birefringence which has been previously implemented in diode-pumped solid-state lasers. Crystals of $\mathrm{Nd}$ : $\mathrm{YVO}_{4}[71]$ have been deployed for inducing the desired birefringence of magnitude and direction. Similarly, stress-induced birefringent crystal Nd: YAG[72] in laser produces a controlled and variable cavity birefringence[73] which causes a variable frequency difference[74] between the two orthogonally polarised modes. Integrating stress-induced birefringence crystal in laser system has proved that they can be applied to measure retardation, force, displacement, velocity rather than being mere light sources. These systems utilise a sensing mechanism which is a point to point measurement method that consumes more time and has a low resolution. Here, we exploit an imaging system to analyse induced birefringence in an acrylic (Polymethyl methacrylate) specimen by applying stress in ABAQUS, modelling and simulation computer-aided design (CAD) software.

Polymethyl methacrylate specimen is modelled on computer-aided design (CAD) software by finite element method [70, 75, 76]. Material properties of PMMA like elastic, plastic characteristics and material density are meticulously registered in CAD. The gradual increment of force is applied to the specimen and its data values are recorded. The mechanical properties of the acrylic specimen as shown in Figure 3.7 is given below,

Density $(\rho)=1180 \mathrm{~kg} / \mathrm{m}^{3}$ 
Bending strength (fracture strength) $=134 \mathrm{MPa}$

Compressive strength $=165 \mathrm{MPa}$

Tensile strength (ultimate strength) $=62 \mathrm{MPa}$

Yield strength $($ yield point $)=60 \mathrm{MPa}$

Shear Modulus $(\mathrm{G})=1700 \mathrm{MPa}$

Young's Modulus $(\mathrm{E})=2450 \mathrm{MPa}$

Poisson's ratio $(v)=0.35$,
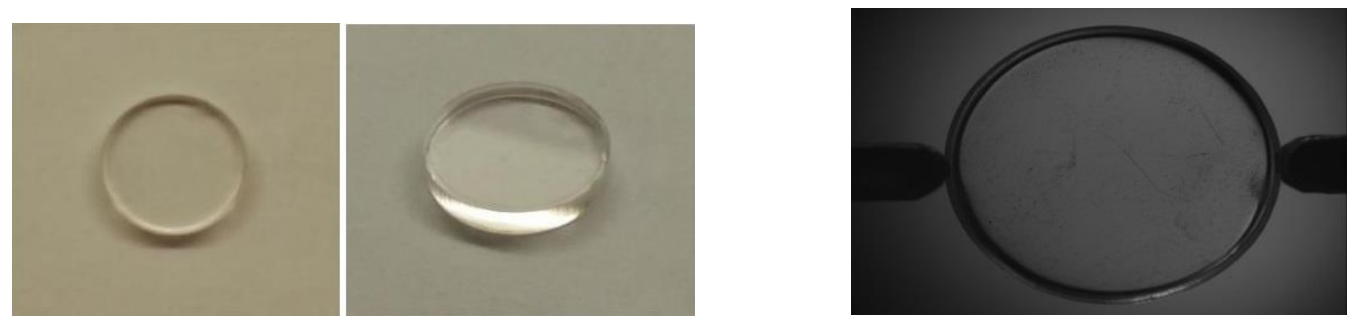

Figure 3.7: Poly-methyl methacrylate disk (Left), two-point compression loading (Right)

The boundary conditions were applied for a two-point compression loading based on finite element method. Uniform loading was applied to the specimen with a uniform increment to observe the material response at 0, 49, 98, 196, 294 and 392 $\mathrm{N}$. The stress intensity distribution is closely observed along the diameter of the specimen from both experimental and simulation results. The results from both approaches were found to be similar with minor discrepancies. Finally, theoretically calculated results were compared with both obtained experimental and simulation results and were found to be reasonably accurate and precise. Stress values obtained were transformed to birefringence values by a photoelastic 
calibration procedure. On photoelastic calibration, material fringe constant is calculated by observing retardation upon application of the known amount of stress.

\subsection{Simulation results}

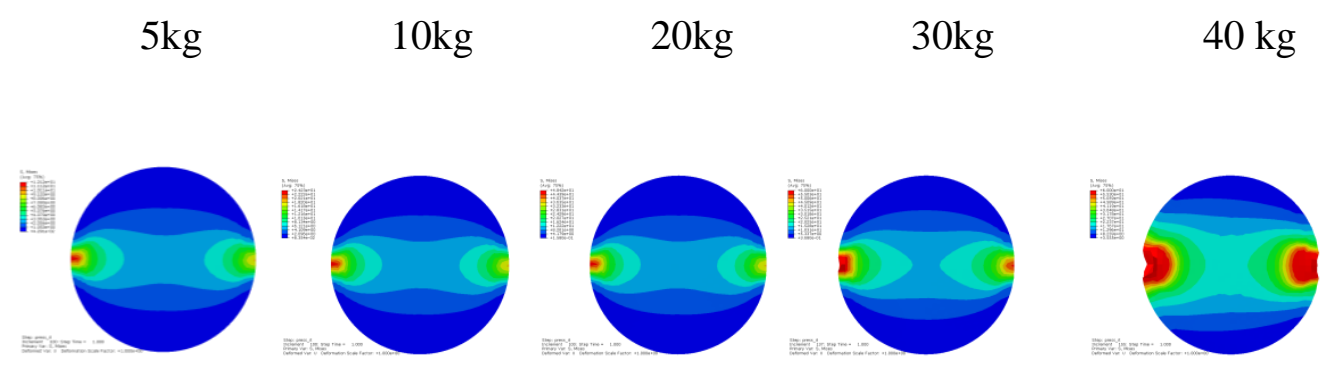

Figure 3.8: Stress values induced on specimen at different loads (simulation results)

The simulated stress values on specimen at different loads by finite element modelling in ABAQUS is shown in Figure 3.8. The increasing stress values are represented in the left side of each image based on colour progression. Simulated stress distribution on the pmma sample is similar to that of experimental stress distrubution as shown in Figure 3.9. There are minor discrepencies in the stress plot distribution because it cannot render residual stress (intrinsic birefringence) generated in the specimen due to thermal gradient, pressure gradient and others.

\subsection{Experimental results}

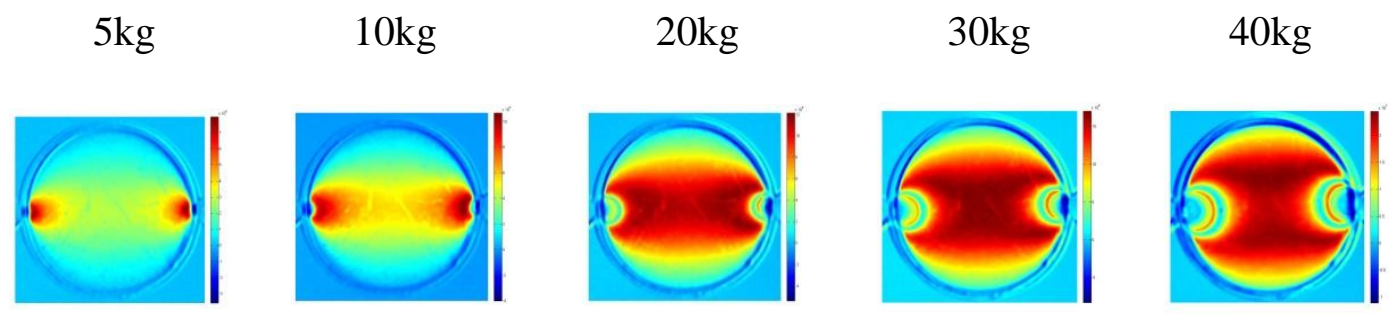

Figure 3.9: Stress values induced at different loads (experimental results) 
Stress magnitudes at different points were computed theoretically and experimentally as shown in Figure 3.8 and Figure 3.9. The values were observed to be in good agreement. Stress values are plotted along the vertical diameter of the sample with incremental stress. Theoretical, experimental and simulation results were plotted to observe the consistency and uniformity of the resultant values in the specimen. Ideally, materials are isotropic, i.e. refractive index is same and uniform throughout the material as considered in the theoretical calculation. In fact, there are minor non-uniformities in the material imparted by stress and temperature gradient during manufacturing.

$$
5 \mathrm{~kg} \quad 10 \mathrm{~kg} \quad 20 \mathrm{~kg} \quad 30 \mathrm{~kg} \quad 40 \mathrm{~kg}
$$
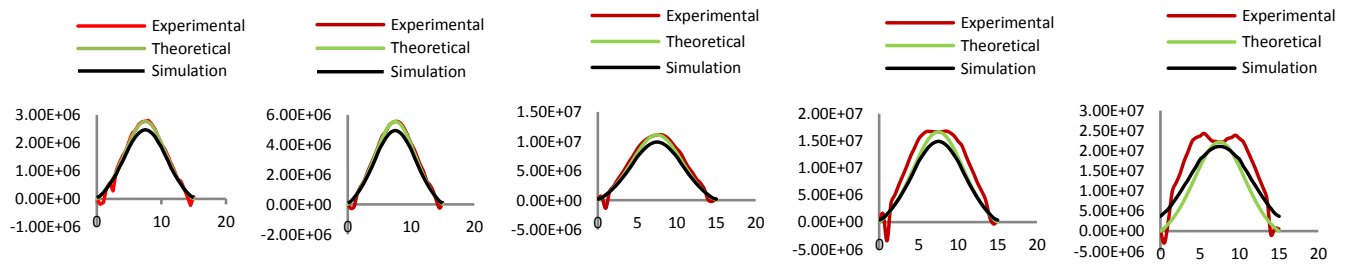

Figure 3.10: Stress values at different points along vertical diameter (y-axis) of specimen by different methods

The stress plot is dumbbell-shaped (normally distributed) and is the general nature of the curve for an isotropic material under compression loading at two opposite points. Stress values are maximum at the centre and gradually reduced along the edges with minor non-uniformities. The minor non-uniformities at the edges are due to maximum residual stress and temperature gradients due to abrupt temperature and pressure alteration in mould inserts openings. Stress values from solid mechanics, finite element modelling and photoelasticity $[77,78]$ are plotted and has a fine correlation as shown in the Figure 3.10. 


\subsection{Calibration (C)}

The wavelength of the light deployed in the experiment is $550 \mathrm{~nm}$. The calibration procedure is vital because it provides the basis for further analysis. If the specimen is not calibrated properly, it will lead to erroneous result and misinterpretation. The stress-optical coefficient (C) is associated with a photoelastic property of the specimen that can be determined by computing the fringe constant $\left(\mathrm{f}_{\sigma}\right)$ of the specimen. By applying a predetermined load $(\mathrm{P})$ and stress $\left(\sigma_{1}-\sigma_{2}\right)$, fringe constant of the specimen can be obtained. The fringe constant of the specimen is inversely proportional to the fringe order $(\mathrm{N})$ where thickness $(t)$ of the specimen remains constant.

$$
\sigma_{1}-\sigma_{2}=\frac{N f_{\sigma}}{t} \quad \rightarrow \quad f_{\sigma}=\frac{t\left(\sigma_{1}-\sigma_{2}\right)}{N}
$$

With incremental loading in the specimen, stress values gradually increase whereas photoelastic constant is invariant since it is the intrinsic specimen property.

$$
\begin{gathered}
\sigma_{1}-\sigma_{2}=\frac{8 P}{\pi t D}=\frac{\Delta \lambda}{2 \pi t C} \\
C=\frac{\lambda}{f_{\sigma}}
\end{gathered}
$$

A common calibration technique for finding the photoelastic constant requires a circular disk of diameter $\mathrm{D}$ and uniform thickness $\mathrm{t}$ subjected to a compression loading force $\mathrm{P}$ via loading equipment as shown in Figure 3.11. 


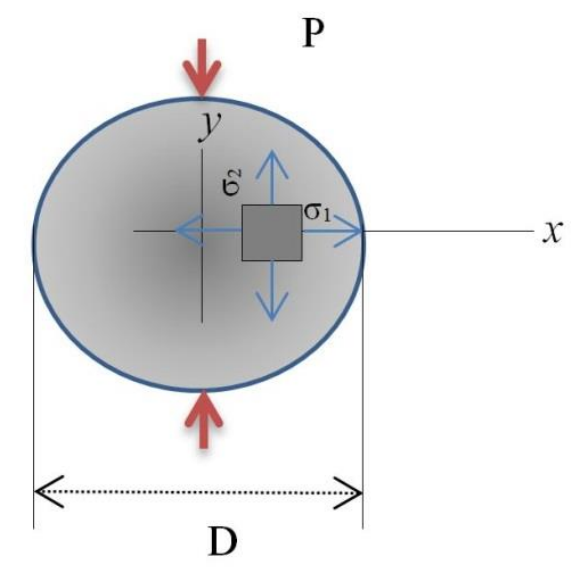

Figure 3.11: Compression loading in circular disk specimen

Horizontal and vertical stresses are inherent in the $\mathrm{x}$ and $\mathrm{y}$-axis of the specimen known as principal stresses. The circular disk is chosen because of its symmetric properties and henceforth no shear stress $\tau_{\mathrm{xy}}$ is present. From the Figure $3.11\left(\sigma_{1}\right)$ has positive values due to tensile effect whereas $\left(\sigma_{2}\right)$ has negative values due to compression effect that results $\left(\sigma_{1}-\sigma_{2} \geq 0\right)$ positive net stress values along the diameter of the sample. Thus from theory of photoelasticity $[9,77]$, the stresses along the $\mathrm{x}$-axis $\left(\sigma_{1}\right)$ and $\mathrm{y}$-axis $\left(\sigma_{2}\right)$ can be determined by the following equations,

$$
\begin{aligned}
& \sigma_{1}=\frac{2 \mathrm{P}}{\pi \mathrm{tD}}\left(\frac{1-\zeta^{2}}{1+\zeta^{2}}\right)^{2} \\
& \sigma_{2}=-\frac{6 \mathrm{P}}{\pi \mathrm{hD}} \frac{\left(1-\zeta^{2}\right)\left(1+\frac{1}{3} \zeta^{2}\right)}{\left(1+\zeta^{2}\right)^{2}},
\end{aligned}
$$

Where $\zeta=x / R=2 x / \mathrm{D}$. Maximum difference $\left(\sigma_{1}-\sigma_{2}\right)$ occurs at the centre along the horizontal diameter at $\zeta=0$. At this point, theoretical value of stress at the midpoint of the specimen is given by, 


$$
\sigma_{1}-\sigma_{2}=\frac{8 \mathrm{P}}{\pi \mathrm{h} \mathrm{D}}
$$

Combining this result with fundamental photoelastic relation,

$$
\begin{aligned}
& \frac{\mathrm{Nf}_{\sigma}}{\mathrm{h}}=\sigma_{1}-\sigma_{2}=\frac{8 \mathrm{P}}{\pi \mathrm{h} \mathrm{D}} \\
& \mathrm{f}_{\sigma}=\frac{8 \mathrm{P}}{\pi \mathrm{DN}}
\end{aligned}
$$

$$
\text { Where } \mathrm{N}=\frac{\Delta}{2 \pi} \text {, so } \mathrm{f}_{\sigma}=\frac{16 \mathrm{P}}{\Delta \mathrm{D}}
$$

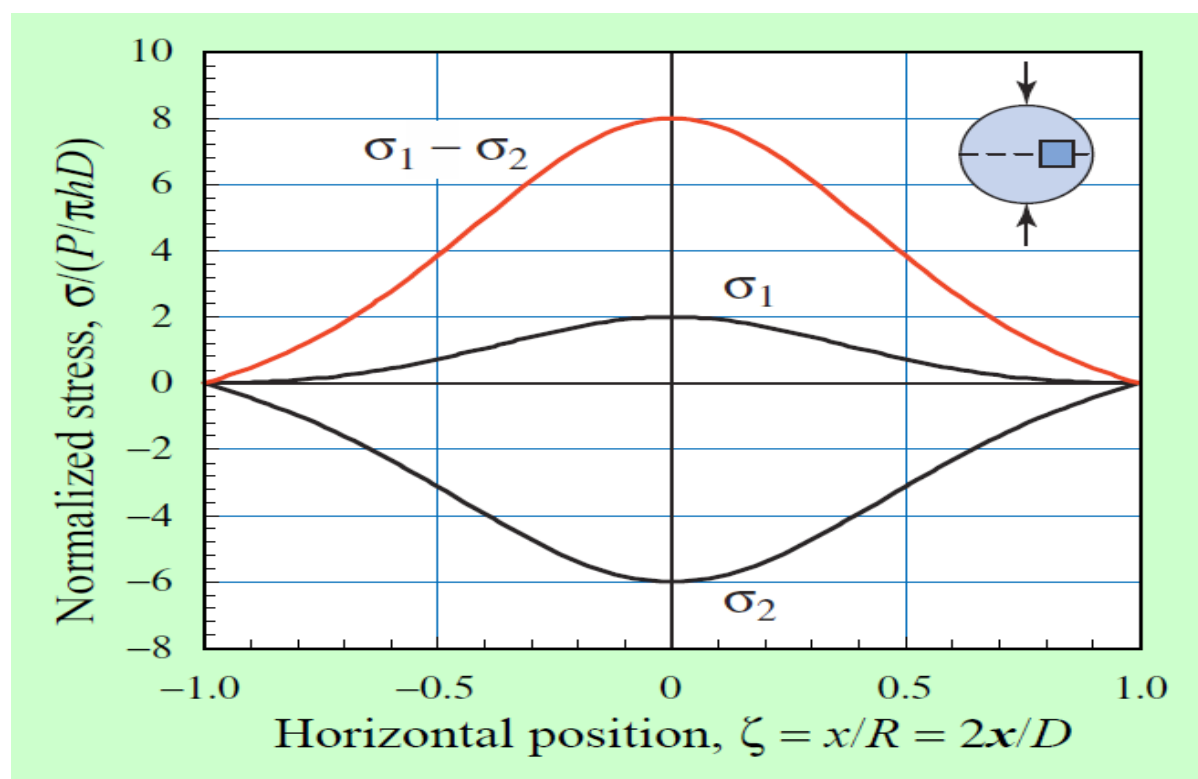

Figure 3.12: Stress distribution along vertical diameter of the specimen

The normalised stress distribution trend of the sample along vertical diameter based on the experiment is compliant with the stress distribution trend based on theoretical mechanics. From the Figure 3.12, we can observe that there is a 
negative compression effect along the vertical diameter whereas there is a tensile effect along the horizontal diameter of the circular specimen. We compute the photoelastic constant of the specimen based on their deflection in response to compressive or tensile stress. Thus, we can find out the fringe constant $(\mathrm{C})$ of the material for mapping stress values to birefringence values both in theoretical and simulation results.

$$
C=\frac{\Delta D \lambda}{16 P}
$$

\subsection{Birefringence values}
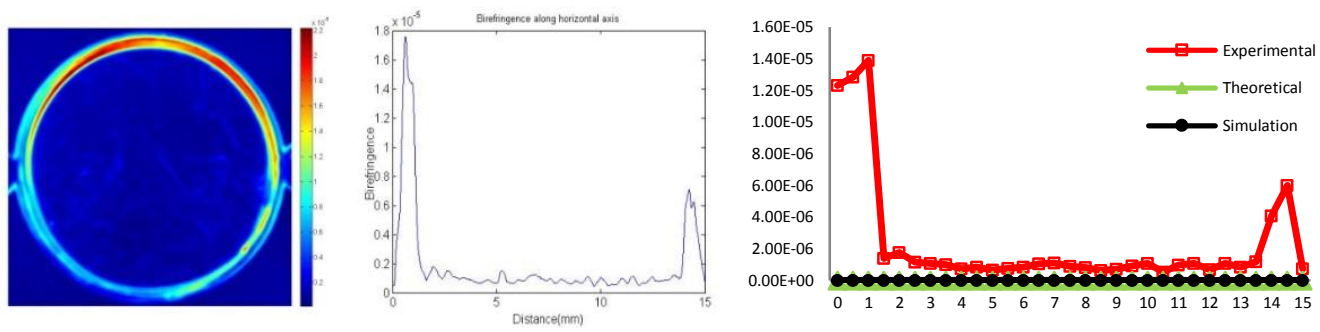

Figure 3.13: Birefringence values along vertical diameter (y-axis): Left (Intrinsic birefringence), Centre (Intrinsic birefringence plot) and Right (Comparison of birefringence plot)

Intrinsic birefringence values of the specimen are calculated as shown in Figure 3.13 (left) and compensated before comparing their theoretical, experimental and simulation values. It exists in specimen due to residual stress [69, 79] induced during manufacturing. The residual stress values are calculated by photoelastic method by nullifying the stress values present at no load condition from applied load condition. Obtained residual stress values are further transformed to birefringence values by mapping an obtained photoelastic constant during calibration. In theoretical and simulation results, the material is considered as isotropic i.e. stress-free where residual stress is entirely neglected. The abrupt 
birefringence values were observed at the material edges due to an imparted stress in the mould during ejection. It is almost impossible to get an isotropic material i.e. birefringence free material but annealing (slow temperature heating) and heat curing method release residual stress present in the specimen. Thus, this work introduces the intrinsic birefringence values of the specimen during manufacturing which has been neglected in simulation by finite element method. Despite minor deviations due to intrinsic birefringence, the stress curves on two-point compression loading are same. The low values birefringence are experimentally obtained by grey field photoelastic method [80]. Photoelastic constant are obtained at various points in the specimen to check the material non-uniformities. For calculating the photoelastic constant of the material, first fringe constant $\left(f_{\sigma}\right)$ value of material is calculated[81]. The fringe constant value is inversely proportional to the photoelastic constant value as in equation (3.11).

$$
\mathrm{f}_{\sigma}=\frac{\lambda}{\mathrm{C}}
$$

The LED light source implemented in the setup is of green colour $(550 \mathrm{~nm})$ with uniform intensity. The photoelastic coefficient $(\mathrm{C})$ of the specimen is calculated from the obtained birefringence values and the wavelength of the material. The calculated photoelastic coefficient of the material during the experiment is found to be $2.74 \times 10^{-12} \mathrm{~m}^{2} / \mathrm{N}$. These obtained values are mapped to residual stress to obtain birefringence values. Birefringence is defined by the following equation,

$$
\mathrm{Br}=\mathrm{n}_{1}-\mathrm{n}_{2}=\mathrm{C}\left(\sigma_{1}-\sigma_{2}\right)
$$


The stress induced birefringence at different loads are calculated and is shown in the Figure 3.14.

$5 \mathrm{~kg}$

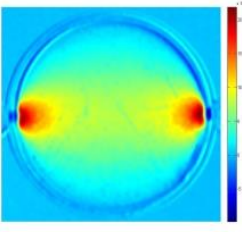

$10 \mathrm{~kg}$

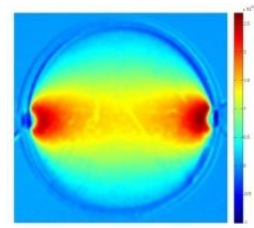

$20 \mathrm{~kg}$

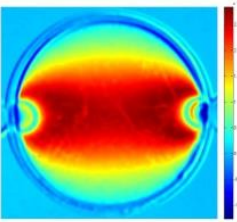

$30 \mathrm{~kg}$

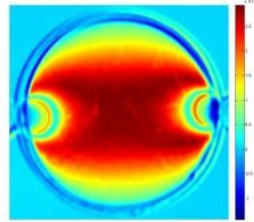

$40 \mathrm{~kg}$

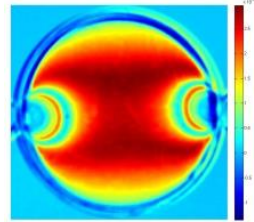

Figure 3.14: Birefringence values of the acrylic sample at different loads

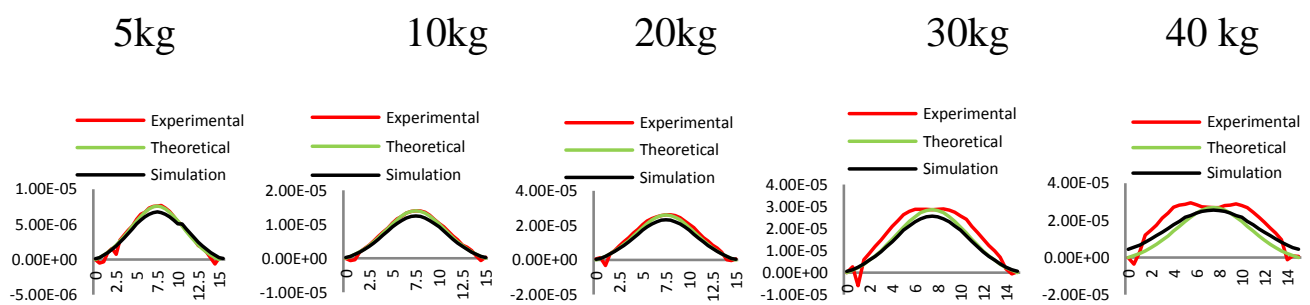

Figure 3.15: Birefringence plot along vertical diameter of the sample at increasing loads

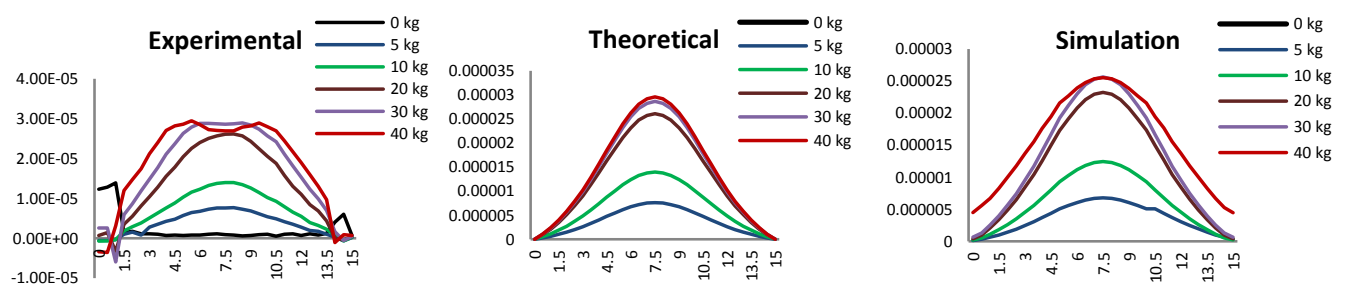

Figure 3.16: Comparison of birefringence values by various methods at increasing loads

The birefringence plot of the sample along vertical diameter at increasing load is compared to theoretical, experimental and simulation results as shown in Figure 3.15. Further, the results from theoretical, experimental and simulation results are compared with the gradual increase in stress in the sample along the vertical diameter as shown in Figure 3.16. Intrinsic birefringence is observed during 
manufacturing due to pressure flow discontinuity, thermal gradient, stress gradient [82] and many other reasons. In this work, we thoroughly investigated the intrinsic birefringence in the plastic specimen based on photoelasticity. The stress induced birefringence is compensated from intrinsic birefringence to obtain the actual birefringence. Intrinsic birefringence of the specimen is neglected theoretically; hence there are discrepancies in experimental, theoretical and simulation results. Thus, birefringence calculated at certain load will be altered if we don't incorporate intrinsic birefringence induced during manufacturing. The photoelastic coefficient of the specimen is calculated at various points and is found to be the same. The specimen at zero loading has intrinsic birefringence at the edges but has a maximum value at the centre point of incremental loading. Once loading exceeds elastic limit, material specimen manifests plastic characteristics. Birefringence values simultaneously increase with increase in load based in the theory of elasticity and is of linear nature. Thus, theoretical results based on solid mechanics, simulation based on finite element modelling (FEM) and experimental results are in good agreement. 


\subsection{Summary}

In this chapter, we have modelled the optical specimen in ZEMAX, optical simulation software. At ambient temperature and pressure, the light is traversed and the birefringence characteristics are observed in the specimen. The simulation software ZEMAX is suitable for lenses in an ideal condition but lacks stress induced birefringence module. Hence, we utilised finite element analysis (FEA) software for stress induced birefringence. PMMA sample is modelled via finite element analysis with gradually increasing loads to observe the stress induced birefringence and compared with the theoretical and experimental method. Since there is no imaging module on FEA software, stress values are mapped to birefringence values. The stress induced birefringence values at different loads is compared with theoretical and experimental values, both birefringence distribution trend and values are in good agreement. Stress induced birefringence measurement is appropriate tool for qualitative and quantitative inspection at industry level validated both by solid mechanics and finite element modelling.

Thus, there is essence of integrated system that can incorporate finite element modelling along with optical simulation. It should be able to simulate stress induced birefringence in the optical sample and its effect in imaging. The deterioration in imaging will be pre-analysed before real time application in laboratories, industries and research institute. Various states of optical sample under repeated application is highly monitored and pre-maintenance work can be scheduled to resolve forthcoming problems. In-situ birefringence measurement system is dire requirement in the manufacturing companies and industries where optical samples are intensively used in biological labs, optical research institutes and research and development $(\mathrm{R} \& \mathrm{D})$ for optoelectronics companies. 


\section{Chapter 4: Birefringence measurement of injection moulded optics}

Injection moulded optics are manufactured from injection moulding technology where plastic pellets are placed in the hopper of the system. The plastic pellets are crushed into a fine powder through an injection screw. The fine powder undergoes into a melting vat of polymer and solidifies in the mould insert by cooling procedure. During manufacturing of injection moulded components, it will undergo various procedure particularly temperature, pressure, an inertial and viscous process which renders non-uniformity in the sample micro-structure known as birefringence. Birefringence is prevalent in most of the injection moulded components thus need to have a meticulous inspection. Some injection moulded transparent plastic optics[67, 68] are used for imaging purpose, birefringence in plastic optics sample generates inexact result. Birefringence measurement system identifies defects, cracks and non-uniformities in the sample vital for qualitative and quantitative inspection in plastic optics.

\subsection{Introduction}

Injection moulding is a standard technology for mass manufacturing of plastic optics. The benefits of injection moulding mechanism are inexpensive manufacturing cost [8] and mass production. Optical moulded materials are deemed to be precise in size, shape and form. Unrestricted mould shrinkage, stress generated distortion[69], temperature gradient and material characteristics are the problems that causes optical aberrations in moulded plastic optics[83]. Residual stress[84], which also affects the functional characteristic of plastic samples is 
being investigated[80, 85]. This study aims to do a detailed study in the birefringence and residual stress that causes a difference in different injection moulded components[43, 79]. Low Birefringence Polariscope (LBP) developed by a local company[81] is deployed for quantifying the birefringence and residual stress distribution. The measured values are compared to the commercial system for validation and verification purpose. Phase shift polariscope is preferable among others attributed to its high resolution and whole field measurement[86] capability. It can be designed in an ellipsometric[87] [88] configuration to serve the purpose.

\subsubsection{Micro-plate material}

Qualitative as well as quantitative inspection of various microplates will be conducted[85] via birefringence measurement system. Polystyrene, polypropylene and cyclo-olefin polymers are frequently used materials for manufacturing microplates. Polystyrene microplates are utilised for cell culture and calorimetric assays[89]. Usually, in compound storage or assays where chemical interaction is the altering factor, polypropylene is preferred. Economic and non-sterile assay plates are made up of flexible vinyl which is commonly used microplates. However, regarding usage and application, polystyrene and polypropylene are mostly used as micro plates[90]. Having transparent and superior optical properties, polystyrene is predominantly used where precision is a critical issue. Polypropylene is known for its chemical and thermal stability, least autofluorescence and excellent transparency designed for UV range. 


\subsection{Birefringence measurement by phase shifting method}

The schematic of the LBP is shown in Figure 4.1 based on a phase shift algorithm[91]. In Phase shifting photoelasticity, phase-shifted intensity images are recorded and processed to determine principal stress along with its orientation.

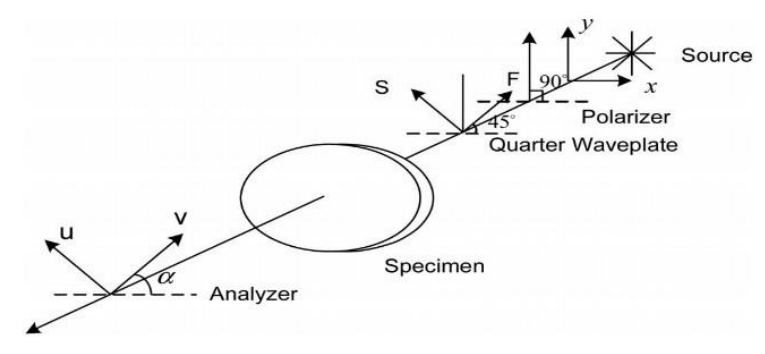

Figure 4.1: Schematic diagram of phase shift polariscope

Considering the average intensity of light as $\mathrm{I}_{\mathrm{a}}$, which is constant during the entire experiment, a four-step phase shifting can be applied to calculate the unknown values $\Delta$ (phase difference) and $\beta$ (phase direction). Table 4.1 outlines the optical arrangement of the system and its related equations.

\begin{tabular}{|r|c|c|}
\hline Steps & Analyzer orientation $(\alpha)$ & Intensity equation \\
1 & 0 & $\mathrm{I}_{1}=\mathrm{I}_{\mathrm{a}}(1-\sin \Delta \sin 2 \beta)$ \\
2 & $\pi / 4$ & $\mathrm{I}_{2}=\mathrm{I}_{\mathrm{a}}(1+\sin \Delta \cos 2 \beta)$ \\
3 & $\pi / 2$ & $\mathrm{I}_{3}=\mathrm{I}_{\mathrm{a}}(1+\sin \Delta \sin 2 \beta)$ \\
4 & $3 \pi / 4$ & $\mathrm{I}_{4}=\mathrm{I}_{\mathrm{a}}(1-\sin \Delta \cos 2 \beta)$ \\
\hline
\end{tabular}

Table 4.1: Intensity equations for phase shift polariscope

Retardation values and its direction are as follows,

$$
\begin{aligned}
& \Delta=\frac{1}{2 I_{a}}\left(\left(I_{3}-I_{1}\right)^{2}+\left(I_{4}-I_{2}\right)^{2}\right)^{1 / 2} \\
& \beta=\frac{1}{2} \tan ^{-1}\left(\frac{I_{1}-I_{3}}{I_{4}-I_{2}}\right)
\end{aligned}
$$


Birefringence induced due to change in phase is given by,

$$
n_{1}-n_{2}=\frac{\Delta \times \lambda}{2 \Pi t}
$$

Thus, equation 4.3 measures birefringence induced in the injection moulded samples of varied sizes and shapes as shown in Figure 4.2.
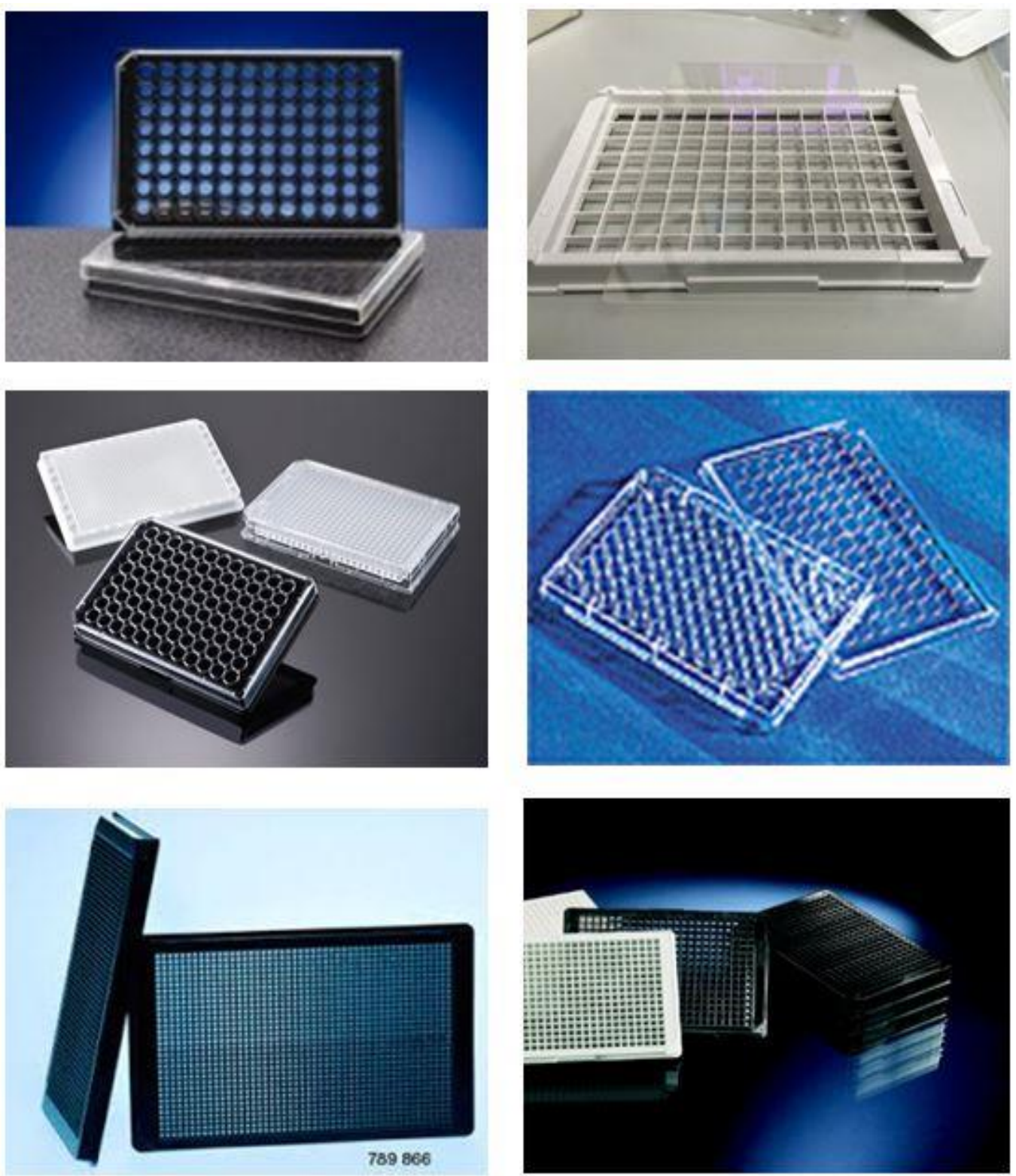

Figure 4.2: Injection moulded specimen (microplates) used in experiment 


\section{Part Geometry}

The parts are first classified according to their shape and size for the design of the experiment. Further, they are classified based on their manufacturing process. It is crucial to classify parts based on manufacturing process and size for the easy sampling selection and product verification. Parts involved in the measurement are of circular and rectangular shape depending on their type as mentioned in

Table 4.2:

\begin{tabular}{|c|c|c|c|c|}
\hline Type & Material & Shape & Diame ter (mm) & Thickness (mm) \\
\hline \multirow{7}{*}{ 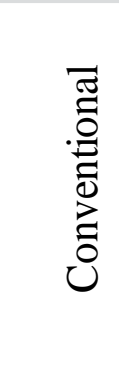 } & BD falcon & Circular & 7 & 0.19 \\
\hline & COP & Rectangular & $8 \times 8$ & 0.76 \\
\hline & Corning & Rectangular & $8 \times 8$ & 1 \\
\hline & Grenier & Rectangular & $3.50 \times 3.50$ & 0.19 \\
\hline & Nunc 96 & Circular & 4.5 & 1.2 \\
\hline & Nunc 384 & Circular & 5 & 1.2 \\
\hline & Perkin Elmer & Circular & 6.5 & 0.76 \\
\hline \multirow{14}{*}{$\frac{\Xi}{\frac{\Xi}{d}}$} & $1 \mathrm{a}$ & Circular & 2.5 & 1 \\
\hline & $1 b$ & Circular & 2.5 & 1 \\
\hline & $1 \mathrm{c}$ & Circular & 2.5 & 1 \\
\hline & $1 d$ & Circular & 2.5 & 1 \\
\hline & $1 \mathrm{e}$ & Circular & 2.5 & 1 \\
\hline & \multicolumn{4}{|c|}{ Advanced Plasma me thod } \\
\hline & BiomatCOC & Circular & 3.5 & 1.25 \\
\hline & $\operatorname{COC} 24 w$ & Rectangular & $11 \times 11$ & 1.25 \\
\hline & CR8 & Rectangular & $11 \times 11$ & 1.25 \\
\hline & G2CR8 & Circular & 2 & 1.25 \\
\hline & COC1DOC1 & Circular & 2 & 1.25 \\
\hline & $\mathrm{COC} 2$ & Circular & 3.5 & 1.25 \\
\hline & PS1 & Circular & 2 & 1.25 \\
\hline & PS2 & Circular & 2 & 1.25 \\
\hline
\end{tabular}

Table 4.2: Dimensions of specimen plates 


\subsection{Design of Experiment}

There are twenty specimens; every microplate birefringence measurements were taken multiple times for reproducibility of the results. The resolution of the measurement system is five (5) microns $(\mu \mathrm{m})$. Twenty different microplates specimen were qualitatively and quantitatively assessed for sample selection required for further usage in biological sampling. Samples manufactured via conventional and modern manufacturing method has been measured and analysed by birefringence measurement system. There are two different microplates employed for the investigation i.e. conventional and modern. Various circular and rectangular shaped microplates are measured, where most of the specimens are circular. Among twenty specimens, fifteen of them are circular specimens whereas five of them are rectangular specimens. Specimens consist of varied materials like polystyrene, polypropylene and cyclo-olefin materials as shown in Table 4.3 along with their material property. The most chosen material is acrylic sample because of its affordable cost and high transmissivity compared to other acrylic materials. Other materials are relatively more expensive and easily affected by thermal and pressure gradient. Acrylic because of its moderate cost and high transmissivity is the most used plastic materials for domestic, biological and industrial purpose.

\begin{tabular}{|c|l|r|r|r|}
\hline S.N. & Materials & Refractive Index $(\boldsymbol{\mu})$ & Transmissivity $(\boldsymbol{\%})$ & Thermal Expansion $\left(\mathbf{1 0}^{-\mathbf{5}} \mathbf{0}^{\mathbf{0}} \mathbf{)}\right.$ \\
\hline 1 & Acrylic & 1.497 & $85-91$ & $6.0-7.0$ \\
\hline 2 & Cycloolefin polymer (COP) & 1.537 & $87-92$ & $6.0-7.0$ \\
\hline 3 & Cycloolefin Copolymer (COC) & 1.54 & 92 & $6.0-8.0$ \\
\hline 4 & Polycarbonate & 1.593 & 92 & $6.6-7.0$ \\
\hline 5 & Polystyrene & 1.604 & 92 & 6.74 \\
\hline 6 & Polysulfone & 1.634 & 84 & 5.4 \\
\hline
\end{tabular}

Table 4.3: Properties of biological microplates 


\subsection{Birefringence and Retardation analysis}

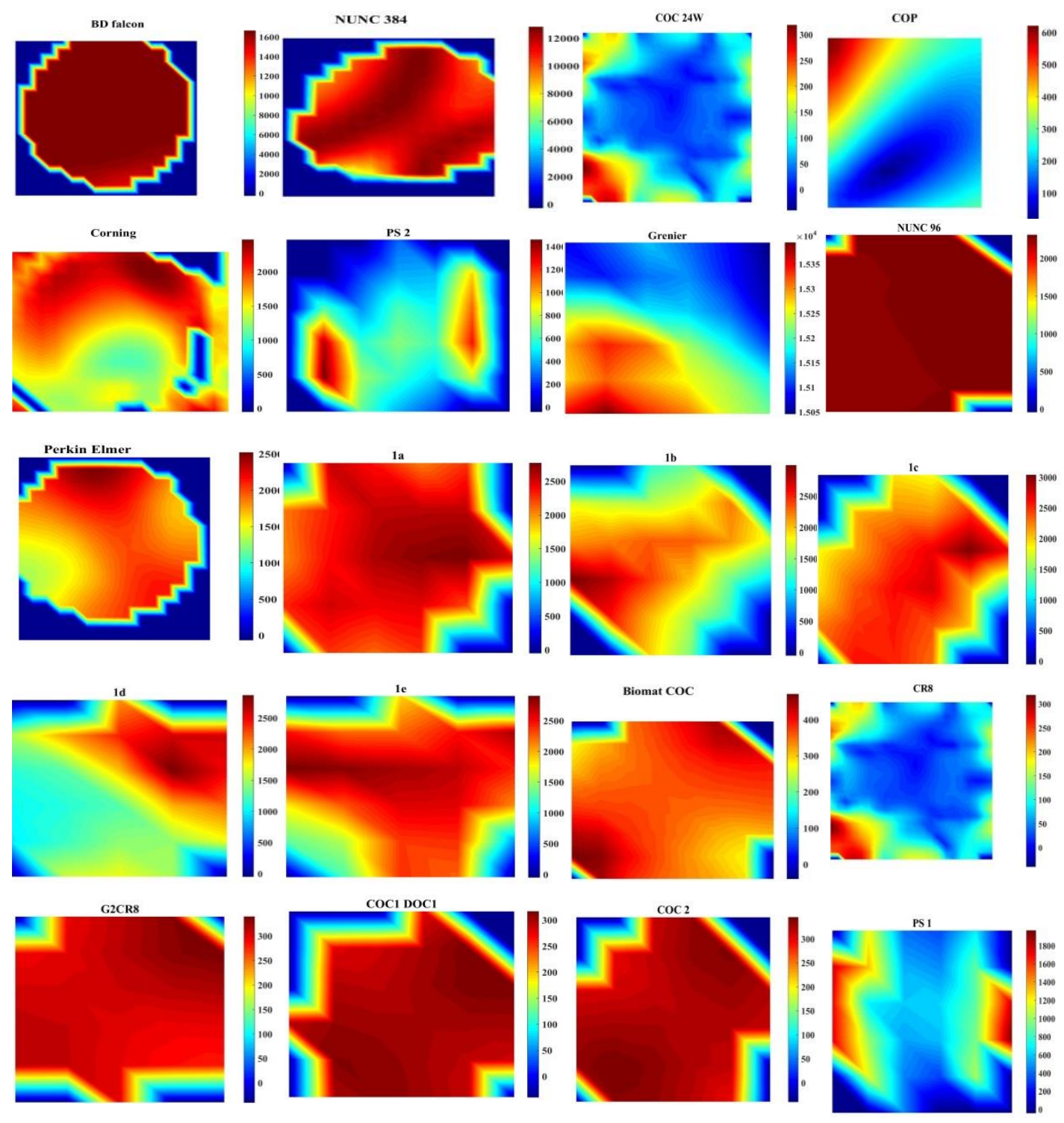

Figure 4.3: Birefringence values $(\mathrm{nm} / \mathrm{cm})$ of various micro plates specimen

Figure 4.3 represents the birefringence values of various microplate specimen conducted in the experiment. BD Falcon has higher birefringence whereas COC 24W has the least birefringence. Theoretically, optically transparent plates are distortion free and without temperature gradient which produces isotropic material of uniform refractive index. During manufacturing, it encounters various issues like temperature gradient, pressure gradient and other ambient factors which 
induce deformation in the material microstructure. It creates varying particle density along the axes of the microstructure. Varying particle density in axes of the material varies propagation of light velocity known as birefringence of the material. It deters the image quality and provides altered birefringence values during microplate inspection. It modifies the intrinsic polarisation state of a beam splitting into two orthogonal components of different propagation velocities, i.e. ordinary and extraordinary rays; explained by equation (4.3). This alteration is examined with an efficient technique known as photoelasticity. The photoelastic coefficient is considered to be constant for the isotropic specimen but there is an intrinsic retardation which engenders non-uniformity in the material. Retardation comprises of an amount of light retarded through the specimen in different magnitude due to birefringence which is proportional to stress and temperature variation by material microstructure[79]. Hence, uniform and low birefringence allow us to get fine discernment, rapid scintillation calculation and high-resolution microscopy during imaging[92]. High birefringence makes reading or data values inaccurate. For materials of higher birefringence, the colour range will be limited as illustrated in Figure 4.3. Thus, there is the essence of qualitative and quantitative inspection of the proper birefringence measurement system. Reliable and accurate inspection of microplates can be assessed by the low birefringence measurement system. Twenty readings were taken on each sample and average birefringence was calculated to ensure precision and accuracy in the measurement system. This inspection will lead to select the samples qualitatively for higher and lower birefringence values based on their results. 


\subsection{Detailed table on Birefringence and Retardation of micro- plates}

The comparison of birefringence and retardation values of the sample measured by the birefringence measurement system are given in Table 4.4. These values are crucial for qualitative and quantitative selection of the specimen.

\begin{tabular}{|c|c|c|}
\hline Specimen & Birefringence $(\mathrm{nm} / \mathrm{cm})$ & Retardation (nm) \\
\hline BD Falcon & 16470.7 & 312.94 \\
\hline COP & 257.61 & 19.57 \\
\hline Corning & 1727.21 & 172.72 \\
\hline Grenier & 15224.36 & 289.26 \\
\hline NUNC 96 & 2279.71 & 273.56 \\
\hline NUNC 384 & 11459.08 & 217.74 \\
\hline Perkin Elmer & 1902.15 & 144.56 \\
\hline $1 \mathrm{a}$ & 2389.16 & 238.92 \\
\hline $1 b$ & 1912.58 & 191.26 \\
\hline $1 \mathrm{c}$ & 2298.74 & 229.87 \\
\hline $1 d$ & 1750.4 & 175.04 \\
\hline $1 \mathrm{e}$ & 2222.11 & 222.21 \\
\hline Biomat COC & 365.85 & 45.73 \\
\hline COC 24w & 82.88 & 67.41 \\
\hline CR8 & 82.88 & 10.36 \\
\hline G2 CR8 & 307.14 & 38.39 \\
\hline COC1DOC1 & 299.7 & 37.46 \\
\hline $\mathrm{COC} 2$ & 324.32 & 40.54 \\
\hline PS1 & 926.24 & 115.78 \\
\hline PS2 & 668.2 & 83.52 \\
\hline
\end{tabular}

Table 4.4: Birefringence and retardation values of micro plate specimen 


\subsection{Calibration $(C)$}

The calibration procedure is performed to assess the photoelastic constant of the material. Two- point compression calibration method is widely adopted procedure in engineering mechanics to find the photoelastic constant of the specimen. Two-point vertical loading in the specimen is performed through compact loading machine as shown in Figure 4.4. The compact loading machine is designed in the lab to integrate into the polariscope. The force will be applied longitudinally on the sample to observe the photoelastic behaviour in the acrylic specimen. In the photoelastic specimen, we applied the diametric compression on specimen i.e. two-point loading of $3 \mathrm{~kg}, 4 \mathrm{~kg}$ and $5 \mathrm{~kg}$ subsequently.

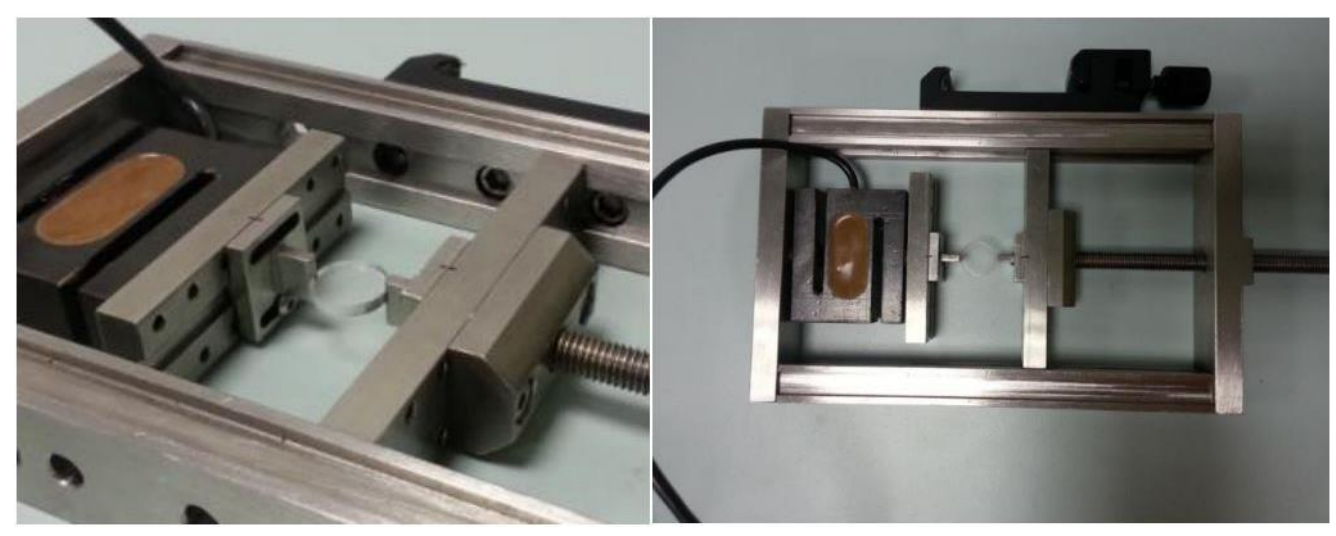

Figure 4.4: Setup for two-point loading of circular disk in compact loading machine

\subsubsection{Compact loading machine and load cell for calibration}

The load cell with a compact loading machine as shown in Figure 4.5 is deployed to induce the desired force on the sample for calibration purpose. The test specimen is inserted in the centre of the clamp and a compressive force is applied upon rotation of the handle. The load cell as the transducer is used to measure the deformation in the form of electrical signal due to changes in electrical resistance 
in the wire. The output electrical signal will be scaled to an amount of compressive force applied to the specimen.

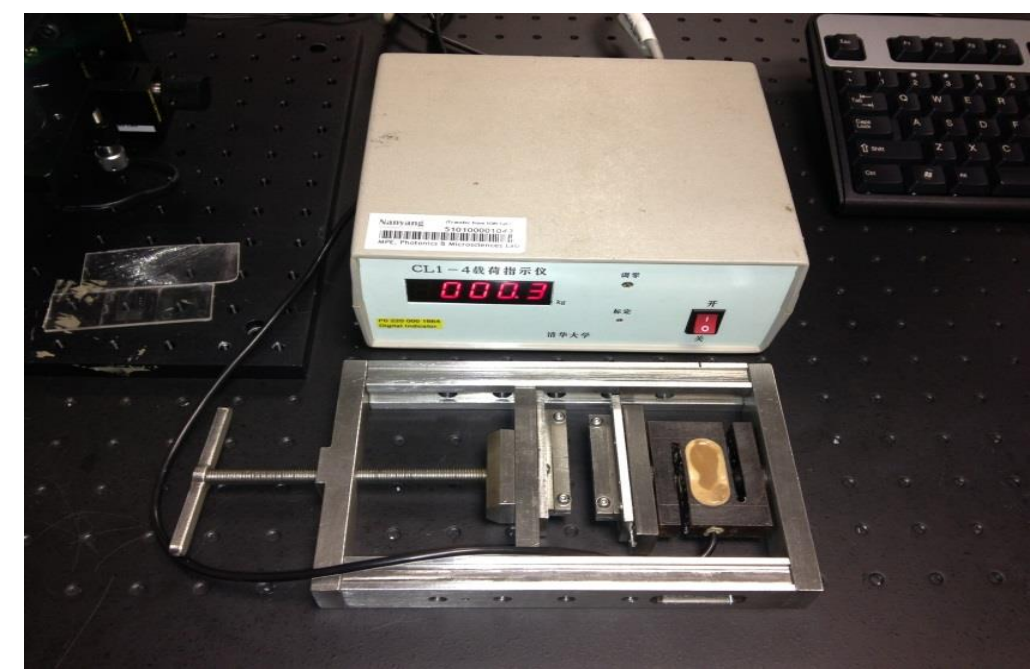

Figure 4.5: Compact loading machine apparatus with load cell

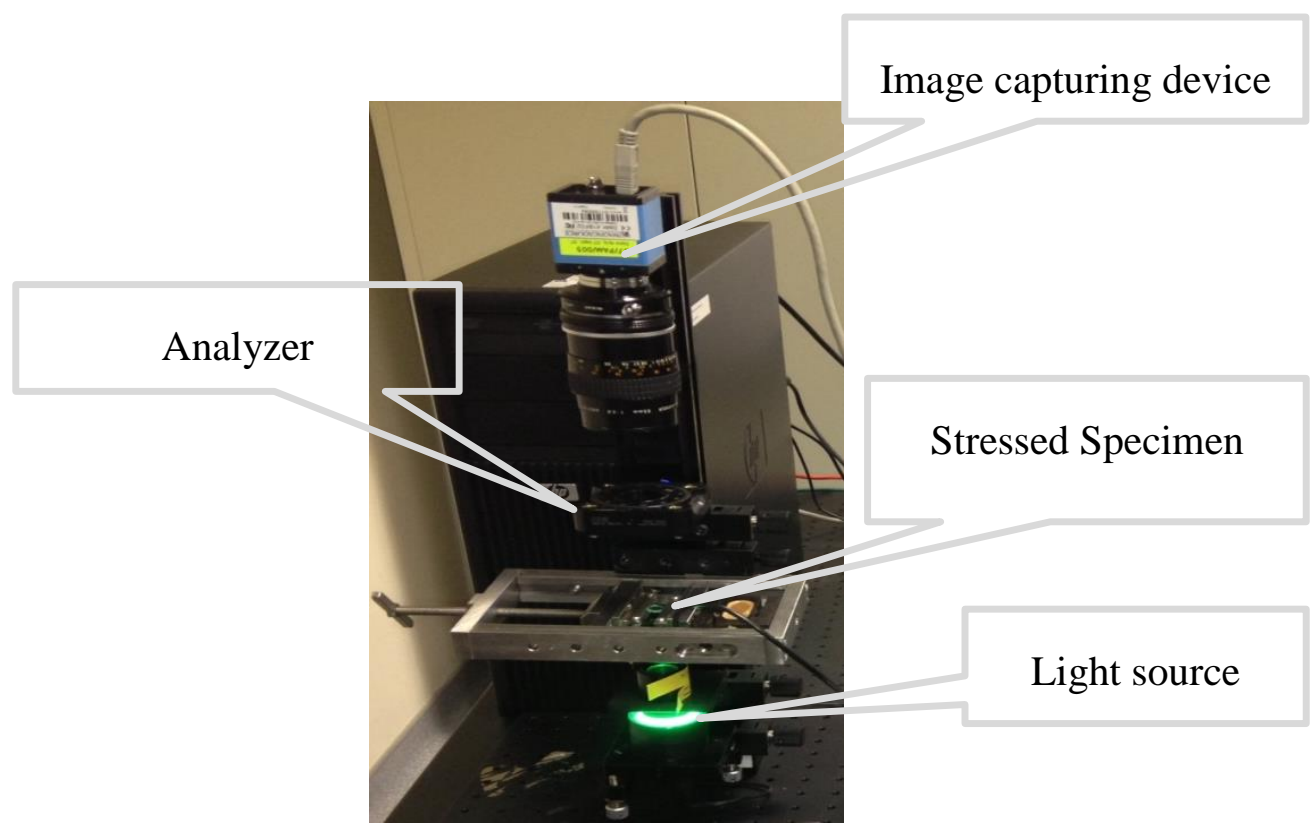

Figure 4.6: Optical elements of phase shift polariscope measurement system

The photoelastic constant of the material is calculated by applying a known amount of stress and observing retardation or phase difference $(\Delta)$ exhibited by a material. The light source employed in the system is of wavelength $550 \mathrm{~nm}$ and 
diameter of the specimen is $24.76 \mathrm{~mm}$. A typical calibration sample is a disk under compression as shown in Figure 4.6.

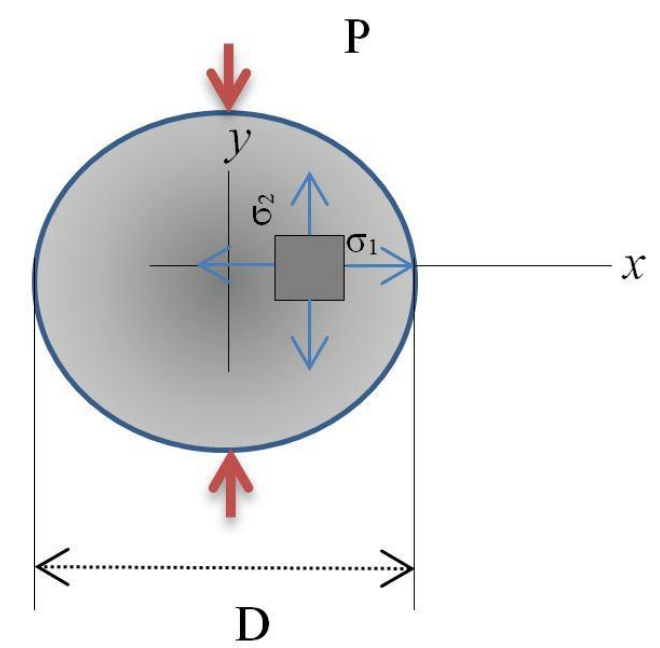

Figure 4.7: Compression loading in circular disk specimen for calibration purpose Horizontal and vertical stresses are present in the $\mathrm{x}$ and $\mathrm{y}$-axis particularly known as principal stresses. The circular disk is chosen because of its symmetric properties and absence of shear stress values $\left(\tau_{\mathrm{xy}}\right)$. From Figure 4.7, $\sigma_{1}$ has positive values due to a tensile effect whereas $\sigma_{2}$ has negative values because of a compression effect which results $\left(\sigma_{1}-\sigma_{2}\right) \geq 0$. Thus, from the theory of photoelasticity $[9,77]$, the stress along the $\mathrm{x}$-axis and $\mathrm{y}$-axis can be determined by the following equations,

$$
\begin{aligned}
& \sigma_{1}=\frac{2 \mathrm{P}}{\pi \mathrm{tD}}\left(\frac{1-\zeta^{2}}{1+\zeta^{2}}\right)^{2} \\
& \sigma_{2}=-\frac{6 \mathrm{P}}{\pi \mathrm{hD}} \frac{\left(1-\zeta^{2}\right)\left(1+\frac{1}{3} \zeta^{2}\right)}{\left(1+\zeta^{2}\right)^{2}},
\end{aligned}
$$


Where $\zeta=x / \mathrm{R}=2 x / \mathrm{D}$. Maximum difference $\left(\sigma_{1-} \sigma_{2}\right)$ occurs at the centre along the horizontal diameter at $\zeta=0$. At this point, theoretical value of stress at the midpoint of the specimen is given by,

$$
\sigma_{1}-\sigma_{2}=\frac{8 \mathrm{P}}{\pi \mathrm{h} \mathrm{D}}
$$

Combining this result with fundamental photoelastic principal gives,

$$
\begin{aligned}
& \frac{\mathrm{Nf}_{\sigma}}{\mathrm{h}}=\sigma_{1}-\sigma_{2}=\frac{8 \mathrm{P}}{\pi \mathrm{h} \mathrm{D}} \\
& \mathrm{f}_{\sigma}=\frac{8 \mathrm{P}}{\pi \mathrm{DN}}
\end{aligned}
$$

Where $\mathrm{N}=\frac{\Delta}{2 \pi}$, so

$$
\mathrm{f}_{\sigma}=\frac{16 \mathrm{P}}{\Delta \mathrm{D}}
$$

Thus, we can find out the fringe constant of the material for mapping stress values to birefringence values both in theoretical and simulation results. For specimens prepared using injection moulding, the calibration sample possesses residual birefringence also known as the intrinsic phase difference of the specimen. Hence to calibrate such specimens, we need to first determine the phase difference $\left(\Delta_{0}\right)$ at no load state as in Figure 4.9 at $0 \mathrm{~kg}$. The phase difference at gradually loaded $\left(\Delta_{\mathrm{L}}\right)$ state (i.e. $\left.3 \mathrm{~kg}, 4 \mathrm{~kg}, 5 \mathrm{~kg}\right)$ as shown in Figure 4.10 is determined to observe the phase difference upon application of force. The compensated phase difference is the real phase difference values of the specimen also known as intrinsic retardation in the specimen. After calibration, we can compute the fringe constant of the material. The fringe constant of the material is also known as the photoelastic constant of the specimen. This value is mapped to calculated phase difference values to acquire the birefringence values of microfluidic channels. 
Horizontal stresses along the $\mathrm{x}$-axis are present in the specimen which is known as principal stress. Shear stress $\tau_{\mathrm{xy}}$ is absent in the specimen since we chose symmetric specimen (circle). Thus from the theory of photoelasticity [9, 77], stress optic coefficient of the material is determined as,

$$
C=\frac{\Delta D \lambda}{16 P}
$$

On calibration, we obtain the fringe constant of the material. After that, the stress values are mapped to birefringence values. The mapping of stress values to birefringence values is governed by the following equation,

$$
n_{1}-n_{2}=C\left(\sigma_{1}-\sigma_{2}\right)
$$

The photoelastic constant $(\mathrm{C})$ of the sample is obtained from the calibration procedure. Obtained stress values are mapped to birefringence values, once photoelastic constant of the material is determined.

\subsubsection{PMMA (Poly-methyl Methacrylate)}

The load was applied using a load cell as shown in Figure 4.7 and the obtained images were processed to find the photoelastic constant of the sample. The intrinsic phase difference values were subtracted from the specimen to get actual phase difference values by applying a load in the incremental order. The protruded rheological sample specimen with a schematic diagram for two- point loading is represented in Figure 4.8. The intrinsic birefringence distribution along with induced birefringence is represented in Figure 4.9. We plot the effect of load on the centre line along the vertical diameter as in Figure 4.10 and Figure 4.11 with and without intrinsic birefringence (residual stress). The fringe constant of the material is calculated at various loadings to observe the consistency in the results. 


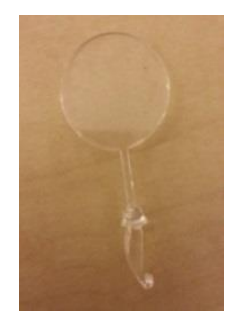

Load

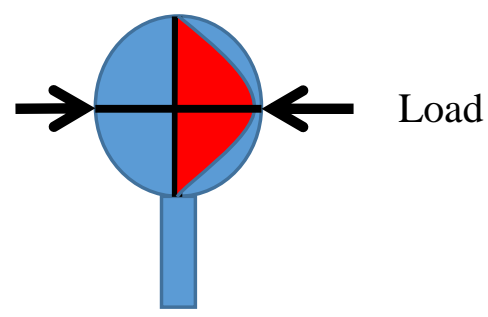

Figure 4.8: Circular protruded acrylic specimen (left), schematic diagram (right)

Here, fringe constant $f_{\sigma}=\frac{\lambda}{C}$, where the fringe constant is calculated from equation (4.9). Since we know the fringe constant by calibration method as described in the calibration procedure, we can assess the material (photoelastic) constant value (C). For specimens prepared using injection moulding, the calibration sample possesses residual birefringence also known as the intrinsic phase difference of the specimen. The compensated phase difference value is the real phase difference value of the specimen. After calibration, we determine the fringe constant of the material. Then, we map the phase difference values to birefringence values by the aid of photoelastic constant values of the specimen.

$0 \mathrm{~kg}$

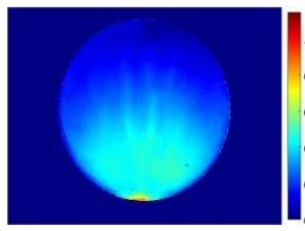

$3 \mathrm{~kg}$

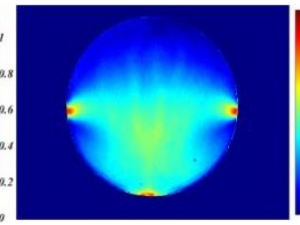

$4 \mathrm{~kg}$

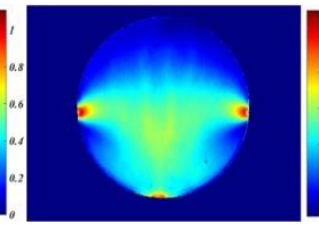

$5 \mathrm{~kg}$

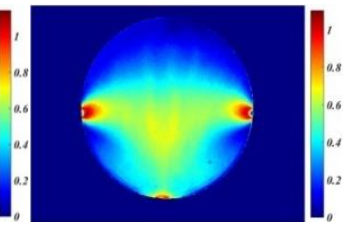

Figure 4.9: Induced phase difference values at different loads in an acrylic disk
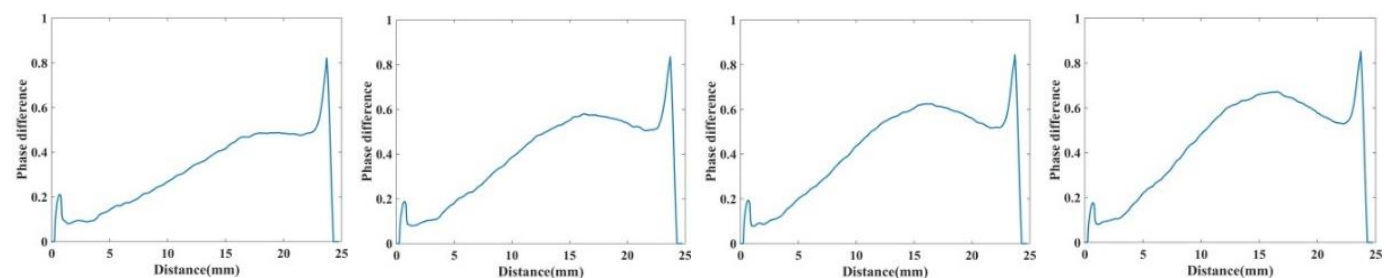

Figure 4.10: Induced phase difference of an acrylic disk at different loads along vertical diameter 


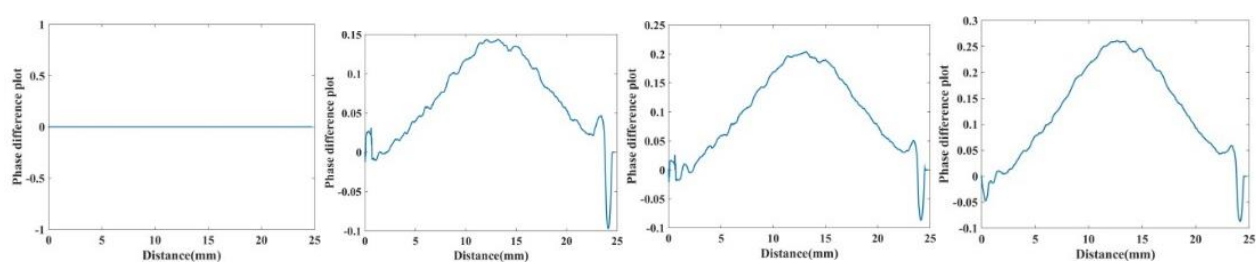

Figure 4.11: Induced phase difference of an acrylic disk on compensating intrinsic birefringence

Thus, phase difference value is crucial to obtain the photoelastic constant (C) of the specimen as in equation (4.10). Thus, the photoelastic constant obtained for the specimen at three different loading ( $3 \mathrm{~kg}, 4 \mathrm{~kg}, 5 \mathrm{~kg})$ is stated below.

Photo elastic Coefficient (C) $\quad 5.11 \times 10^{-12} \mathrm{~m}^{2} / \mathrm{N} \quad 5.17 \times 10^{-12} \mathrm{~m}^{2} / \mathrm{N}$ $5.15 \times 10^{-12} \mathrm{~m}^{2} / \mathrm{N}$

Average birefringence value of the specimen on calculation is $1.26 \times 10^{-5} \mathrm{~nm} / \mathrm{cm}$ whereas birefringence value at the centre of the specimen is $1.22 \times 10^{-5} \mathrm{~nm} / \mathrm{cm}$. The mathematical calculated birefringence value of the specimen is $1.18 \times 10^{-}$ ${ }^{5} \mathrm{~nm} / \mathrm{cm}$ based on photoelastic principle. The photoelastic constant calculated from the calibration procedure is mapped to obtain birefringence values. The birefringence values obtained in the sample from theoretical, experimental and simulation results are in good agreement. Thus, the photoelastic constant of the specimen from the calibration procedure will be implemented for microfluidic channels to obtain birefringence values. The calculated stress optical coefficient (C) is $5.14 \times 10^{-12} \mathrm{~m}^{2} / \mathrm{N}$ which is almost equivalent to the generic stress optical coefficient value (C) of PMMA.

\subsubsection{COC (Cyclic Olefin Co-polymer)}

As we already established a procedure to obtain birefringence values from phase difference values via calibration technique, we implemented the same procedure 
to obtain birefringence values of COC rheological sample at different loads as shown in Figure 4.12. The obtained birefringence includes intrinsic birefringence induced during manufacturing because of the thermal gradient, stress gradient and ambient factors. Thus, to compensate or to get rid of intrinsic birefringence, the birefringence value of the sample at no-load state $\left(\mathrm{B}_{0}\right)$ is calculated. Further, the birefringence obtained at different loads $\left(\mathrm{B}_{\mathrm{L}}\right)$ is compensated with its intrinsic birefringence values. The birefringence value is plotted along the centre to observe its common characteristic for varying load values. The intrinsic retardation values in the material are mapped to birefringence values by the aid of the photoelastic constant.
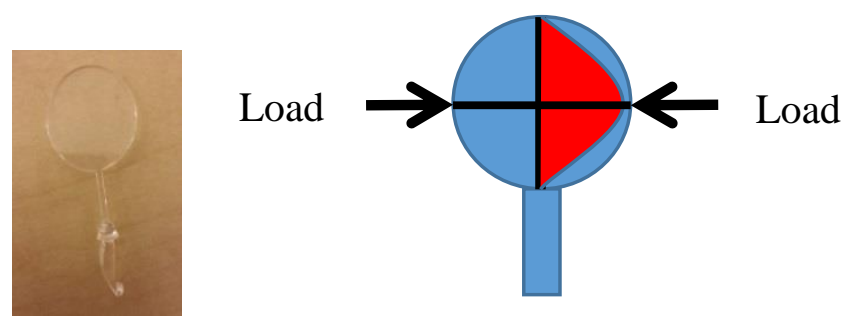

Figure 4.12: Circular protruded acrylic specimen (left), schematic diagram (right) Figure 4.13 depicts the stress induced birefringence present in the specimen at various loads. The load was applied through load cell and the images were captured and processed to compute the desired results. The induced birefringence value was calculated and plotted along a vertical diameter with and without residual stress. The intrinsic birefringence values were subtracted from the stress induced birefringence values to get actual birefringence on increased loading. Hence, we plotted the effect of load on centre line as in Figure 4.14 and Figure 4.15 with and without intrinsic birefringence respectively. Further, we calculated 
the photoelastic constant [93-99] of the material in increasing load for verification of our system.

Here, fringe constant $f_{\sigma}=\frac{\lambda}{C}$. Since we calculate the fringe constant by calibration procedure; we can assess the material photoelastic constant value (C).

$0 \mathrm{~kg}$

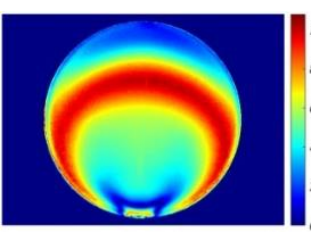

$3 \mathrm{~kg}$

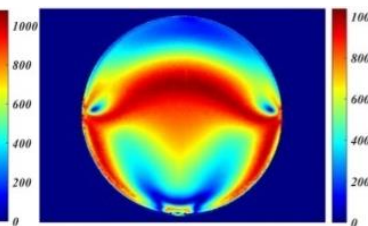

$4 \mathrm{~kg}$

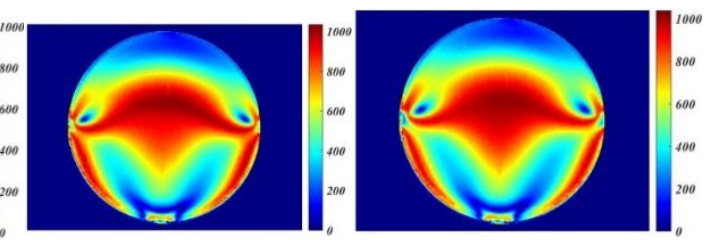

Figure 4.13: Induced stress birefringence for cyclic olefin copolymer at increasing loads

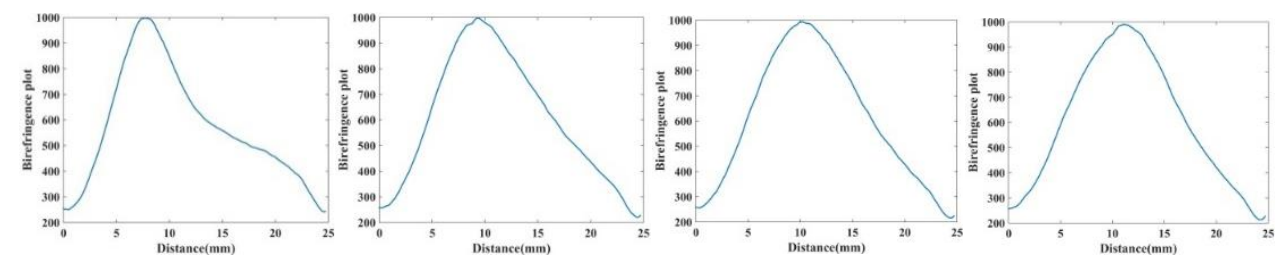

Figure 4.14: Birefringence induced in specimen along vertical diameter (with residual stress)

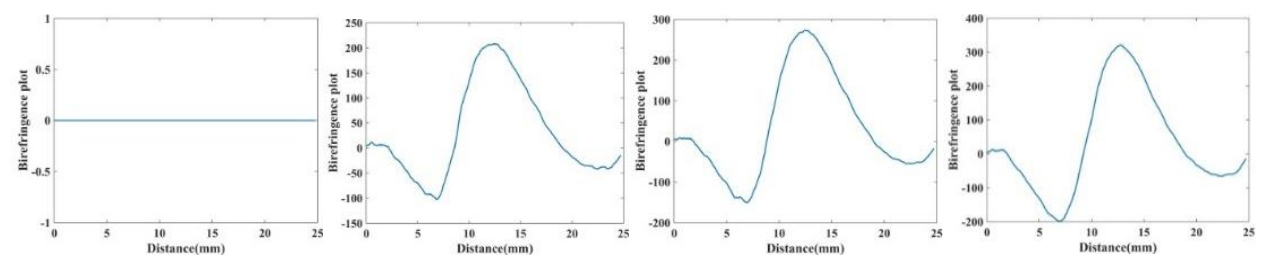

Figure 4.15: Birefringence induced in specimen along vertical diameter (without residual stress)

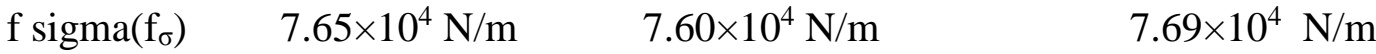

Material Fringe Constant (C) $7.19 \times 10^{-12} \mathrm{~m}^{2} / \mathrm{N}, 7.24 \times 10^{-12} \mathrm{~m}^{2} / \mathrm{N} \quad 7.15 \times 10^{-12} \mathrm{~m}^{2} / \mathrm{N}$

The photoelastic constant $(\mathrm{C})$ of the material is in good agreement with the provided COC photoelastic constant values[24]. The material fringe constant and 
photoelastic constant of the sample were calculated for verification of our system. This measurement is vital for further assessment of birefringence values in microfluidic channels.

\subsection{Microfluidic channels}

Microfluidic channels have tremendous application in modern medicine and scientific research laboratories. Microfluidic channels are manufactured through injection moulded technique. Injection moulded technique is predominant due to its cheap manufacturing cost and mass production rate. Microfluidic channels are deemed to be precise in shape, size and form. Stress-induced distortion, temperature gradient and free mould shrinkage are the underlying problems for precise manufacturing. It causes an optical aberration in the samples hence difficult to get the absolute measurement values. The most commonly used microfluidic channels are fabricated from cyclic olefin copolymer (COC) and poly-methyl-methacrylate (PMMA) materials. So, we chose COC and PMMA as a calibration sample to obtain the photoelastic constant of the material as described in the Section 4.6.1. The circular specimen is used for calibration purpose and obtained photoelastic constant values from the samples are mapped to microfluidic channels. The qualitative and quantitative measurement values of birefringence is determined through birefringence measurement system. The obtained photoelastic constant value for the PMMA and COC is $5.14 \times 10^{-12} \mathrm{~m}^{2} / \mathrm{N}$ and $7.19 \times 10^{-12} \mathrm{~m}^{2} / \mathrm{N}$. The obtained photoelastic constant values are mapped into residual stress values to birefringence values. The birefringence values are qualitatively and quantitatively inspected for sample inspection and product verification. This sample inspection will lead to select microfluidic channels of 
desired properties. Microfluidic channels have huge application in biological labs and research institutes, thus minute and detailed measurement is essential. The biological inspection and measurements are delicate and sensitive imperative for biological diagnosis and follow-up. The outcome of this research work provides a suitable approach for measuring birefringence in microfluidic channels.

\subsection{Implementation in microfluidic channel}
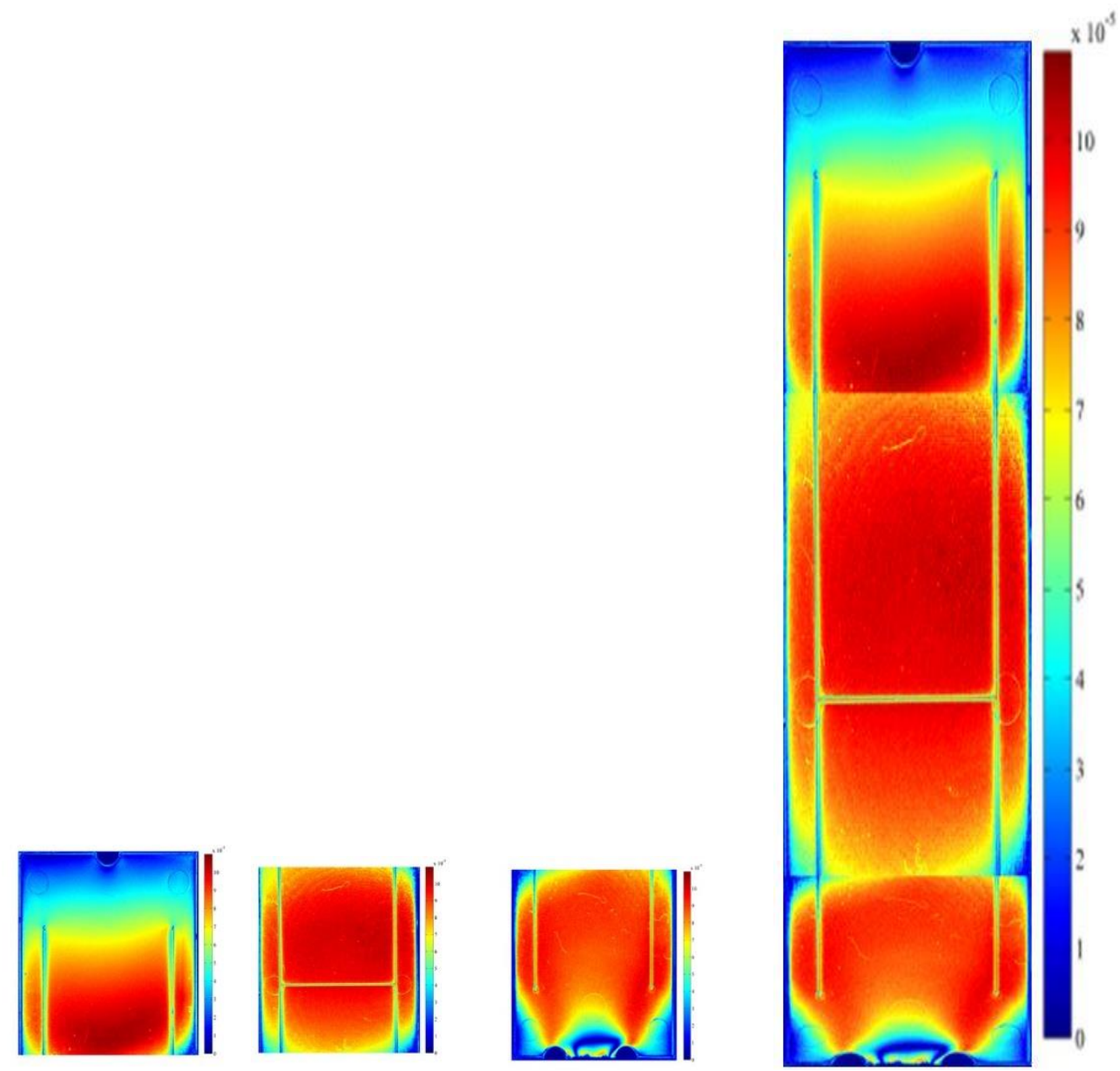

Figure 4.16: Birefringence in microfluidic $(\mathrm{H})$ channel of top, middle and bottom with full part

The average birefringence value of the specimen is found to be $5 \times 10^{-5} \mathrm{~nm} / \mathrm{cm}$. Birefringence value of microfluidic channels is least at the edges whereas maximum at the centre part. The birefringence gradient prevails at the notches that 
can be recognized at two notches i.e. upper and the lower notch of the specimen as shown in Figure 4.16. The channel birefringence profile seems to be of constant magnitude throughout the specimen. From the calibration procedure, the birefringence present in the $\mathrm{H}$ channels is calculated as given below:

$$
n_{1}-n_{2}=C\left(\sigma_{1}-\sigma_{2}\right)
$$

Hence, by mapping residual stress values with stress optical coefficient, we determine the birefringence distribution values throughout the material. This measurement will aid in the qualitative and quantitative selection of microfluidic channels vital for medical and biological research laboratories. Microfluidic channels have been extensively used in the biomedical field and nanotechnology. Disparity or non-uniformity during manufacturing causes birefringence incorporating error in measurement values. This birefringence distribution value is useful for quality testing of the specimen in industrial level and biological research institutes.

\subsection{Effect of Roughness in Birefringence}

We inspect an alteration in induced birefringence values on increasing roughness in the specular sample particularly microfluidic channels. The sample for the microfluidic channel is an acrylic material (Poly-methyl Methacrylate) with a diameter of $17.6 \mathrm{~mm}$ and a thickness of $3.0 \mathrm{~mm}$. The roughness is generated on the sample by grinding against $\mathrm{SiC}$ (Silicon carbide) paper of different grit size. The SiC paper of different grit size is shown in the Figure 4.17. The highest number in $\mathrm{SiC}$ indicates finer (smaller) grit size whereas lowest number has coarse (larger) grit size which imparts roughness of higher values. So, we started 
grinding our acrylic sample with a finer grit size and gradually increased to coarse grit size which simultaneously increases the roughness of the sample. The roughness and the birefringence values of the sample are measured after each successive grinding. Average, maximum and minimum roughness values are computed for the sample after each grinding steps of different grit size. Similarly, average, highest and lowest birefringence values are computed with an increase in roughness values after each grinding steps.

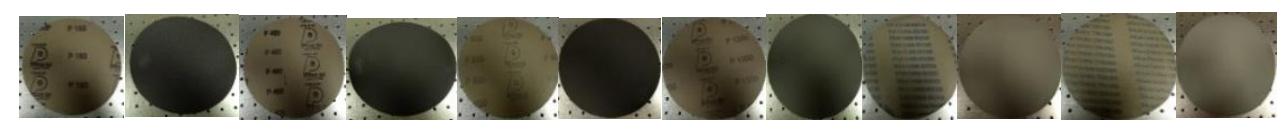

Figure 4.17: SiC paper of different grit size (P180, P400, P800, P1200, P2400, P4000)

Roughness values on the samples are measured by Talysurf profilometer Talyscan 150. The Talyscan profilometer has a vertical resolution of $0.06 \mu \mathrm{m}(60 \mathrm{~nm})$ and with a measurement range of $2.5 \mathrm{~mm}$.

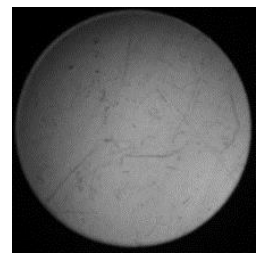

Figure 4.18: Intrinsic roughness of the PMMA sample

Sample specimen and its inherent roughness is represented in the Figure 4.18. Sample specimen roughness and birefringence values of PMMA (polymethyl methacrylate) after each successive grinding is calculated. Different grit size generates roughness in micrometer $(\mu \mathrm{m})$ alongwith birefringence values $(\mathrm{nm})$ throughout the sample. The obtained birefringence values is normalised with the thickness and obtained in $\mathrm{nm} / \mathrm{cm}$ values. Furthermore, mean roughness of the 
sample is computed and compared with mean birefringence values after each successive grinding. The roughness generated in the sample after each successive grinding from different grit size is presented in the Figure 4.19.
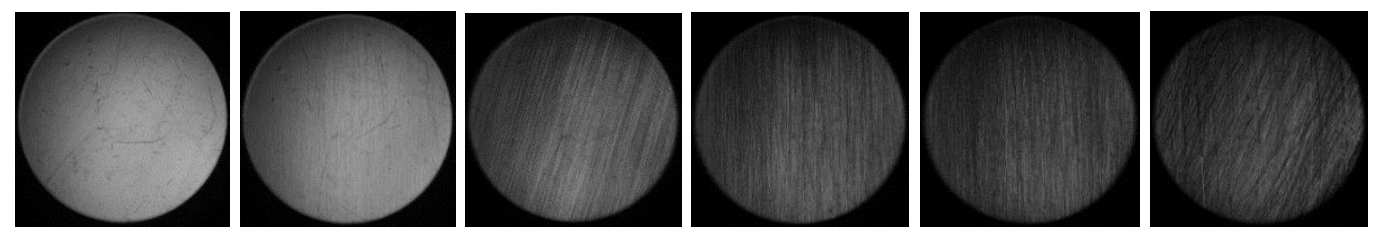

Figure 4.19: Roughness on sample from different grit size (i.e. P4000, P2400, P1200, P800, P400, P180)

The intrinsic birefringence value of the sample is computed for further comparison which is shown in Figure 4.20.

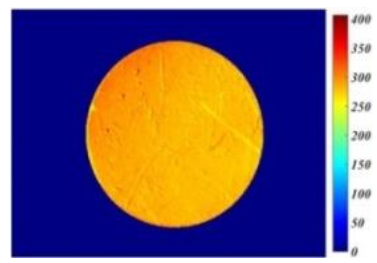

Figure 4.20: Intrinsic Birefringence $(\mathrm{nm} / \mathrm{cm})$ values of the PMMA sample

In addition to that, the birefringence of the sample is computed after each grinding steps of different grit size. Figure 4.21 represents the birefringence values of the sample calculated after each grinding steps of different grit size.

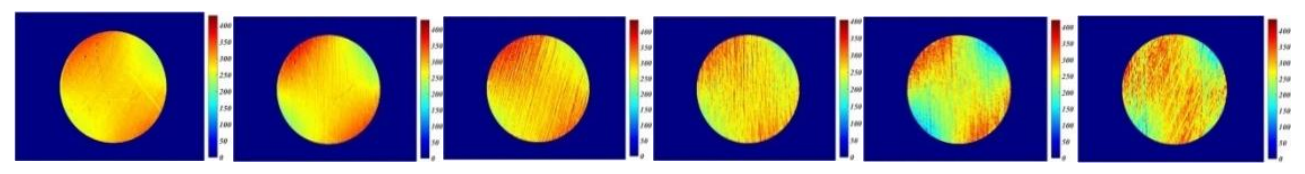

Figure 4.21: Birefringence $(\mathrm{nm} / \mathrm{cm})$ observed on sample by different grit size (P4000, P2400, P1200, P800, P400 and P180) 
To assess the roughness in the sample after successive grinding, we plot the roughness profile along the vertical diameter of the sample. The roughness profile of the sample generated from different grit size is measured along the vertical diameter in micrometre unit is represented from Figure 4.22 to Figure 4.29.

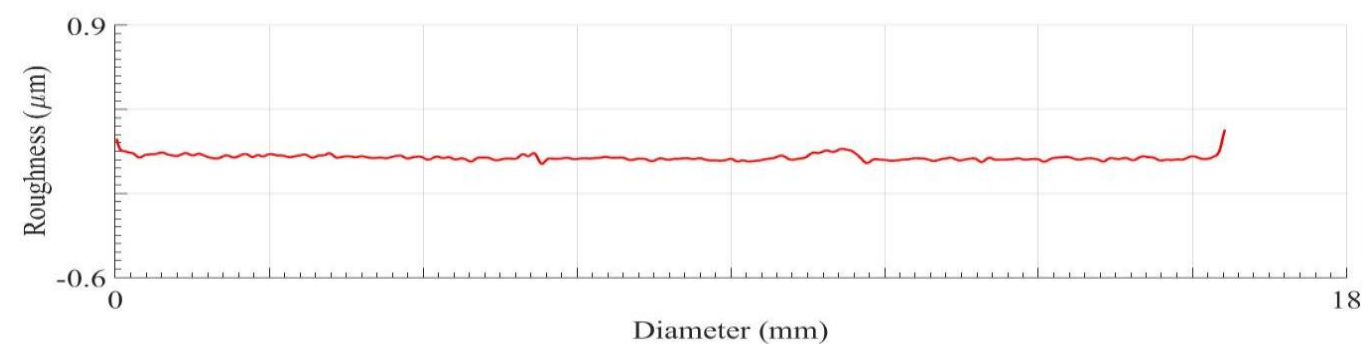

Figure 4.22: Roughness of the original specular sample along vertical diameter

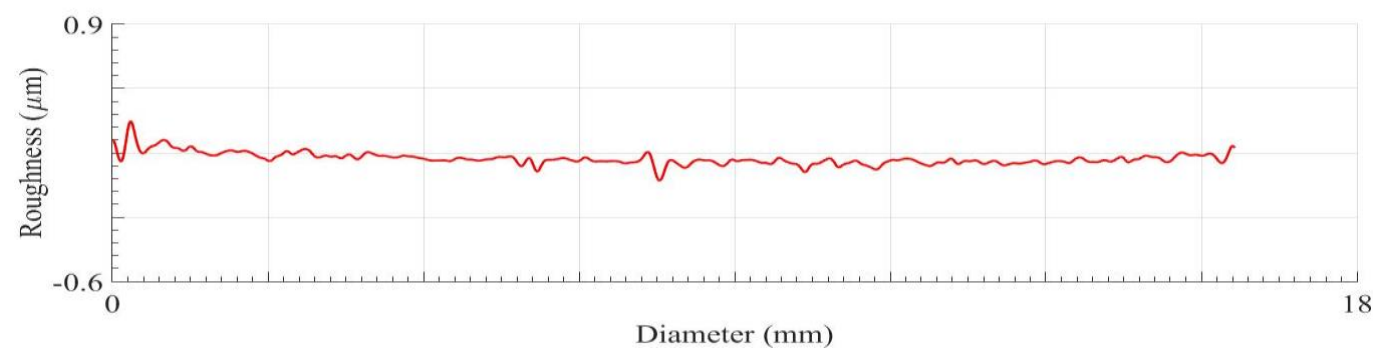

Figure 4.23: Roughness of the sample after $\mathrm{P} 4000$ grit size grinding along vertical diameter

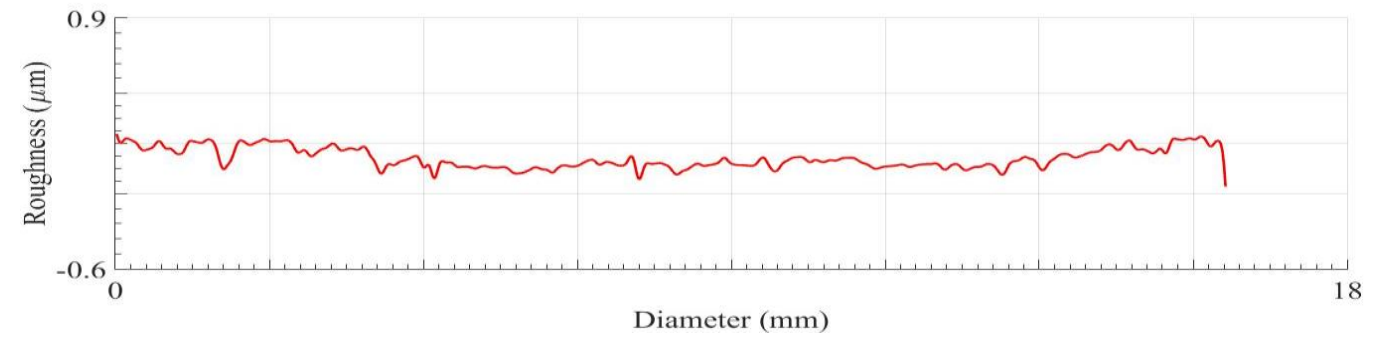

Figure 4.24: Roughness of the sample after P2400 grit size grinding along vertical diameter 


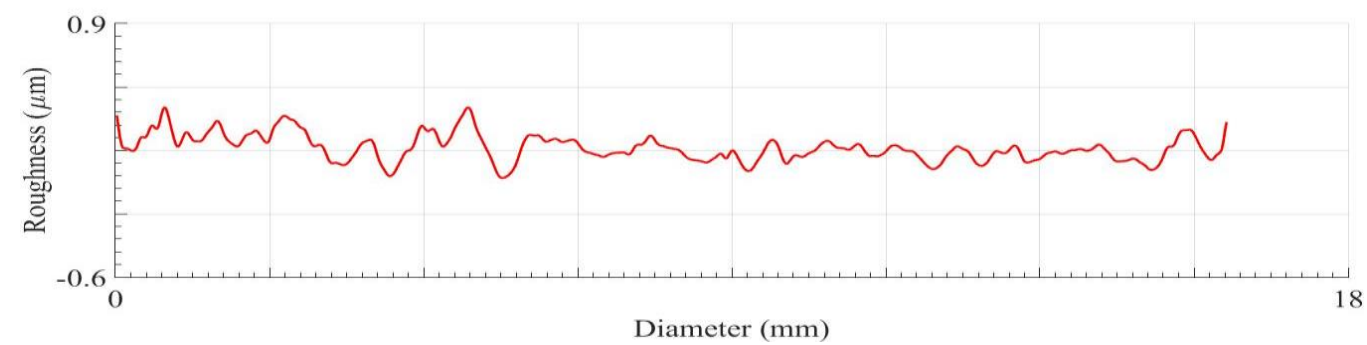

Figure 4.25: Roughness of the sample after P1200 grit size grinding along vertical diameter

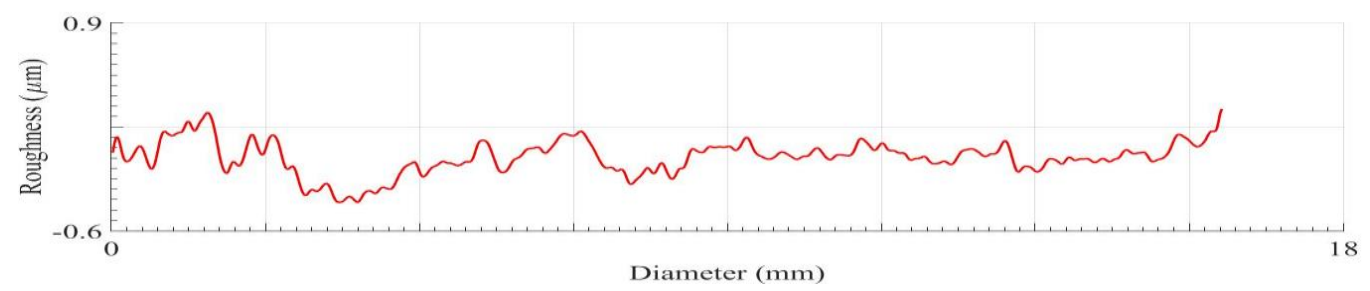

Figure 4.26: Roughness of the sample after P800 grit size grinding along vertical diameter

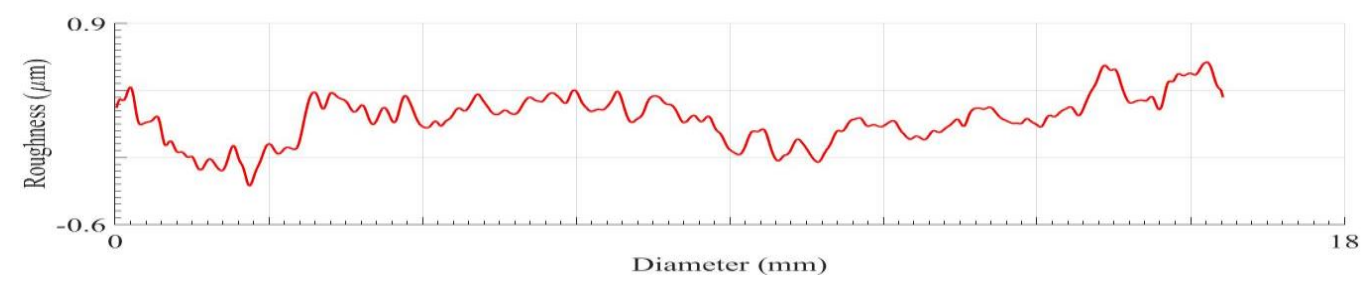

Figure 4.27: Roughness of the sample after $\mathrm{P} 400$ grit size grinding along vertical diameter

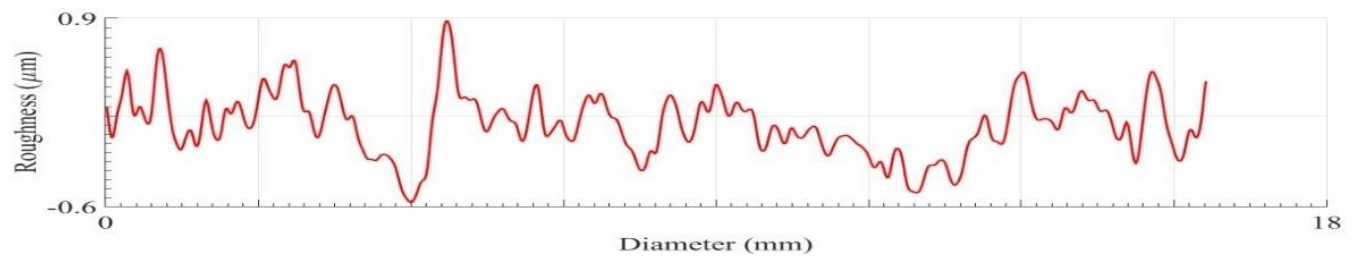

Figure 4.28: Roughness of the sample after P180 grit size grinding along vertical diameter

Roughness and birefringence values of the sample are computed and well tabulated as shown in 
Table 4.5. From the tables and figures, we can infer that there is an increase in birefringence values with increase in roughness of the sample.

\begin{tabular}{|c|c|c|c|}
\hline Sample & $\mathrm{SiC}$ grit size & Roughness $\left(R_{q}\right)$ in $\mu \mathrm{m}$ & Birefringence $(\mathrm{nm} / \mathrm{cm})$ \\
\hline \multirow{7}{*}{ 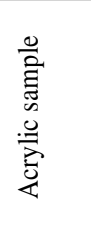 } & Reference & 0.07 & 147.56 \\
\hline & $\mathrm{P} 4000$ & 0.09 & 198.23 \\
\hline & P2400 & 0.11 & 200.19 \\
\hline & P1200 & 0.14 & 208.21 \\
\hline & P800 & 0.18 & 221.26 \\
\hline & $\mathrm{P} 400$ & 0.23 & 250.43 \\
\hline & P180 & 0.61 & 277.91 \\
\hline
\end{tabular}

Table 4.5: Birefringence observed in the sample on increasing Roughness $\left(R_{q}\right)$

There seems to be an approximately linear relationship between the increase in birefringence with an increase in specimen roughness values as shown in the Figure 4.29. Although the relationship is not completely linear, there are other factors that come into play. Stress, temperature gradient and other ambient factors along with material intrinsic property is the reason behind nonlinear increase in birefringence values with increase in roughness.

\section{Birefringence due to Roughness}

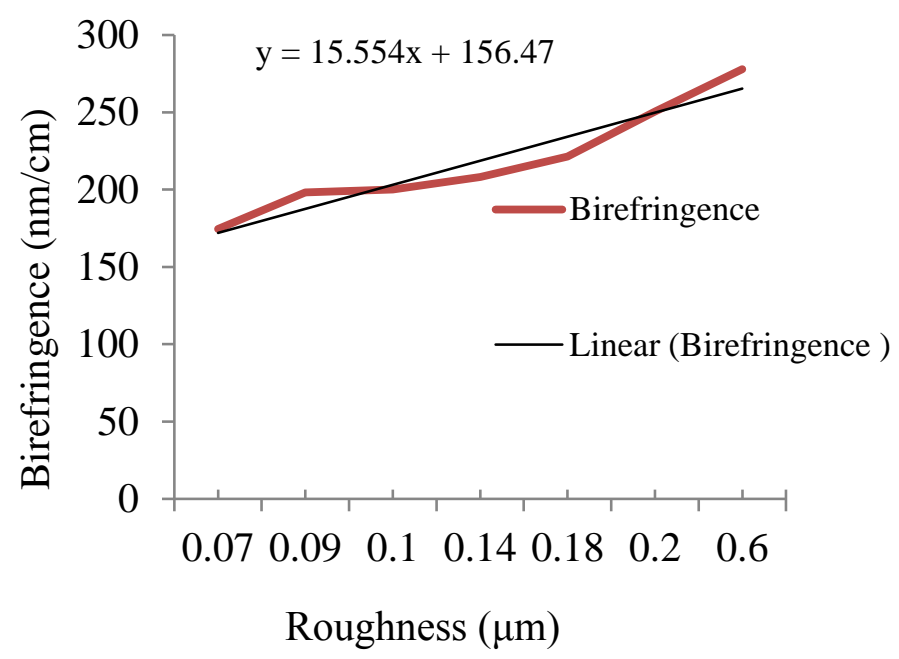

Figure 4.29: Relationship between birefringence effects on increasing roughness of the sample 


\subsection{Birefringence measurement of optical windows}

It is a piece of transparent optical material that allows transmitting light of a certain wavelength and bandwidth. Optical windows facilitate the clear aperture for fixed wavelength of light and restrict other wavelength of light. Its usage ranges from the protection of optical instruments against hostile natural environments to the contaminants of exceptional man-made environments. Optical windows are designed to be precise with improved functionality and less noise, especially in crucial and advanced applications. Advanced optical windows are excellent performers when it comes to aerospace, military, commercial avionics, scientific, medical, academic, research and industrial applications. These are generally manufactured from two flat polished surfaces with fine transparency. They are functioned for sealing, protecting without disturbing transparency of substrate material. Optical windows should be selected based on the material transmission or mechanical properties of the substrate. Optical windows should not alter the magnification of a system. It has tremendous application in manufacturing industries, biomedical research institutes and military defence.

\subsubsection{Part diagram and specification}

Birefringence measurement system for optical windows comprises of following features as shown in Table 4.6.

\begin{tabular}{|cr|}
\hline \multicolumn{1}{|c|}{ Specifications } \\
\hline Retardation resolution & $1.0 \mathrm{~nm}$ \\
\hline Wavelength & $658 \mathrm{~nm}$ \\
\hline Measurement speed & 5 seconds \\
\hline Working distance & $14 \mathrm{~cm}$ \\
\hline Stage travel $(\mathrm{x}, \mathrm{y})$ & $10 \mathrm{~cm}, 10 \mathrm{~cm}$ \\
\hline Field of View & $\mathbf{( 5 0} \mathbf{~ m m} \times \mathbf{5 0} \mathbf{~ m m})$ \\
\hline
\end{tabular}

Table 4.6: Specification of the measurement system 


\subsubsection{Offset Matrix algorithm}

We should measure the intrinsic birefringence in the red filters as shown in Figure 4.30. To measure this sample, we require specific quarter wave plate for red wavelength. Since our system has quarter wave plate for green colour wavelength, we are trying to measure it by our existing system that possess quarter wave plate for green colour. To resolve this problem, we calculate the deviation in phase difference values due to mismatch in quarter wave plate for the specific wavelength. Later, the phase difference values are compensated. In this experiment, the light source opted is of red colour wavelength $(658 \mathrm{~nm})$ while quarter wave plate is appropriate for green colour wavelength $(532 \mathrm{~nm})$. Here, we are trying to compensate the offset phase difference mathematically due to mismatch in quarter wave plate and calculate the real phase difference values. The light from low birefringence polariscope will not be perfectly circularly polarised and the phase values are deviated because of wavelength dependence[100] of light. Thus, we find the offset values mathematically and compensate on the obtained phase values to compute the real phase values as in equation (4.13) by,

$$
\epsilon=\frac{\pi}{2}\left(\frac{\lambda_{m}}{\lambda}-1\right)
$$

Here, matching light source wavelength for designed quarter waveplate is represented by $\lambda_{m}$ (i.e. $\lambda_{m}=532 \mathrm{~nm}$ ) while light source wavelength employed is represented by $\lambda(\lambda=658 \mathrm{~nm})$. Thus, error $(\epsilon)$ on phase difference values were calculated theoretically and experimentally and is in good agreement. Compensated phase values were mapped to birefringence values. The computed 
birefringence values of the red filters are compared to the standard values manufactured by the company and measured results are in good agreement.

\subsection{Experiments and results}
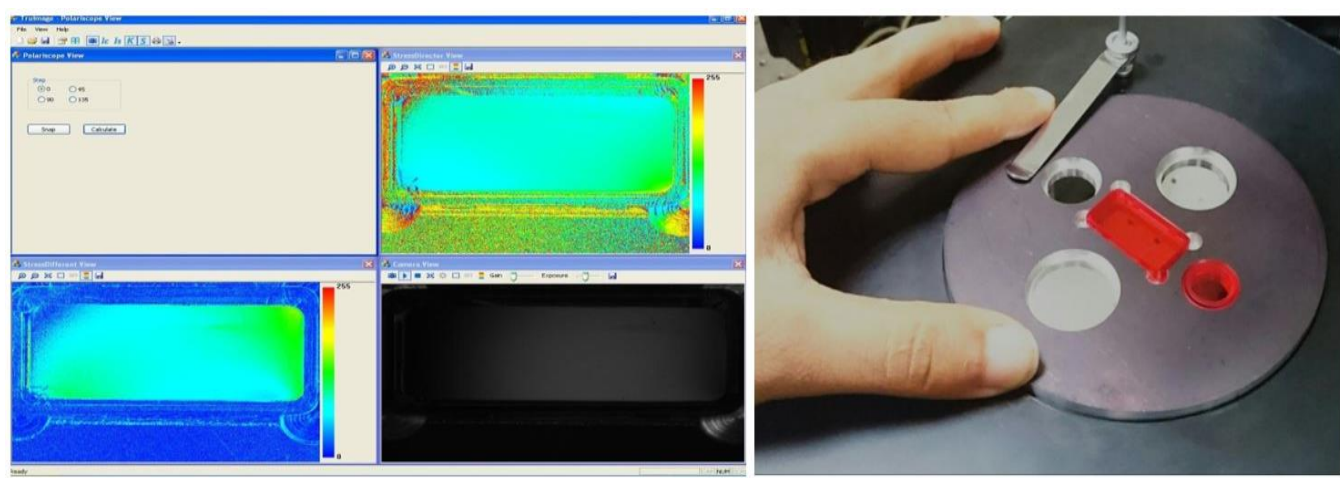

Figure 4.30: Setup for the measurement of two optical windows of different shape in polariscope

There are fourty red filters, twenty of circular type and twenty of rectangular type. The samples are made up of PMMA (Delpete 80n type) as shown in Figure 4.30. It has high heat resistance and strength. It is highly recommended for taillight lenses, reflectors and other auto parts. The sample provided is optical windows as a sensor for conveyors. Delpete $80 \mathrm{n}$ are widely used because of its fine transparency, elegant lustre, colour brightness, excellent weather resistance, surface hardness, excellent chemical resistance, superior electrical resistance, insulation properties and superior machinability.

\subsubsection{Transparent windows}

We selected transparent sample as shown in Figure 4.31 with the region of interest $(6.5 \mathrm{~mm} \times 6.5 \mathrm{~mm})$ to inspect its intrinsic birefringence values for further evaluation. The mean birefringence value of the sample is $566.18 \mathrm{~nm} / \mathrm{cm}$. The measured values were in accord with their standard values. 


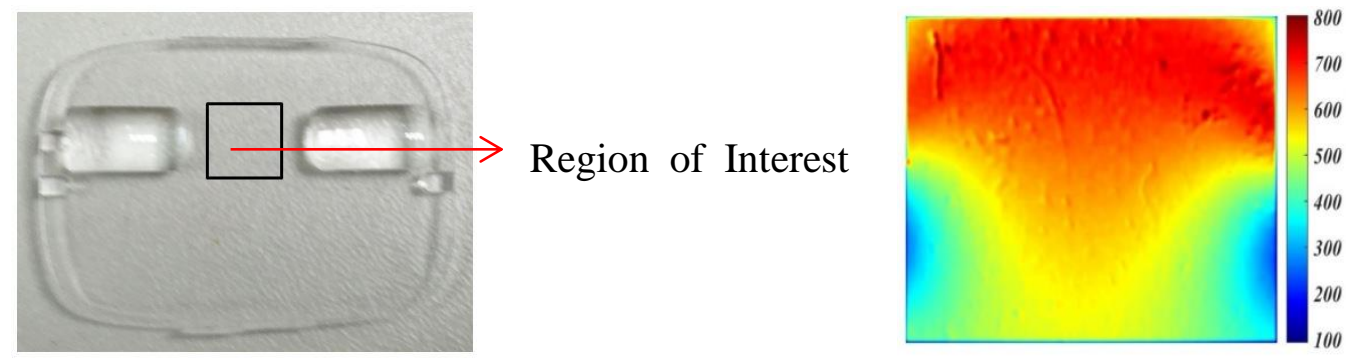

Figure 4.31: Left (Transparent window sample), Right (Birefringence Image)

\subsubsection{Circular samples result}

The sample is a red filter of external diameter $15 \mathrm{~mm}$ and an internal diameter 12 $\mathrm{mm}$ as shown in Figure 4.32 and its thickness of $1.5 \mathrm{~mm}$. The mean birefringence value of the sample is $22.18 \mathrm{~nm} / \mathrm{cm}$.

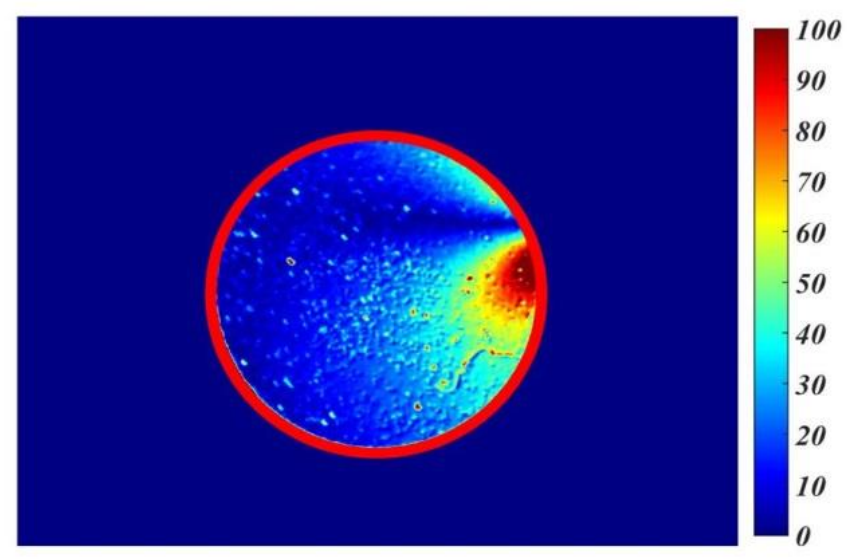

Figure 4.32: Birefringence values in $\mathrm{nm} / \mathrm{cm}$ of circular window filter

The measured values of birefringence of the samples by our system are in good agreement with company measured values. Thus, birefringence measurement of optical windows helps in material selection by rejecting defective parts. Further, birefringence values of the rest of the circular samples are provided in the Appendix. 


\subsubsection{Rectangular samples results}

The red rectangular samples are used as optical filters as belt sensors. It has a length of $30.00 \mathrm{~mm}$, breadth of $16.00 \mathrm{~mm}$ and thickness of $1.00 \mathrm{~mm}$ as shown in Figure 4.33.

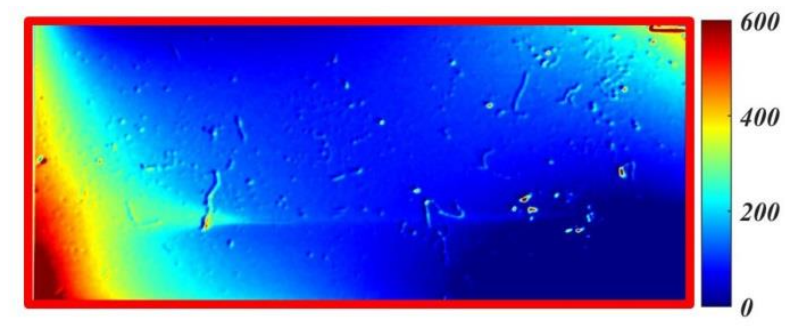

Figure 4.33: Birefringence values in $\mathrm{nm} / \mathrm{cm}$ of rectangular window filter

The average birefringence value of the rectangular sample is $123.76 \mathrm{~nm} / \mathrm{cm}$. The average birefringence values of the rectangular samples are in good agreement with standard values provided by the company. Localised birefringence values at localised area might not represent the whole specimen. Average birefringence values were computed for material selection. Since the material is supposed to have constant birefringence, average birefringence values are highly recommended. Further, birefringence values of the rest of the rectangular samples are provided in the appendix.

\subsection{Comparison of Research method algorithm with commercial}

\section{method}

The material constant value is computed from the calibration procedure as mentioned in Section 4.6. This material constant value is mapped for computation of birefringence values in the material. In this experiment, we compare phase difference images at the centre measured by phase shift polariscope and 
commercial measurement system. The respective residual stress values due to the intrinsic phase difference are calculated and plotted along vertical diameter from both commercial Hinds photoelastic modulator (PEM) system and phase shift polariscope (PSP) as shown in Figure 4.34. There is a good correlation of measurement values in both measurement system. Further, error and accuracy of the measurement systems were carried out for further assessment.
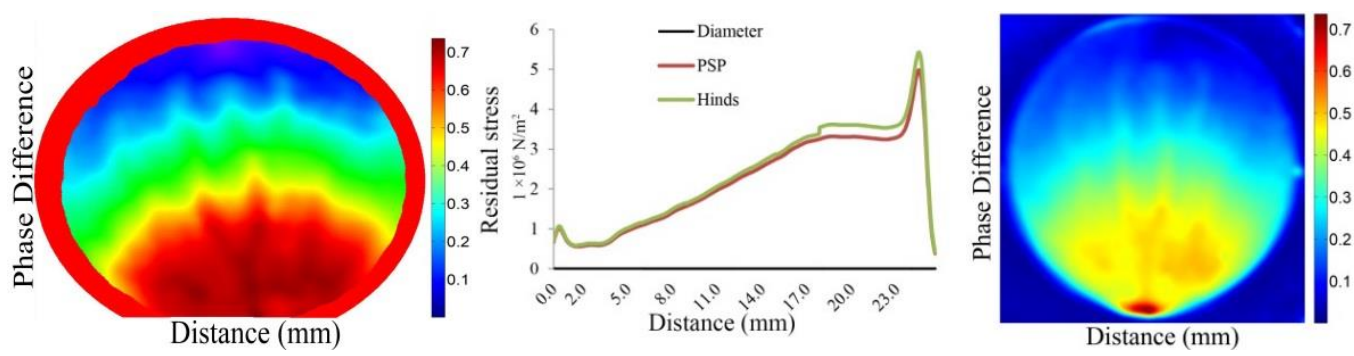

Figure 4.34: Phase difference comparison with Hinds commercial and phase shift method

$$
\begin{aligned}
& \text { Error }=\frac{0.0768 \times 10^{-5}}{2.7924 \times 10^{-5}} \times 100 \% \\
& =2.75 \% \\
& \text { Accuracy }=1 \text {-error }=97.25 \%
\end{aligned}
$$

Hence, the accuracy of the measurement from both system is found to be $97.25 \%$ accurate for PMMA. The commercial system has its own limitations, for instance point to point scanning and low-resolution measurement system. These drawbacks are surpassed by phase shift polariscope with its one shot full field high-resolution measurement. 


\subsection{Summary}

In this work, birefringence measurement of various plastic specimen manufactured by injection moulding is carried out. We conducted an inspection of micro-wells which is a standard tool in analytical research and in diagnostic laboratories. Maximum birefringence is observed in BD Falcon, Grenier and NUNC 384 specimen made up of polystyrene, polyolefin and polypropylene material. Ideally, transparent microplates are stress-free and the refractive index is uniform in nature. Factors specifically stress gradient, thermal gradient limits uniformities during manufacturing of injection moulded samples. Lower and uniform birefringence provides fine luminescence, discernment and highresolution whereas high and non-uniform birefringence makes reading or data values inaccurate. Thus, cyclic olefin copolymers are mostly used because of its low level of auto fluorescence, exceptional high clarity in UV range of low birefringence to that of polypropylene and polystyrene plates. Birefringence values and its distribution measured by our system is in well agreement with a commercial system. This system has been a vital tool for qualitative and quantitative inspection of optical specimens in biological research institutes and diagnostic laboratories.

Rheological samples of PMMA and COC are qualitatively and quantitatively assessed by its birefringence properties. Applied stress induces retardation in the optical specimen also known as stress induced birefringence in the specimen. Maximum birefringence occurs at the centre point during two-point loading subjected to inherent material properties. This study is very crucial to observe how the stress generated during manufacturing engenders birefringence in the material. 
Moreover, we invented procedure for quantifying residual birefringence and its characteristics at varying load. The material photoelastic constant of COC and PMMA is determined and is within the standard acceptable range. This birefringence measurement system continually examines the sample uniformity during manufacturing, i.e. in situ inspection of the manufactured samples. Overall, the system is equipped for rapid non-destructive measurement of stress-induced birefringent optical samples. This measurement is vital for determining specimen optical properties. Therefore, with these salient features, the system is deployed for process and quality control of injection moulded optics.

Similarly, visualisation and quantification of the process induced birefringence in microfluidics is carried out. Birefringence measurement system is single shot whole field measurement system with high resolution. Calibration procedure with similar samples is performed for an injection moulded circular disk in diametric compression. Stress-optical coefficient determined using this method complies with the standard value as provided in injection moulding handbook. This method is useful for assessing the birefringence (anisotropy) of the injection moulded sample like microfluidic channels. Qualitative and quantitative inspection of microfluidics specimen by polariscope is vital in manufacturing companies and R\&D (Research and Development) laboratories. Residual stress and birefringence measurement in microfluidics by polariscope has been significant tool for quality testing and improvement in manufacturing. 


\section{Chapter 5: Low birefringence measurement system}

In this chapter, a completely automated system for measuring birefringence of the optical samples is designed and the measured results are validated by the prevalent commercial system. This system abrogates the tedious manual phase shifting method prone to manual errors[101]. To overcome the manual errors, this system has in-built motorised analyser with an accuracy of $0.01^{0}$, nullifying the errors. In contrast, manual method has problems particularly vibration instability, backlash error which is absent in the automated system. Further, it can be utilised for 3D (three-dimensional) polarisation imaging[20, 102] of the specimen. This system has huge scope in the industry, research and development (R\&D) sector. It can be implemented in IR (Infra-red) bandwidth for identifying cracks and defects in Si (Silicon) wafers[80].

\subsection{Introduction}

It constitutes of multiple elements, i.e. visible light source, polarizers, quarter wave plate and CCD (Charge-coupled Device) camera as shown in Figure 5.1. It has a tuneable light source and is applicable for wavelength dependent material. The light source is LED (Light Emitting Diode) of $50 \mathrm{~mm}$ diameter and can be tuned to visible range from 380 to $740 \mathrm{~nm}$ of distinct colours. The holographic diffuser is kept in between the light source and the polarizer to obtain uniformly collimated beam with maximum beam deviation of $5^{0}$. The spatially uniform light beam is passed through light source, polariser, quarter wave plate, specimen and automated analyser. Automated analyser captures four phase shifted images with high precision and accuracy. The four phase shifted images are captured by 
camera and computed via image processing system to obtain birefringence values of the specimen. The polarizer implemented in the system is within visible range (400 to 800$) \mathrm{nm}$. The quarter wave plate implemented is of $532 \mathrm{~nm}$ designed for the green colour wavelength of light. Light source emission wavelength is of 520 nm which should comply with the quarter wave plate operating wavelength. So, there is minute offset in wavelength of light source and quarter wave plate wavelength. To compensate this offset, compensation equation has been formulated and successfully implemented. The sample stage is translational rotational two axes $(\mathrm{X}, \mathrm{Y})$ stage where we can scan the larger specimens up to 15 $\mathrm{cm} \times 15 \mathrm{~cm}$ dimensions. The analyser is fully automated to obtain the phase shifted images with a high degree of accuracy and precision. The detector camera DMK41AF02 has a large FOV (Field of View) of $50 \mathrm{~mm} \times 50 \mathrm{~mm}$ with a larger pixel size of $4.65 \mu \mathrm{m}$. The sensor is CCD (charge-coupled device) with high quantum efficiency (>70\%) and less noise. The system has been modelled both in Jones and Mueller calculus. More parameters like depolarization[103, 104], transmittance and scattering parameters are exploited by analysing Mueller matrix transformation[105-107] of the entire system. It is applicable for characterising subwavelength metrological structure for identifying various properties [108].

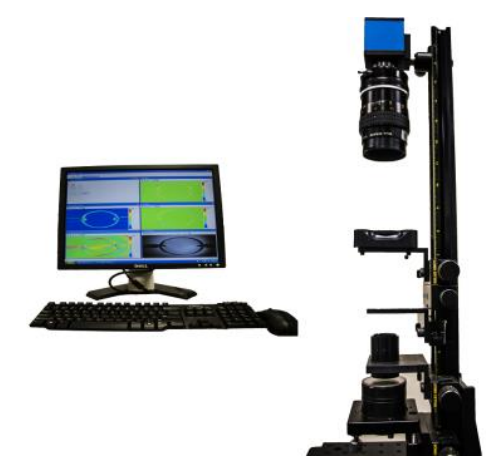

Figure 5.1: Low Birefringence measurement system 


\subsection{Theoretical Background}

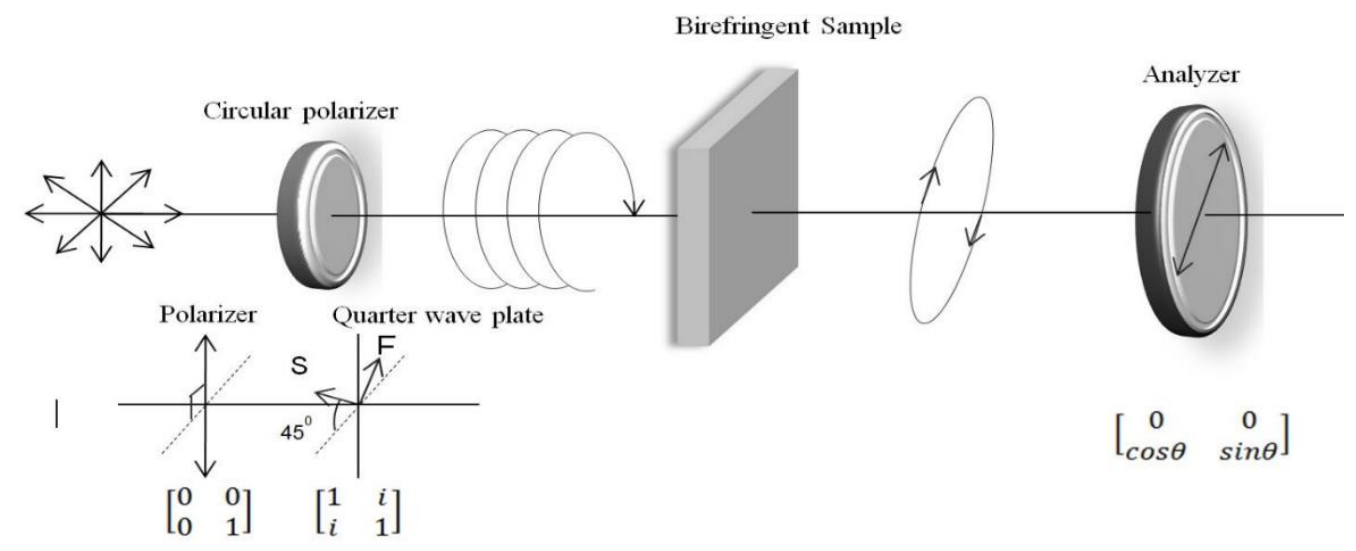

Figure 5.2: Schematic diagram of Low Birefringence Polariscope (LBP)

The setup of low birefringence polariscope is schematically shown in Figure 5.2. Each element is modelled on Jones matrix and vector based on Jones calculus. In addition to that, each element is modelled on Mueller matrix and vector based on Mueller calculus. Mueller matrix modelling and analysis provides additional parameters particularly depolarization, transmittance and retardance.

\subsubsection{Jones calculus in birefringence measurement system}

The light source, polarisers and the quarter wave plate are represented in the form of Jones calculus. Light traverses through polarizer, quarter wave plate, specimen and analyser which is represented by $(\mathrm{U}, \mathrm{V})$ as follows,

$$
\left[\frac{U}{V}\right]=J_{A} J_{M} J_{Q} J_{P} a e^{j w t}
$$

Here $J_{P}, J_{Q}, J_{M}$ and $J_{A}$ indicates Jones vector for polariser, quarter wave plate, material and analyser respectively. On computing the above matrix, we get 


$$
\begin{aligned}
& \left\{\frac{U}{V}\right\}=\frac{\sqrt{2}}{2}\left[\begin{array}{cc}
0 & 0 \\
\cos \alpha & \sin \alpha
\end{array}\right]\left[\begin{array}{cc}
\cos \Delta / 2-i \cos 2 \beta \sin \Delta / 2 & -i \sin 2 \beta \sin \Delta / 2 \\
-i \sin 2 \beta \sin \Delta / 2 & \frac{\cos \Delta}{2}+i \cos 2 \beta \sin \Delta / 2
\end{array}\right]\left[\begin{array}{ll}
1 & i \\
i & 1
\end{array}\right]\left[\begin{array}{l}
0 \\
1
\end{array}\right] a e^{j w t} \\
= & \left.\frac{\sqrt{2}}{2}\left[\left(\sin \frac{\Delta}{2} \cos (\alpha-2 \beta)+\sin \alpha \cos \frac{\Delta}{2}\right)+i\left(\sin \frac{\Delta}{2} \sin (\alpha-2 \beta)+\cos \alpha \cos \frac{\Delta}{2}\right)\right)\right] a e^{j \omega}
\end{aligned}
$$

Here $\alpha$ is phase shift of analyser, $\Delta$ is phase difference of birefringent sample, $\beta$ is inclination of sample towards fast axis, $a$ is the amplitude and $w$ is angular speed of the circularly polarised light.

Light intensity leaving from a specimen is given by,

$$
I=|V|^{2}=\frac{a^{2}}{2}(1+\sin \Delta \sin 2(\alpha-\beta))
$$

Intensity collected at the detector is,

$$
I=I_{a}+I_{c \alpha} \cos 2 \alpha+I_{s \alpha} \sin 2 \alpha
$$

Where,

$$
I_{a}=\frac{a^{2}}{2}
$$

$$
\begin{aligned}
& I_{c \alpha}=-\left(\frac{a^{2}}{2} \sin \Delta\right) \sin 2 \beta \\
& I_{s \alpha}=\left(\frac{a^{2}}{2} \sin \Delta\right) \cos 2 \beta
\end{aligned}
$$

$I_{a}$ is average light intensity, $I_{c \alpha}$ and $I_{s \alpha}$ are cosine and sine component of light intensity received by the detector. 


\subsubsection{Four-step phase shifting method}

In phase shifting photoelasticity, the intensity at four distinct phase is recorded and processed to determine principal stress and direction as shown in Table 5.1. Average light intensity collected is $I_{a}$ which is constant throughout the experiment, four-step phase shifting approach is applied to determine unknowns $\Delta$ and $\beta$. Four step phase shifting and its image intensity with its corresponding equations are shown below:

\begin{tabular}{|r|r|r|}
\hline Steps & Analyzer orientation $(\boldsymbol{\alpha})$ & Intensity equation \\
\hline 1 & 0 & $\mathrm{I}_{1}=\mathrm{I}_{\mathrm{a}}(1-\sin \Delta \sin 2 \beta)$ \\
\hline 2 & $\pi / 4$ & $\mathrm{I}_{2}=\mathrm{I}_{\mathrm{a}}(1+\sin \Delta \cos 2 \beta)$ \\
\hline 3 & $\pi / 2$ & $\mathrm{I}_{3}=\mathrm{I}_{\mathrm{a}}(1+\sin \Delta \sin 2 \beta)$ \\
\hline 4 & $3 \pi / 4$ & $\mathrm{I}_{4}=\mathrm{I}_{\mathrm{a}}(1-\sin \Delta \cos 2 \beta)$ \\
\hline
\end{tabular}

Table 5.1: Phase shifting in low birefringence polariscope

Retardation magnitude and direction are deduced as,

$$
\begin{aligned}
& \Delta=\frac{1}{2 I_{a}}\left(\left(I_{3}-I_{1}\right)^{2}+\left(I_{3}-I_{1}\right)^{2}\right)^{1 / 2} \\
& \beta=\frac{1}{2} \tan ^{-1}\left(\frac{I_{1}-I_{3}}{I_{4}-I_{2}}\right)
\end{aligned}
$$

Hence, retardation magnitude and direction of the principal stress is measured which are further mapped to attain the birefringence values. To map phase difference values to birefringence values, first we should calculate the photoelastic constant of the material. It is obtained through calibration procedure as in Section 4.6. 


\subsubsection{Mueller calculus in birefringence measurement system}

This setup includes the same elements as in birefringence measurement system but modelled via Mueller calculus. The light leaving from the analyser represented by Stokes vector is given by,

$$
S^{\prime}=A_{1} R_{0}(\delta) Q_{45} P_{0} S_{0}
$$

Where $R_{0}(\delta)$ and $Q_{ \pm 45}$ are the Mueller matrices of the specimen and the quarter wave plate whose fast axis is oriented at an angle of $\pm 45^{\circ}$ with the $O x$ axis. Matrices $\boldsymbol{R}_{\mathbf{0}}(\boldsymbol{\delta})$ and $Q_{ \pm 45}$ are obtained on calculating at $\beta=0$ and $\beta= \pm 45^{0}, \quad \delta=$ $90^{0}$, are

$$
\begin{aligned}
& \text { Light source represented by Stokes vector }\left(\mathrm{S}_{0}\right)=\left[\begin{array}{l}
1 \\
0 \\
0 \\
0
\end{array}\right] \\
& \text { Vertical linear polarizer }\left(\mathrm{P}_{0}\right)=\frac{1}{2}\left[\begin{array}{cccc}
1 & -1 & 0 & 0 \\
-1 & 1 & 0 & 0 \\
0 & 0 & 0 & 0 \\
0 & 0 & 0 & 0
\end{array}\right] \\
& \text { Quarter wave plate }\left(\mathrm{Q}_{45}\right)=\frac{1}{2}\left[\begin{array}{cccc}
1 & 0 & 0 & -1 \\
0 & 0 & 0 & 0 \\
0 & 0 & 0 & 0 \\
-1 & 0 & 0 & 1
\end{array}\right]
\end{aligned}
$$

Sample specimen in the system is represented in Mueller matrices by,

$$
R_{0}(\delta)=\left[\begin{array}{cccc}
1 & 0 & 0 & 0 \\
0 & \cos ^{2} 2 \beta+\sin ^{2} 2 \beta \cos \delta & (1-\cos \delta) \sin 2 \beta \cos 2 \beta & -\sin 2 \beta \sin \delta \\
0 & (1-\cos \delta) \sin 2 \beta \cos 2 \beta & \sin ^{2} 2 \beta+\cos ^{2} 2 \beta \cos \delta & \cos 2 \beta \sin \delta \\
-1 & \sin 2 \beta \sin \delta & -\cos 2 \beta \sin \delta & \cos \delta
\end{array}\right]
$$


Similarly, analyser is represented in Mueller matrices by,

$$
\text { Analyser }\left(\mathrm{A}_{1}\right)=\frac{1}{2}\left[\begin{array}{cccc}
1 & \cos 2 \theta & \sin 2 \theta & 0 \\
\cos 2 \theta & \cos ^{2} 2 \theta & \sin 2 \theta \cos 2 \theta & 0 \\
\sin 2 \theta & \sin 2 \theta \cos 2 \theta & \sin ^{2} 2 \theta & 0 \\
-1 & 0 & 0 & 0
\end{array}\right]
$$

The light leaving from the analyser is represented by Stokes vector $S$ ' on polariscope in accordance with Mueller calculus is given by,

$$
S^{\prime}=P_{0} R_{0}(\delta) Q_{45} P_{0} S
$$

On computing the matrix multiplication via MATLAB, we obtain,

$$
S^{\prime}=\left[\begin{array}{c}
1+\cos 2 \theta \sin 2 \beta \sin \delta-\sin 2 \theta \cos 2 \beta \sin \delta \\
\cos 2 \theta+\cos ^{2} 2 \theta \sin 2 \beta \sin \delta-\cos 2 \theta \sin 2 \theta \cos 2 \beta \sin \delta \\
\sin 2 \theta-\cos 2 \beta \sin ^{2} 2 \theta \sin \delta+\cos 2 \theta \sin 2 \theta \sin 2 \beta \sin \delta \\
0
\end{array}\right]
$$

The intensity of light represented by the first term of Stokes vector is given by,

$$
I=1+\cos 2 \theta \sin 2 \beta \sin \delta-\sin 2 \theta \cos 2 \beta \sin \delta=1+\sin \delta \sin 2(\beta-\theta)
$$

The obtained equation is similar to the equation obtained by the Jones calculus modulated at different phase by phase stepping method at $0^{\circ}, 45^{\circ}, 90^{\circ}$ and $135^{\circ}$ to obtain phase retardation and direction values. Thus, phase retardation and direction of the specimen is given by,

$$
\begin{array}{r}
\delta=\sin ^{-1} \frac{\sqrt{\left(I_{3}-I_{1}\right)^{2}+\left(I_{4-} I_{2}\right)^{2}}}{2} \\
\beta=\frac{1}{2} \tan ^{-1}\left(\frac{I_{1-I_{3}}}{I_{4-} I_{2}}\right)
\end{array}
$$


The phase retardation and direction are formulated based on both Mueller and Jones calculus. The system is validated both by Jones and Mueller calculus with same phase difference and direction equations.

\subsection{System Components}

It includes LED (Light Emitting Diode) light arrays in-built on Arduino microprocessor and can be modulated at different wavelengths and intensity level. The rainbowduinoV3.0b is deployed as a light source manufactured by seed studio with operating frequency of $30 \mathrm{~Hz}$ compliant with detectors framerate. It is USB (Universal Serial Bus) manipulated 64 LED arrangements which is portable and can be easily plugged into any notebook for forthright operation. The holographic diffuser of $50 \mathrm{~mm}$ diameter and $5^{0}$ deviations manufactured by Edmund Optics is kept above light source to obtain nearly uniform collimated light. The polarizer of Thorlabs of 2 -inch diameter with transmission ratio more than $80 \%$ and extinction ratio of 1000:1 is shown in Figure 5.3. The extinction ratio is the ratio of maximum transmission of a linearly polarised light when the polarizer axis is aligned with its axis to the minimum transmission of a linearly polarised light when the polarizer is rotated by $90^{\circ}$.

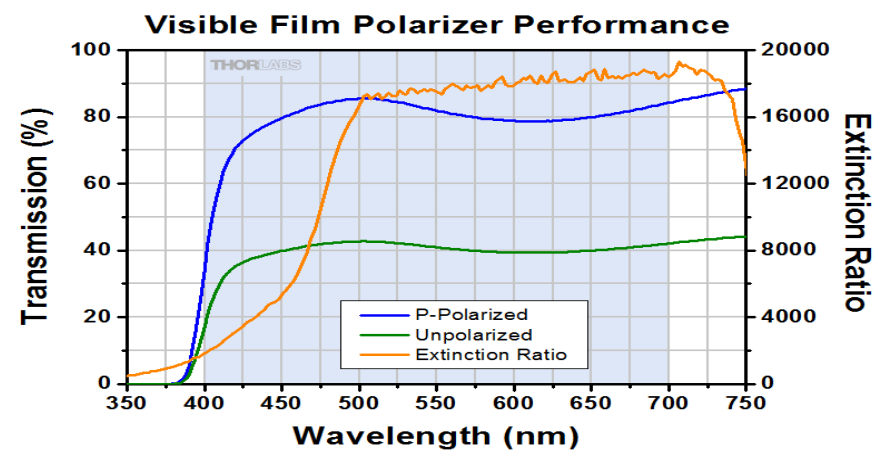

Figure 5.3: Transmission characteristics of polarizers at various wavelengths 
To obtain circularly polarised light from emitted linearly polarised light, quarterwave plates are used. Thorlabs quarter wave plates are fabricated from a liquid crystal polymer (LCP) sandwiched between two N-BK7 glass windows. The glass plates have a broadband anti-reflective (AR) coating for 350-700 $\mathrm{nm}$ range. LCP wave plates are designed to be true zero-order wave plates, which can provide stable performance over a range of wavelengths and within the range of angle of incidence (AOI) as shown in Figure 5.4. This design is useful for applications that require low sensitivity to AOI. A polymer wave plate can be used at an AOI up to $20 \%$ with $5 \%$ decrease in retardation, compared to less than $2^{0}$ for standard quartz wave plates. Due to the well-established spin coating process, polymer wave plates have excellent transmission uniformity across the wide aperture with low optical losses and low wavefront distortion of less than $\lambda / 4$ at $633 \mathrm{~nm}$.
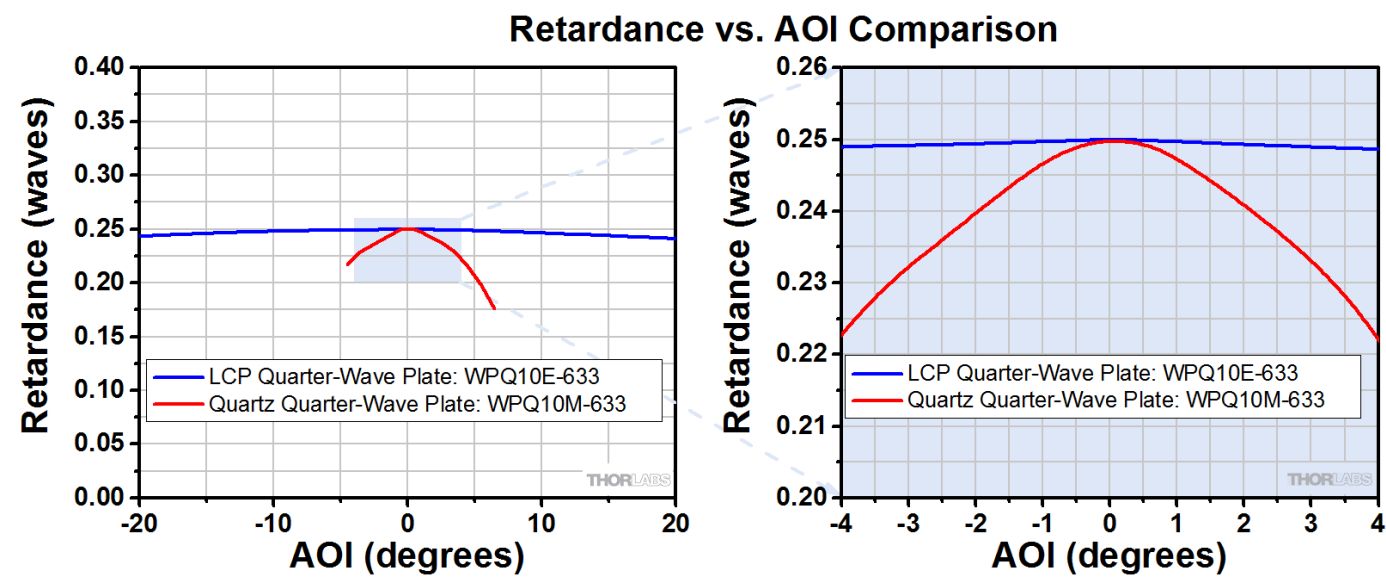

Figure 5.4: Retardance versus angle of incidence in degrees

Translational stage $(\mathrm{X}-\mathrm{Y})$ of aperture of $35 \mathrm{~mm}$ diameter and XY travel of 100 $\mathrm{mm}$ by $100 \mathrm{~mm}$ is designed for the specimen. The stage is lightweight and portable with a minimum resolution of $0.1 \mathrm{~mm}$. It has a rack and pinion gear system that can be driven both in $\mathrm{X}$ and $\mathrm{Y}$ axis with ball bearing slides that 
provides steady and smooth motion. It has a compact design and size that can be directly mounted to the microscope. The analyser is fitted on the top before the detector. The phase shifted images are obtained from the motorised analyser. The motorised precision rotation stage (PRM1/MZ8-THORLABS) is attached to the detector. It has $0.01^{0}$ resolution with high accuracy and precision. The software algorithm can be directly integrated into the system thus making the system completely automated. CCD detector DMK41AF02 of $1600 \times 1200$ pixels resolution from imaging source is deployed in the setup to capture the phase shifted images of the specimen.

\subsection{System Features}

The system comprises of following features

$>$ Residual stress and birefringence in 3D printed and injection moulded components

$>$ Static stress analysis with FEM validation

$>$ Defects and crack inspection of optical materials (e.g. Fused silica, crystals, lenses)

$>$ Qualitative and quantitative inspection of biomedical specimen

$>$ Dynamic stress analysis with wide FOV (Field of view)

$>$ Polymer material (PMMA, COC, epoxy) defect inspection

$>$ Modular packing

Normalised retardation in $\mathrm{nm} / \mathrm{cm}$ and integrated stress values 


\subsection{Maximum Likelihood estimation of the measurement system}

Reliability of measurement system is assessed by computing accuracy and precision of its measured values. Several tests are performed to check the distribution of measured values lies within standard normal distribution curve. Fifty sample tests were performed and their measurement deviation are computed. Likelihood function of the datasets is calculated and all of the values are bounded within two sigmas' $( \pm 2 \sigma)$ on both sides from their mean $(\mu)$ value. Thus, measured values are bounded within $95 \%$ confidence level of the normal standard distribution curve as shown in Figure 5.5.

For the normal distribution $N\left(\mu, \sigma^{2}\right)$, the probability density function is given by,

$$
f\left(x \mid \mu, \sigma^{2}\right)=\frac{1}{\sqrt{2 \pi \sigma^{2}}} \exp \left(-\frac{(x-\mu)^{2}}{2 \sigma^{2}}\right)
$$

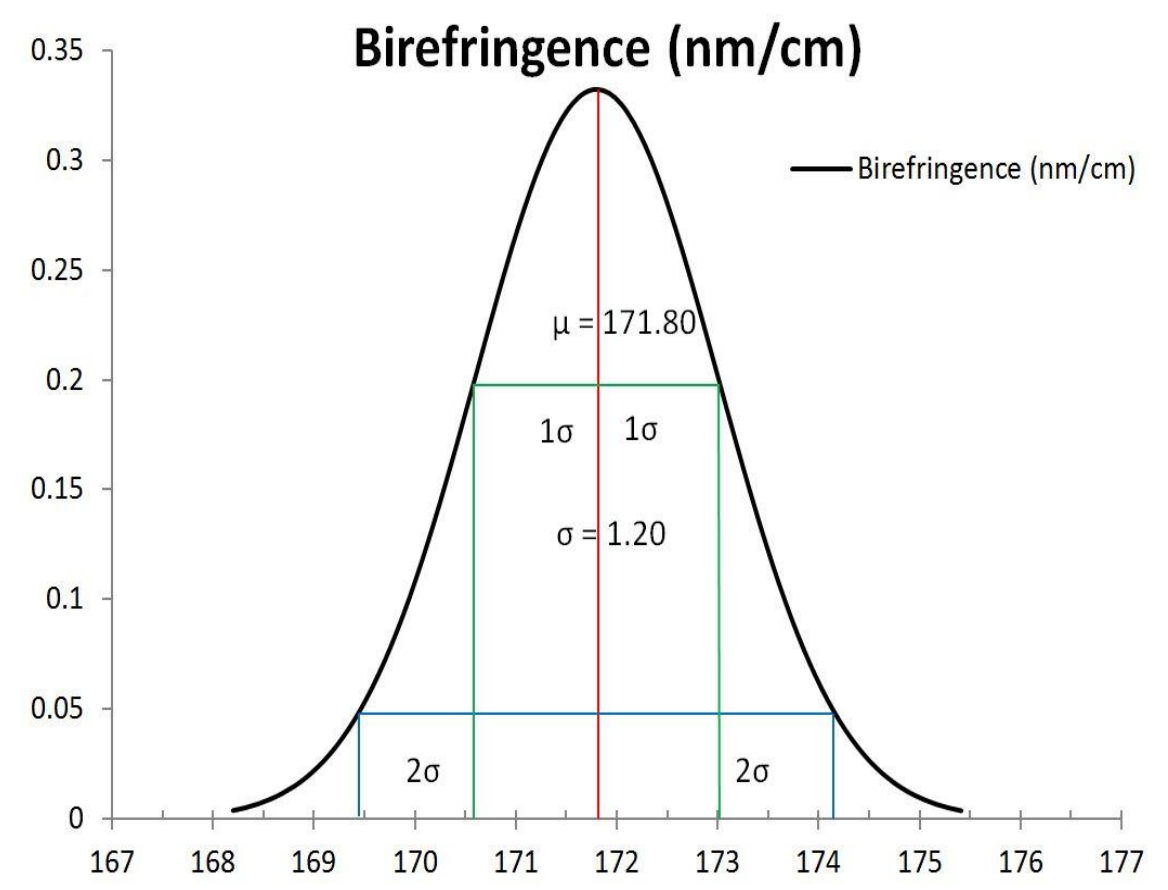

Figure 5.5: Maximum likelihood estimation (Standard distribution of the sample) 
Further analysis was carried out based on its scatter plot which represents the accuracy and precision of the measurement system as shown in Figure 5.6.

Scatter plot is plotted to observe its measured values and their mean value is calculated from fifty sample tests. The dispersed birefringence values is thoroughly observed in scatter plot with its normal distribution as represented in the previous figure. The samples were repetitively measured for assessing the precision and accuracy of the measurement system. The precision and accuracy of the measurement system is computed based on the available data and scatter plot was plotted within datasets as shown in Figure 5.6.

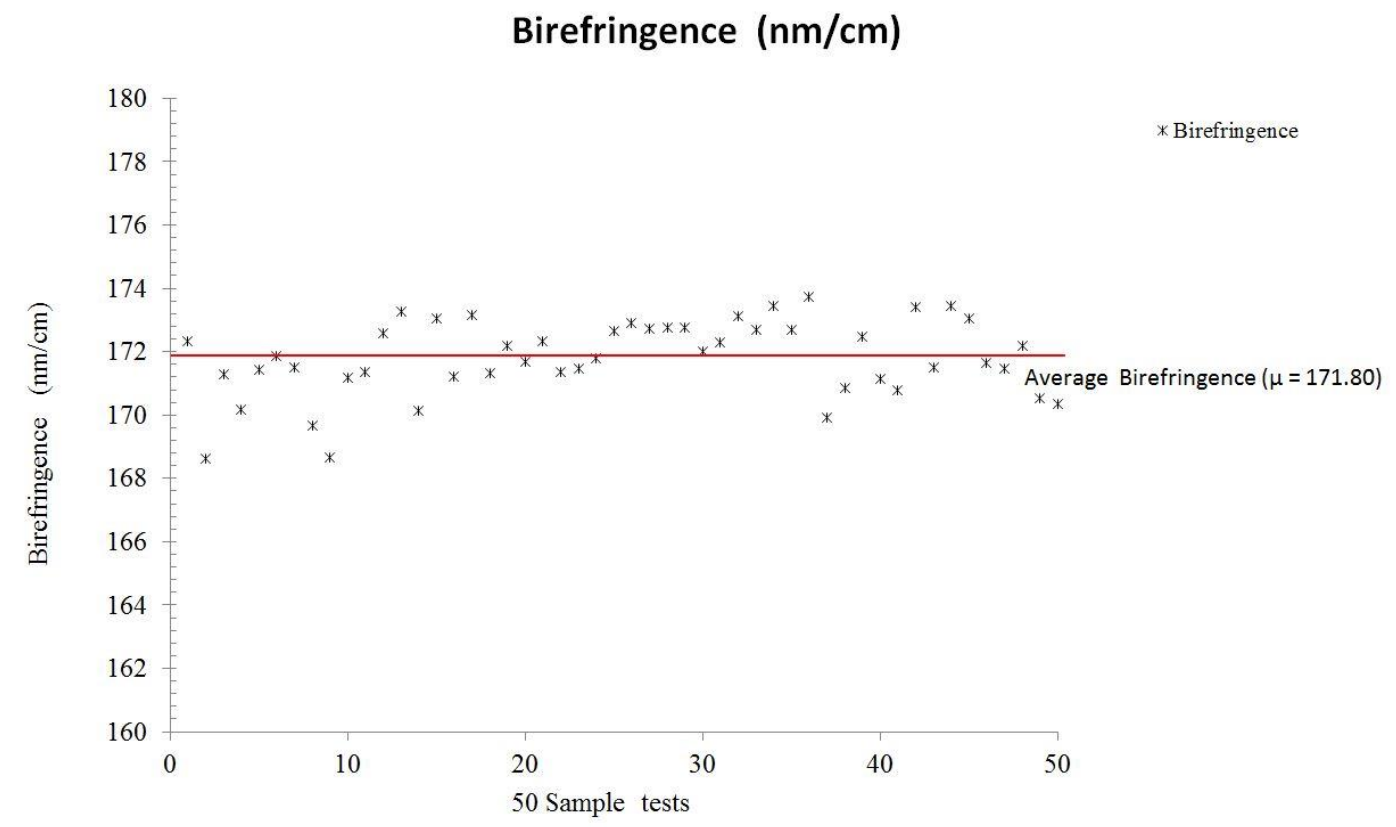

Figure 5.6: Scatter plot of the sample data normal distribution 


\subsection{Measurement Uncertainty}

Birefringence measurement of the system is well defined by the equation (5.13).

The resolution of the measurement system is calculated by computing minor deviation in birefringence value with respect to minimal increment in stress. The calculated resolution of the measurement system is $0.1 \mathrm{~nm} / \mathrm{cm}$. Further, we should compute the uncertainty of the measurement system. The uncertainty of the measurement system is entirely dependent on its factors during measurement. Firstly, the uncertainty of each component of the measurement system is analysed in detail. Afterwards, its value is integrated into the measurement system.

$$
B r=\frac{\Delta \times \lambda}{2 \pi \times t}
$$

The uncertainty of the birefringence measurement system is determined by the following equation,

$$
\Delta B r=\sqrt{\left(\frac{\partial B r}{\partial \Delta} \sigma \Delta\right)^{2}+\left(\frac{\partial B r}{\partial \lambda} \sigma \lambda\right)^{2}+\left(\frac{\partial B r}{\partial t} \sigma t\right)^{2}}
$$

where

$$
\begin{array}{llll}
\frac{\partial B r}{\partial \Delta}= & \frac{\partial}{\partial \Delta}\left(\frac{\Delta \times \lambda}{2 \pi \times t}\right) & =\frac{\lambda}{2 \pi \times t} \\
\frac{\partial B r}{\partial \lambda}= & \frac{\partial}{\partial \lambda}\left(\frac{\Delta \times \lambda}{2 \pi \times t}\right) & =\frac{\Delta}{2 \pi \times t} \\
\frac{\partial B r}{\partial t}= & \frac{\partial}{\partial t}\left(\frac{\Delta \times \lambda}{2 \pi \times t}\right) & =-\frac{\Delta \lambda}{2 \pi t^{2}}
\end{array}
$$

The measurement uncertainty is calculated for PMMA specimen of thickness 2.5 $\mathrm{mm}$ at $550 \mathrm{~nm}$ wavelength. The phase is calculated from phase shift measurement 
system and is found to be 0.49 . We calculated the deviation in phase, wavelength and thickness incurred in the birefringence measurement system.

Thus,

$\frac{\partial B r}{\partial \Delta}(\Delta, t, \lambda)=\frac{\partial}{\partial \Delta}\left(\frac{\Delta \times \lambda}{2 \pi \times t}\right)=\frac{\lambda}{2 \pi \times t}=\frac{550}{2 \pi \times 0.25}=350.14$

$\frac{\partial B r}{\partial \lambda}(\Delta, t, \lambda)=\frac{\partial}{\partial \lambda}\left(\frac{\Delta \times \lambda}{2 \pi \times t}\right)=\frac{\Delta}{2 \pi \times t}=\frac{0.49}{2 \pi \times 0.25}=0.3124$

$\frac{\partial B r}{\partial t}(\Delta, t, \lambda)=\frac{\partial}{\partial t}\left(\frac{\Delta \times \lambda}{2 \pi \times t}\right)=-\frac{\Delta \lambda}{2 \pi t^{2}}=\frac{0.49 \times 550}{2 \pi \times(0.25)^{2}}=-687.38$

As per the measurement rule, the deviation in measurement is always $20 \%$ of the resolution of measurement system.

Resolution of the Vernier calliper measurement system $=0.01 \mathrm{~mm}=0.001 \mathrm{~cm}$

Resolution of spectrometer for measuring wavelength of the source $=0.01 \mathrm{~nm}$

Phase resolution of the measurement system $=0.004$

Hence on calculating uncertainty of the measurement system we get,

$$
\begin{aligned}
U & =\sqrt{(350.14 \times 0.2 \times 0.004)^{2}+(0.3124 \times 0.2 \times 0.01)^{2}+(-687.38 \times 0.2 \times 0.001)^{2}} \\
& = \pm 0.0312 \mathrm{~nm} / \mathrm{cm}
\end{aligned}
$$

Thus, the uncertainty of the measurement system is equal to $\pm 0.0312 \mathrm{~nm} / \mathrm{cm}$. Position measurement with uncertainty is close to the Cramer-Rao lower bound estimation which is provided in the next section. As per the rule of thumb, uncertainty of the measurement system is 20 percent of the resolution of the measurement system. To verify the resolution of the measurement system as per the rule of thumb, 


$$
\begin{gathered}
20 \% \text { of resolution }(x)=\text { Uncertainity }(0.0312) \\
x(\text { resolution })=0.15 \mathrm{~nm} / \mathrm{cm}
\end{gathered}
$$

Thus, the resolution of the measurement system by rule of thumb $(0.15 \mathrm{~nm} / \mathrm{cm})$ almost agrees with calculated value $(0.1 \mathrm{~nm} / \mathrm{cm})$ as discussed in the beginning of this section. The minor discrepancies in the resolution is due to linear and rotational vibration errors in the measurement system along with temperature and pressure fluctuation in the laboratory.

\subsection{Cramer-Rao Lower Bound Estimation (CRLB)}

Measurement uncertainty is further assessed by Cramer Rao lower bound estimation. The uncertainty of the measured data is bounded by the reciprocal of the Fisher information which is calculated from the likelihood function of the measured values. Theoretical proof and mathematical calculation are provided in detail for the phase shifted interferometry herein. Consider a set of observed data samples $X=X_{1}, X_{2}, X_{3}, \ldots X_{n}$ and $\emptyset$ is to be estimated from the observed samples. The received phase image is affected by zero mean noise when it travels across the medium. We have image received at the detector given by,

$$
x(n)=\frac{a^{2}}{2}(1+\sin \Delta \sin 2(\alpha-\beta))+w[n], \quad n=0,1,2 \ldots, N-1
$$

Here a is amplitude, $\alpha$ is the phase shift, $\beta$ is fast axis direction, $\Delta$ is the phase retardation to be estimated and $w[n]$ is a constant noise throughout the measurement with mean $\mu=0$ and variance $=\sigma^{2}$. We use CRLB to find an efficient estimator to estimate the phase component. To derive CRLB, we should have PDF (Probability Density Function) to begin with. The underlying noise is 
constant with its mean $\mu=0$ and variance $=\sigma^{2}$, the pdf of the observed sample gets affected by this noise is computed by a multivariate Gaussian distribution function.

$$
\begin{aligned}
& p(x ; \emptyset)=\prod_{n=0}^{N-1} \frac{1}{\sqrt{2 \pi \sigma^{2}}} \exp \left[\frac{-\left(x-\mu_{x}\right)^{2}}{2 \sigma^{2}}\right] \\
& p(x ; \emptyset)=\prod_{n=0}^{N-1} \frac{1}{\sqrt{2 \pi \sigma^{2}}} \exp \left[-\frac{\left(x[n]-\mu_{x}\right)^{2}}{2 \sigma^{2}}\right] \\
& p(x ; \emptyset)=\frac{1}{\left(2 \pi \sigma^{2}\right)^{N / 2}} \exp \left[-\frac{1}{2 \sigma^{2}} \sum_{0}^{N-1}\left(x[n]-\mu_{x}\right)^{2}\right]
\end{aligned}
$$

The sample mean is given by,

$$
\begin{aligned}
\mu_{x} & =\frac{1}{N} \sum_{n=0}^{N-1} x[n] \\
& =\frac{1}{N} \sum_{n=0}^{N-1}\left[\frac{a^{2}}{2}(1+\sin \Delta \sin 2(\alpha-\beta)+w[n]]\right. \\
& =\frac{1}{N} \sum_{n=0}^{N-1}\left[\frac{a^{2}}{2}(1+\sin \Delta \sin 2(\alpha-\beta)]+\frac{1}{N} \sum_{n=0}^{N-1} w[n]\right. \\
& =\frac{1}{N} \sum_{n=0}^{N-1}\left[\frac{a^{2}}{2}(1+\sin \Delta \sin 2(\alpha-\beta)]\right.
\end{aligned}
$$

The probability density function of the sample data values is re-written as

$$
p(x ; \emptyset)=\frac{1}{\left(2 \pi \sigma^{2}\right)^{N / 2}} \exp \left[-\frac{1}{2 \sigma^{2}} \sum_{0}^{N-1}\left(x[n]-\frac{a^{2}}{2}(1+\sin \Delta \sin 2(\alpha-\beta))^{2}\right]\right.
$$

The observed samples $\mathrm{x}[\mathrm{n}]$ are represented by the equation 5.24 , we derive the likelihood function by computing logarithmic function of probability density function (PDF). That is, $p(\boldsymbol{x} ; \emptyset)$ is simply rewritten as $\mathrm{L}(\boldsymbol{x} ; \emptyset)$. The log likelihood function is given by, 
$\ln L(\boldsymbol{x} ; \varnothing)=-\frac{N}{2} \ln \left(2 \pi \sigma^{2}\right)+\left[-\frac{1}{2 \sigma^{2}} \sum_{0}^{N-1}\left(x[n]-\frac{a^{2}}{2}(1+\sin \Delta \sin 2(\alpha-\beta))^{2}\right]\right.$

Next, we take the partial derivative of log likelihood function with respect to $\alpha$

$$
\begin{aligned}
\frac{\partial \ln L(x ; \emptyset)}{\partial \alpha} & =\frac{\partial}{\partial \alpha}\left[\left(-\frac{N}{2} \ln \left(2 \pi \sigma^{2}\right)+\left(\frac{-1}{2 \sigma^{2}} \sum_{0}^{N-1}\left(x[n]-\frac{a^{2}}{2}(1+\sin \Delta \sin 2(\alpha-\beta))^{2}\right)\right]\right.\right. \\
& =-\frac{1}{\sigma^{2}} \sum_{n=0}^{N-1}\left(x(n)-\frac{a^{2}}{2}(1+\sin \Delta \sin 2(\alpha-\beta))\right)\left(-2 a^{2} \sin \Delta \cos 2(\alpha-\beta)\right) \\
& =\frac{2 a^{2}}{\sigma^{2}} \sum_{n=0}^{N-1}\left(x(n)-\frac{a^{2}}{2}(1+\sin \Delta \sin 2(\alpha-\beta))\right)(\sin \Delta \cos 2(\alpha-\beta)) \\
& =\frac{2 a^{2}}{\sigma^{2}} \sum_{n=0}^{N-1}\left(x(n) \sin \Delta \cos 2(\alpha-\beta)-\frac{a^{2}}{2}\left(\sin \Delta \cos 2(\alpha-\beta)+\frac{1}{2} \sin ^{2} \Delta \sin 4(\alpha-\beta)\right)\right)
\end{aligned}
$$

Taking the second partial derivative of the log likelihood function,

$$
\begin{aligned}
& \frac{\partial^{2} \ln L(x ; \emptyset)}{\partial^{2} \alpha}=\frac{2 a^{2}}{\sigma^{2}} \sum_{n=0}^{N-1}\left(\left(x(n) \sin \Delta(-2 \sin 2(\alpha-\beta))-\frac{a^{2}}{2}\left(\sin \Delta(-2 \sin 2(\alpha-\beta))+\frac{1}{2} \sin ^{2} \Delta 4 \cos 4(\alpha-\beta)\right)\right.\right. \\
= & \frac{2 a^{2}}{\sigma^{2}} \sum_{n=0}^{N-1}\left(\left(\frac { a ^ { 2 } } { 2 } \left(1+\sin \Delta \sin 2(\alpha-\beta) \sin \Delta(-2 \sin 2(\alpha-\beta))-\frac{a^{2}}{2}\left(\sin \Delta(-2 \sin 2(\alpha-\beta))+\frac{1}{2} \sin ^{2} \Delta 4 \cos 4(\alpha-\beta)\right)\right.\right.\right. \\
= & -\frac{a^{4}}{\sigma^{2}}\left(1+\cos 4(\alpha-\beta)-\cos ^{2} \Delta(1+\cos 4(\alpha-\beta))\right.
\end{aligned}
$$

Since the above equation is still dependent on the observed samples $x[n]$, taking expectation of the entire equation to average out the variations.

$$
\begin{gathered}
-E\left[\frac{\partial^{2} \ln L(\boldsymbol{x} ; \emptyset)}{\partial^{2} \alpha}\right]=\frac{a^{4}}{\sigma^{2}}\left[\left(\sum_{n=0}^{N-1} 1+\sum_{n=0}^{N-1}\left(\cos 4(\alpha-\beta)-\cos ^{2} \Delta(1+\cos 4(\alpha-\beta))\right)\right]\right. \\
\approx \frac{a^{4}}{\sigma^{2}}[N-0]=\frac{N A}{\sigma^{2}}
\end{gathered}
$$

Amplitude term is represented by $\mathrm{A}=a^{4}$, thus Fisher information can be derived by using, 


$$
I(\varnothing)=-E\left[\frac{\partial^{2} \ln L(x ; \varnothing)}{\partial^{2} \alpha}\right]=\frac{N A}{\sigma^{2}}
$$

Cramer-Rao lower bound is the reciprocal of Fisher information.

$$
C R L B=\frac{1}{I(\varnothing)}=\frac{\sigma^{2}}{N A}
$$

The variance of any estimator estimating the phase information is always higher than this CRLB. That is,

$$
\operatorname{var}(\widehat{\varnothing}) \geq \frac{\sigma^{2}}{N A}
$$

As we can see from the above result, that the variance of the estimator, $\operatorname{var}(\widehat{\varnothing}) \rightarrow$ $C R L B$ as $N \rightarrow \infty$, which is represented by the Figure 5.7 and has been proved experimentally as well.

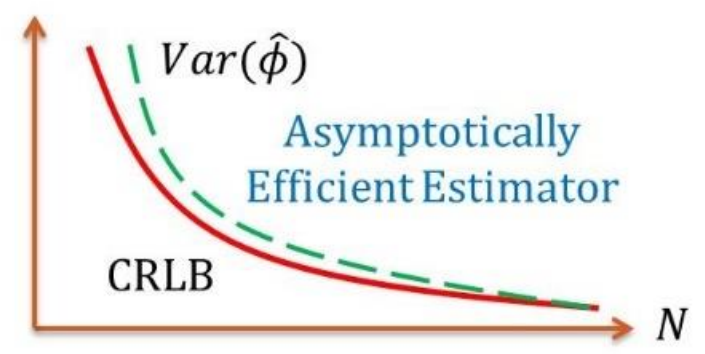

Figure 5.7: Relationship between CRLB and unbiased estimator

It is evident from the derivation that CRLB is the minimum variance of the unbiased estimator of the maximum likelihood estimation which is represented schematically in Figure 5.7 of the sample data values.

Experimental estimation of the measured values of fifty datasets is given by, $\sigma=1.202$ (as calculated from the fifty datasets) 
$\mathrm{N}=50$ (no of sample tests)

$\mathrm{A}=$ normalized amplitude $=1$ (same for all datasets)

$$
C R L B=\frac{\sigma^{2}}{N A}=\frac{(1.202)^{2}}{50}=0.0289
$$

On comparing CRLB value with the uncertainty ( $U=0.0312 \mathrm{~nm} / \mathrm{cm}$ ) of the measurement system, we found uncertainty of the measurement system is almost same to the calculated CRLB value. Thus, the uncertainty of the measurement system $(\mathrm{U}=0.0312 \mathrm{~nm} / \mathrm{cm})$ is bounded by Cramer-Rao lower bound value.

\subsection{New RGB plane polariscope algorithm}

In this setup, two plane polarizers are crossly arranged to obtain dark field plane polariscope. The four phase-shifted intensity images are computed to acquire the phase retardation magnitude and direction values. It is different from the existing system because it eliminates quarter wave plate by white light source and colour camera. A white light source and the colour camera has been used for recording four phase shifted images. In this setup, both polarizers and analysers are phase shifted in the interval of $22.5^{0}$ to retain dark field images. The four images are processed to obtain phase retardation magnitude and its direction. At four different phase shifted images $\left(0,22.5^{0}, 45^{0}, 67.5^{0}\right)$ the images recorded are $\mathrm{I}_{1}, \mathrm{I}_{2}$, $\mathrm{I}_{3}, \mathrm{I}_{4}$ respectively. The phase retardation $(\delta)$ and its direction $(\theta)$ are computed as follows:

$$
\begin{aligned}
& \text { Phase retardation }(\delta)=\sqrt{\left(I_{3}-I_{1}\right)^{2}+\left(I_{4}-I_{2}\right)^{2}} \\
& \text { Phase direction }(\theta)=\frac{1}{4} \tan ^{-1}\left(\frac{i_{4}-i_{2}}{i_{3}-i_{1}}\right)
\end{aligned}
$$


This dark field polariscope algorithm eliminates the requirement of expensive and bulky quarter wave plate. Implementation of this algorithm makes the system less complicated and cheap compared to the existing system. In addition to that, we use ordinary white light and colour camera; the experimental setup can be designed at any ordinary lab at a moderate cost which is the significant feature of this algorithm. Thus, this setup abrogates the requirement of the quarter wave plate and monochromatic light source simplifying the setup which is quite feasible in scientific labs or industries in terms of cost and space required. Moreover, we have validated white light source with fewer optical components and the system has a huge potential.

\subsection{Comparison of new algorithm with existing one}

New algorithm requires fewer components and has improved computational speed compared to existing algorithm. Various optical samples are measured and their results were compared for further analysis. Here, we compare results of the polymethylmethacrylate (PMMA) phase retardation (birefringence) values and its direction from the existing algorithm and the new one. The phase retardation and direction values are in good agreement with the existing system. Further research has been carried out to improve the results and the computational speed that will enhance the new algorithm crucial for rapid qualitative and quantitative inspection of the samples. 


\subsubsection{Existing algorithm results in low birefringence polariscope}

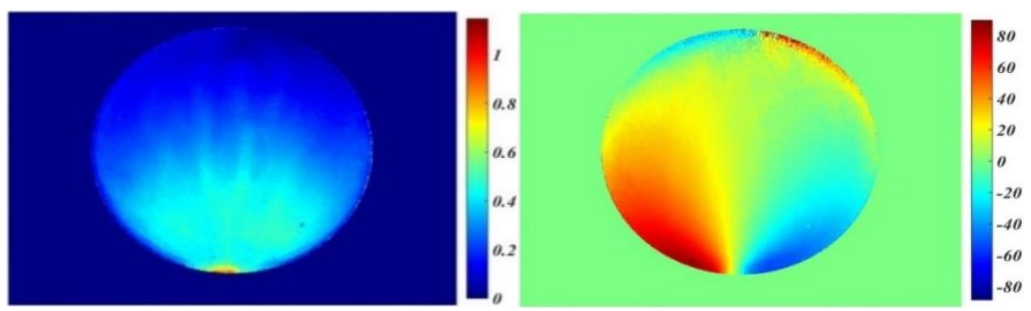

Figure 5.8: Phase retardation and direction of the PMMA sample by existing algorithm

The phase retardation and direction values in Figure 5.8 are from the previous low birefringence polariscope. We tried to compare the results provided by the existing algorithm with the new one as in Figure 5.9. Their comparative results seem to be in good agreement.

\subsubsection{RGB algorithm results for plane polariscope in dark field arrangement}
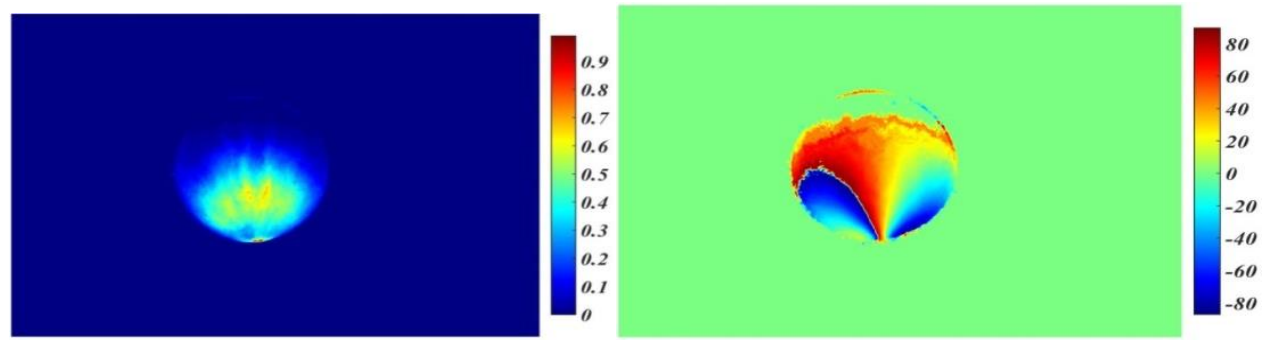

Figure 5.9: Phase retardation and direction of the PMMA sample by new algorithm

The phase retardation magnitude and its direction values are in well agreement with the existing system. This algorithm utilises few optical parts and has a better scope for further research and development. The phase image is distorted from the new algorithm due to the lack of exposure on the sample due to black field 
imaging. There is an ongoing improvement in the algorithm for enhanced phase image information.

\subsection{Product Testing and Evaluation}

The measurement was carried out for different injection moulded materials, for instance, PMMA disc, microfluidic channels and others to verify the reliability of the system. The measurement is accurate, convincing and is on par with the existing system. The automatic whole field retardation, birefringence and stress analysis is the salient feature of this system as shown in Figure $\mathbf{5 . 1 0}$ and Figure 5.11. The system is entirely tuneable for wavelength dependent material and can be measured from smaller to the bigger size of specimen eliminating the necessity of sample stitching algorithm. The manufactured system is a testament for research institutes particularly SIMtech and FluidGM for qualitative and quantitative inspection of the plastic samples.

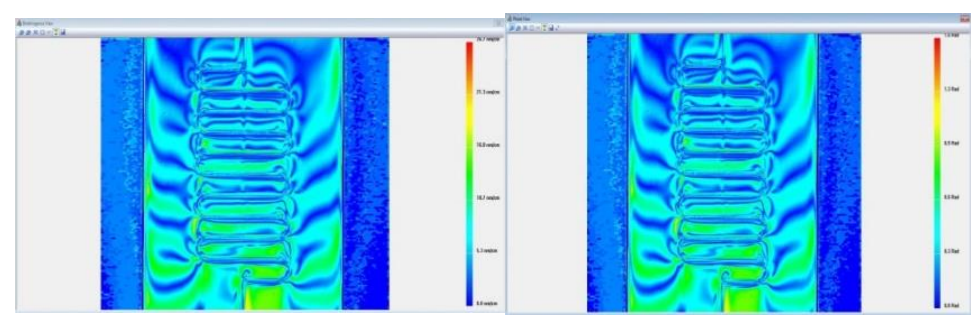

Figure 5.10: Birefringence and phase view of the microfluidic channels

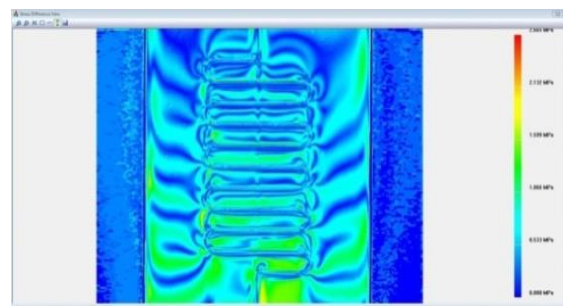

Figure 5.11: Integrated Residual stress in Microfluidic Channels 


\subsection{Software Interface}

The polariscope software snapshot is shown in Figure $\mathbf{5 . 1 2}$ with cascaded windows providing detailed guided user interface (GUI). It provides phase difference along with direction, birefringence values, stress difference along with direction and plot map features within sample image. The wavelength of the light source, photoelastic constant and thickness of the sample is loaded in the software for fulfilling required sample properties. The software is written in $\mathrm{C}++$ platform based on Microsoft Foundation class library and has a robust speed which is incumbent feature in software. The sample is PMMA with two-point loading along the horizontal axis where we can observe the stress difference and mapped birefringence values. Moreover, we can plot phase retardation along the various diameter of the sample to observe the deflection or deformation pattern in the sample. The software is real time imaging hence we can see the defect or crack propagation in the sample under any perturbation or application of load.

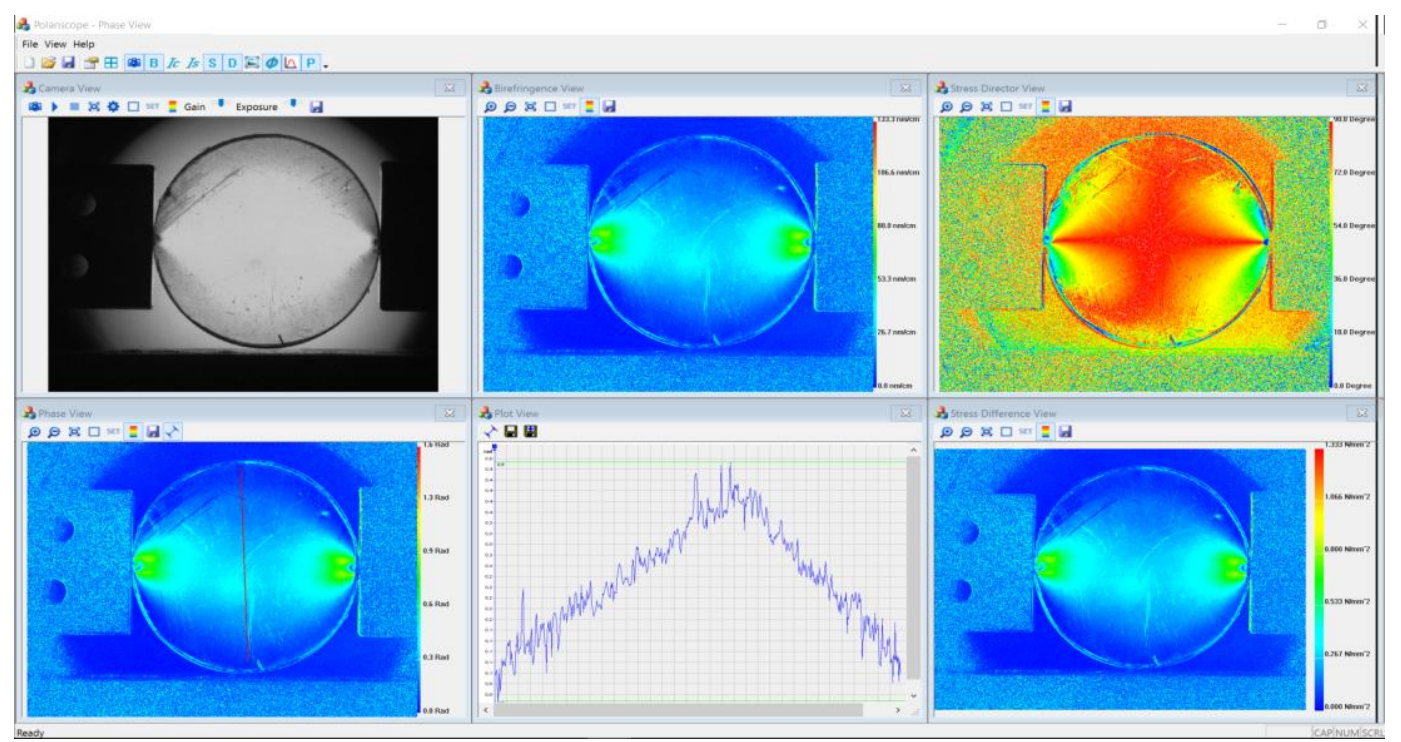

Figure 5.12: Software interface of low birefringence measurement system 


\subsection{Summary}

In this chapter, we have evolved and shifted our research platform setup to a commercial setup essential in industry, research labs and commercial companies. The qualitative and quantitative inspection of the optical specimen is imperative for identifying forthcoming defects and cracks in the material. Minor defects in the optical sample causes intrinsic error in the measurement crucial for microwells and microfluidic channels during sample inspection. Micro wells and micro fluidic channels is used for biological mimicking application hence requires delicate and thorough measurement. Thus, the design of automated birefringence measurement system is essential for diagnosing micro wells and microfluidic channels in biological domain.

The system is completely automatic applicable for rapid testing of the samples instantaneously without any issues. It is automatic whole field high-resolution measurement system with wide field of view (FOV) useful for real-time application compared to the existing commercial system. The prevalent commercial system has its own drawbacks since it's a point to point measurement system with limited resolution, time-consuming and can't map out the true shape or curve of the specimen. Moreover, it can't measure wavelength dependent optical samples (optical windows) particularly red filter which is mentioned in the previous chapter because of its monochromatic light source nature. This system can be deployed for inspecting defects and cracks in optical filters ranging from infra-red to visible wavelength. It has been deployed for inspecting defects in Si(Silicon) wafers. Hence, we demonstrate that the LBP system can be a reliable tool for providing a rapid non-destructive measurement of residual stress in 
injection moulded materials. This is vital in quality control of low-cost plastic, $\mathrm{Si}$ wafers and glass components especially in the growing areas of 3D printed plastic lens. 


\section{Chapter 6: Conclusions and Recommendations for Future Work}

This chapter provides the summary of the entire work carried out for birefringence effects in plastic optics. The future work based on the research conducted in birefringence of injection moulded optics has been recommended.

\subsection{Conclusions}

In the literature review, we are acquainted with prevalent birefringence measurement systems. From the literature review, we discovered that there is an immense potential of single shot whole field birefringence measurement system for qualitative and quantitative inspection of the plastic optics samples. The nonuniformities of the plastic material is the critical issue for manufacturing companies, imaging system and for R\&D (Research and development). Thus, in this research, we have conducted the measurement of every injection moulded plastics optics and the best way to measure it.

$>$ The birefringence testing was performed in various plastic optics particularly lenses, channels, composites, diamond, glass, microplates and syringe. The birefringence is induced because of material non-uniformities due to residual stress, temperature gradient and many other factors. Calcite, the birefringent sample is modelled in ZEMAX, where two images are formed along the ordinary and extraordinary axis because of its birefringent properties. The mathematical modelling of birefringence is based on Jones calculus and image intensity is formulated by Fourier 
transformation method. Image degradation due to birefringence is computed and analysed by a statistical approach, which is square proportional to the contrast and intensity of the material. Stress induced birefringence is computed by finite element modelling and compared with the theoretical and mathematical methods and the results are in well agreement.

Microfluidic channels are widely used specimen in biological labs for cancer research and mimicking biological phenomenon. Qualitative and quantitative inspection of the manufactured microfluidic channel is vital since it will be deployed for biological applications. Quantitative inspection of biological optical samples is assessed via birefringence measurement system concerned in anisotropies or non-uniformities of the channels. The anisotropies in the channel distorts measurement values. Highly anisotropic samples are undesirable and are designated as faulty samples during product selection and verification. Thus, for proper selection of the microfluidic channels, thorough birefringence measurement of the sample must be carried out. So, a circular sample of the same material produced in the same batch is calibrated through a standard calibration procedure as a part of the measurement. The procedure is rapidly and repeatedly investigated at different applied force to confirm the reproducibility of photoelastic constant value of the samples. Photoelastic constant computed by calibration procedure is mapped into phase difference values of microfluidic channels to obtain birefringence values. These measured birefringence readings are crucial 
for qualitative and quantitative assessment required for product selection and verification.

Optical windows (filters) are wavelength dependent material that only responds on a certain wavelength. This unique feature makes it suitable for optical sensors. The provided optical windows are red filters hence responsive for red colour wavelength. Since the quarter wave plates were designed for green colour wavelength, the compensation matrix was computed for the red filters and is implemented to obtain the true birefringence values. The measured birefringence values comply with industry standard values justifying the applied approach. Optical windows (red filters) are used in conveyor belt sensors hence crucial and requires a thorough inspection.

After all the research and measurement conducted, we came with an idea to design an automated system that will be convenient in the industry, research and development for sample selection and verification. The LED (Light emitting diode) arrays in-built microprocessor (Arduino) is used in which wavelength and intensity are tuneable. It is applicable for wavelength and intensity dependent material. To obtain uniformly diffused and collimated beam, the holographic diffuser is placed on top of LED array source. The stage is translational and robust uninfluenced by vibration with a wide field of view (FOV) suitable for wide specimens (75 $\mathrm{mm} \times 75 \mathrm{~mm}$ ). Above all, the analyser of the measurement system is automatic and manipulated by software, hence captures the automatic phase-shifted images to obtain the birefringence values. 
$>$ The birefringence measurement system was thoroughly investigated with an aim to optimise the results, time and cost. So, mathematical relations and algorithms have been completely optimised with an objective to minimize the computation time and components required. The mathematical relation and algorithm are designed for white LED source, colour camera and two linear polarisers. The birefringence results obtained from new algorithm and arrangement is in good agreement with the existing system. Noble optimised system with fewer components has efficient algorithm thus reduces computation time convenient for scientific laboratories and industries. It can be made portable and integrated into the existing imaging system.

\subsection{Recommendations for Future Work}

The work conducted in this thesis has a tremendous scope in the qualitative and quantitative inspection of plastic optics. The probable future directions of the research work reported in this thesis are as follows:

The birefringence measurement of plastic optics has been conducted for the samples that were already manufactured. In-situ birefringence measurement is essential during mass manufacturing because it economise both time and cost. On integrating birefringence measurement system in plastic optics manufacturing industries, it will provide onsite sample selection along with continuous monitoring. Furthermore, portable birefringence measurement system will be very useful in biological labs 
for qualitative and quantitative inspection of optical samples. The current existing system can be made portable and inexpensive since it has improved algorithm with fewer components.

The new algorithm is implemented in visible light photoelasticity and gave a similar result to the existing algorithm. This algorithm requires fewer components and has high computational speed. This algorithm has been implemented in IR (Infra-red) range and its results are found to be almost same. Once the algorithm is successfully implemented in IR range, IR system will be inexpensive compared to the existing IR system. IR system will have a bright scope in silicon wafers industry for defects and crack analysis.

Subwavelength characterisation of the periodic samples can be carried out by Mueller matrix analysis in birefringence measurement system. The polarimeter is manually rotated at small angles to get the periodic information of the samples. The experimental research has been conducted and results were published in the journal. Since this system is now fully automatic, the subwavelength metrological characterisation is straightforward and has good prospects for periodic subwavelength grating structure.

When the system is in bright field mode, visible scratches can be spotted whereas in cross-field mode we can detect digs along with scratches. The system can be used to inspect scratch and dig along with birefringence. 
Currently, the system has been used for birefringence measurement and has a bright prospect for scratch and dig analysis.

Birefringence measurement system captures the polarised image of the sample at different polarisation angles and orientations. For specular surfaces at different polarisation angles, it has different shaded images. It captures shaded images at different polarisation angles which will be helpful to reconstruct three-dimensional (3D) profile of the specular surfaces. Thus, this system will be applicable in 3D profiling of specular surfaces. Non-invasive 3D profiling of specular specimen is still a challenging task in metrology. 


\section{LIST OF PUBLICATIONS}

Papers published:

1. Adhikari, A., \& Asundi, A. (2015, March). Birefringence characterization of injection moulded microplates. In International Conference on Experimental Mechanics 2014 (pp. 93022Q-93022Q). International Society for Optics and Photonics.

2. Adhikari, A., \& Asundi, A. (2015, September). Modelling, simulation, and analysis of birefringent effects in plastic optics. In SPIE Optical Systems Design (pp. 96260I-96260I). International Society for Optics and Photonics

3. Adhikari, A., Dev, K., \& Asundi, A. (2016). Subwavelength metrological characterization by Mueller matrix polarimeter and finite difference time domain method. Optics and Lasers in Engineering, 86, 242-247.

4. Adhikari, A., \& Asundi, A. (2016). Residual stress measurement for injection moulded components. Theoretical and Applied Mechanics Letters, 53, 122-128.

5. Adhikari, A., Dev, K., \& Asundi, A. (2016). Subwavelength metrology of Al wire grating employing finite difference time domain method and Mueller matrix polarimeter. In SPIE Optical and Photonics (pp. 99960I9700I). International Society for Optics and Photonics 
6. Adhikari, A., Cheng, H., Khoo, R., \& Asundi, A. (2016).Birefringence measurement of optical windows. In Asian Conference on Experimental Mechanics (pp. 18235-18333).

7. Guan, L., Ding, J., Du, L., Adhikari, A., \& Asundi, A. K. (2017, June). Spectroscopic imaging ellipsometry for thin film detection on uniaxial crystal. In Fifth International Conference on Optical and Photonics Engineering (pp. 1044921-1044921). International Society for Optics and Photonics. 


\section{REFERENCES}

1. Beich, W.S. Injection molded polymer optics in the 21st century. in Optics \& Photonics 2005. 2005. International Society for Optics and Photonics.

2. Bäumer, S., Applications of Injection-Molded Optics. John Wiley \& Sons, 2010: p. 251-286.

3. Claude Tribastone, W.P., An Introduction to the Design, Manufacture and Application of Plastic Optics. 1995.

4. Lee, Y., et al., Investigation on residual stress and stress-optical coefficient for flexible electronics by photoelasticity. Measurement, 2012. 45(3): p. 311-316.

5. Malacara, D., Optical shop testing. Vol. 59. 2007: John Wiley \& Sons.

6. Smartt, R. and W. Steel, Birefringence of quartz and calcite. J. Opt. Soc. Am, 1959. 49: p. 710-712.

7. Kaminsky, W., K. Claborn, and B. Kahr, Polarimetric imaging of crystals. Chem Soc Rev, 2004. 33(8): p. 514-25.

8. Bäumer, S., Metrology of Injection Molded Optics. John Wiley \& Sons, 2010: p. 67-122.

9. Phillips, J.W., Photoelasticity. University of Illinois at UrbanaChampaign, 1998: p. 6-2-6-62.

10. Crawford, S. and H. Kolsky, Stress birefringence in polyethylene. Proceedings of the Physical Society. Section B, 1951. 64(2): p. 119.

11. Sun, C.-1. and H.-Y. Huang, Measurements of flow-induced birefringence in microfluidics. Biomicrofluidics, 2016. 10(1): p. 011903.

12. Wu, S.-T., U. Efron, and L.D. Hess, Birefringence measurements of liquid crystals. Applied optics, 1984. 23(21): p. 3911-3915.

13. Unno, Y., Distorted wave front produced by a high-resolution projection optical system having rotationally symmetric birefringence. Applied optics, 1998. 37(31): p. 7241-7247.

14. Unno, Y., Influence of birefringence on the image formation of high resolution projection optics, in Optical Society of America. 2000. p. 3243-3252.

15. Unno, Y., Analyses of imaging performance degradation caused by birefringence residual in lens materials. Journal of Micro/Nanolithography, MEMS, and MOEMS, 2002. 1(1): p. 49.

16. Gerber, R.E. and M. Mansuripur, Effects of substrate birefringence and tilt on the irradiance and phase patterns of the return beam in 
magneto-optical disk data storage. Applied Optics, 1995. 34(22): p. 4780-4787.

17. Tribastone, C., C. Gardner, and W.G. Peck. Precision plastic optics applications from design to assembly. 1995.

18. Minami, K., Optical Plastics. 2010: John Wiley \& Sons.

19. Thomas Bauer, D., Tooling for Injection Molded Optics. 2010: WILEY-VCH Verlag GmbH \& Co. KGaA, Weinheim.

20. Fang, M., et al., Polarization-sensitive optical projection tomography for muscle fiber imaging. Scientific reports, 2016. 6.

21. Sachs, E.M., et al., Three-dimensional printing techniques. 1993, Google Patents.

22. Willis, K., et al. Printed optics: $3 D$ printing of embedded optical elements for interactive devices. in Proceedings of the 25th annual ACM symposium on User interface software and technology. 2012. ACM.

23. Zhang, C., et al., Open-source 3D-printable optics equipment. PloS one, 2013. 8(3): p. e59840.

24. Bäumer, S., Handbook of Plastic Optics. 2011: Wiley.

25. Davis, A., Microstructured Optics for LED Applications. Reflexite Display Optics, 2002.

26. Kuntze, T., Plastic Optics Enable LED Lighting Revolution. Optik \& Photonik, 2007. 2(4): p. 42-45.

27. Deutsch, T.F., Lasers and optics in health care. Proceedings of the IEEE, 1997. 85(11): p. 1797-1816.

28. Bilro, L., et al., Optical sensors based on plastic fibers. Sensors, 2012. 12(9): p. 12184-12207.

29. Mäkinen, J.-T. and R. de Schipper, How can plastics injection molding help driving down the cost of photovoltaic concentrators?

30. Miñano, J.C., et al., Free-form optics for Fresnel-lens-based photovoltaic concentrators. Optics express, 2013. 21(103): p. A494A502.

31. Buljan, M., et al. Improving performances of Fresnel CPV system: Fresnel-RXI Köhler concentrator. 2010. European Photovoltaic Solar Energy Conference and Exhibition (EU PVSEC).

32. Xie, W., et al., Concentrated solar energy applications using Fresnel lenses: A review. Renewable and Sustainable Energy Reviews, 2011. 15(6): p. 2588-2606. 
33. Wu, Y., et al., Experimental characterisation of a Fresnel lens photovoltaic concentrating system. Solar Energy, 2012. 86(1): p. 430-440.

34. Shvarts, M., et al. Flat-plate Fresnel lenses with improved concentrating capabilities: designing, manufacturing and testing. in Photovoltaic Specialists Conference, 2008. PVSC'08. 33rd IEEE. 2008. IEEE.

35. Antón, J.C.M., et al. Method for the characterization of Fresnel lens flux transfer performance. in SPIE Optical Systems Design. 2011. International Society for Optics and Photonics.

36. Vázquez-Moliní, D., et al. New concentrator multifocal Fresnel lens for improved uniformity: design and characterization. in SPIE Solar Energy+ Technology. 2009. International Society for Optics and Photonics.

37. Davis, A. and F. Kühnlenz, Optical design using Fresnel lenses. Optik \& Photonik, 2007. 2(4): p. 52-55.

38. Menendez, J., F. Erismann, and M. Gauvin, The advantages of plastic optical components. Optics and Photonics News, 1999. 10(8): p. 28-30.

39. Katte, H., Imaging measurement of stress birefringence in optical materials and components Photonik International 2009: p. 39-41.

40. Koechner, W. and D. Rice, Effect of birefringence on the performance of linearly polarized YAG: Nd lasers. IEEE Journal of Quantum Electronics, 1970. 6(9): p. 557-566.

41. https://en.wikipedia.org/wiki/Birefringence.

42. Saito, T.I.a.H., Funct. Mater. 3, 1987. 25.

43. Pantani, R., Validation of a model to predict birefringence in injection molding. European Polymer Journal, 2005. 41(7): p. 14841492.

44. Kim, S.Y., et al., Measurement of residual stresses in film insert molded parts with complex geometry. Polymer Testing, 2009. 28(5): p. 500-507.

45. Brewster, D., On the communication of the structure of doublyrefracting crystals to glass, murite of soda, flour spar, and other substances by mechanical compression and dilation. Phil Trans $\mathrm{R}$ Soc London 1816: p. 156-78.

46. Wang, D. and R. Liang, Simultaneous polarization Mirau interferometer based on pixelated polarization camera. Optics letters, 2016. 41(1): p. 41-44. 
47. Serrano-Garcia, D.I. and Y. Otani, Dynamic phase measurements based on a polarization Michelson interferometer employing a pixelated polarization camera. Advanced Optical Technologies, 2017. 6(1): p. 47-51.

48. Quiroga, J. and A. González-Cano. Phase measurement method for stress analysis from photoelastic data. in IUTAM Symposium on Advanced Optical Methods and Applications in Solid Mechanics. 2000. Springer.

49. Ghosh, N. and K. Bhattacharya, Polarization phase-shifting interferometric technique for complete evaluation of birefringence. Applied optics, 2011. 50(15): p. 2179-2184.

50. Gdoutos, T., Matrix theory of photoelasticity. 1979, Springer Series in Optics.

51. Wong, C., Birefringence measurement using a photoelastic modulator. Applied Optics, 1979. 18(23): p. 3996-3999.

52. Chipman, R.A., Polarization analysis of optical systems. Optical Engineering, 1898. 28(February 1989): p. 090 -099.

53. Sanyal, S., et al., Frequency response characteristics of a birefringent lens with off-axis aberrations. Applied optics, 2004. 43(19): p. 3838-3847.

54. Lesso, J.P., et al., Aberrations introduced by a lens made from a birefringent material. Applied optics, 2000. 39(4): p. 592-598.

55. Katte, H., Measuring of High Quality Optics for Lithography. Optik \& Photonik, 2009. 4(2): p. 26-29.

56. Xia, Y. and G.M. Whitesides, Soft lithography. Annual review of materials science, 1998. 28(1): p. 153-184.

57. Takahashi, A., et al., Influence of birefringence on the signal quality of magnetooptic disks using polycarbonate substrates. Applied Optics, 1988. 27(14): p. 2863-2866.

58. Unno, Y., Point-spread function for a rotationally symmetric birefringent lens. JOSA A, 2002. 19(4): p. 781-791.

59. Katte, H., Imaging measurement of stress birefringence in optical materials and components. Photonik international, 2009. 1(2009): p. 39-41.

60. Russell A. Chipman, L.J.C., Polarisation aberration diagrams derivations. Optical Engineering 1989. 28(2)(February 1989): p. 100-106

61. Chipman, R.A., Polarization analysis of optical systems. Optical engineering, 1989. 28(2): p. 280290-280290. 
62. Keith B. Doyle, J.M.H., Victor L. Genberg \& Gregory J. Michels, Stress Birefringence Modeling for Lens Design and Photonics.pdf. 2003.

63. Unno, Y., Influence of birefringence on the image formation of highresolution projection optics. Applied Optics, 2000. 39(19): p. 32433252.

64. Roy-Br-honnet, F.L., et al., Optical media and target characterization by Mueller matrix decomposition. Journal of Physics D: Applied Physics, 1996. 29(1): p. 34.

65. Jones, R.C., A New Calculus for the Treatment of Optical Systems. Journal of the Optical Society of America, 1941. 31(7): p. 488-493.

66. Gnatowski, A., P. Palutkiewicz, and K. Lubczyñska, Analysis of stress state in DMTA and photoelasticity examinations. Archives of Materials Science, 2010. 40: p. 40.

67. Dora, P.T. and K. Ramesh. Measurement of residual birefringence in thin glass plates using digital photoelasticity. in International Conference on Experimental Mechanics 2013 and the Twelfth Asian Conference on Experimental Mechanics. 2014. International Society for Optics and Photonics.

68. Gwag, J.S., et al., Novel cell gap measurement method for a liquid crystal cell. Japanese journal of applied physics, 2002. 41(1A): p. L79.

69. Kim, C.H. and J.R. Youn, Determination of residual stresses in injection-moulded flat plate: Simulation and experiments. Polymer Testing, 2007. 26(7): p. 862-868.

70. Doyle, K.B., et al. Stress birefringence modeling for lens design and photonics. in International Optical Design Conference 2002. 2002. International Society for Optics and Photonics.

71. Yonezawa, K., Y. Kozawa, and S. Sato, Generation of a radially polarized laser beam by use of the birefringence of a c-cut $\mathrm{Nd}$ : YVO4 crystal. Optics letters, 2006. 31(14): p. 2151-2153.

72. Czarske, J.W. and H. Mueller, Birefringent Nd: YAG microchip laser used in heterodyne vibrometry. Optics communications, 1995. 114(3-4): p. 223-229.

73. Ren, C. and S. Zhang, Diode-pumped dual-frequency microchip Nd: YAG laser with tunable frequency difference. Journal of Physics D: Applied Physics, 2009. 42(15): p. 155107.

74. Liu, W., et al., Differential variation of laser longitudinal mode spacing induced by small intra-cavity phase anisotropies. Optics Communications, 2009. 282(8): p. 1602-1606. 
75. Adhikari, A. and A. Asundi. Modeling, simulation, and analysis of birefringent effects in plastic optics. 2015.

76. Milby, E., Investigating Stress Birefringence Using Photoelasticity.

77. Dally, J.W. and W.F. Riley, Experimental stress analysis, 1991. McGraw and Hill, New York.

78. Ramesh, K., Digital photoelasticity. 2000, IOP Publishing.

79. Wang, T.-H. and W.-B. Young, Study on residual stresses of thinwalled injection molding. European Polymer Journal, 2005. 41(10): p. 2511-2517.

80. Horn, G., et al., Infrared grey-field polariscope: A tool for rapid stress analysis in microelectronic materials and devices. Review of Scientific Instruments, 2005. 76(4): p. 045108.

81. Pin, W. and A. Asundi, Low birefringence polariscope. 2008.

82. Chidley, M.D., et al. Flow-induced birefringence: the hidden PSF killer in high performance injection-molded plastic optics. in Biomedical Optics 2006. 2006. International Society for Optics and Photonics.

83. Xuehong Lu, L.S.K., A statistical experimental study of the injection molding of optical lenses Journals of Materials Processing Technology 2001. 113(2001): p. 189-175.

84. X. Chena, Y.C.L., D.Q. Lib, Analysis of thermal residual stress in plastic injection molding. Journal of Materials Processing Technology, 2000. 101: p. 275 \pm 280 .

85. Adhikari, A. and A. Asundi. Birefringence characterization of injection molded microplates. 2015.

86. Asundi, W.P.a.A., Full-field retardation measurement of a liquid crystal cell with a phase shift polariscope. Optical Society of America 2008. 47.

87. Guan, L., et al. Spectroscopic imaging ellipsometry for thin film detection on uniaxial crystal. in Fifth International Conference on Optical and Photonics Engineering. 2017. International Society for Optics and Photonics.

88. Taya, S.A. and T.M. El-Agez, Rotating polarizer analyzer ellipsometer with a fixed compensator. Optik-International Journal for Light and Electron Optics, 2013. 124(18): p. 3379-3383.

89. Nagesh, B.V., et al., Birefringence of a normal human red blood cell and related optomechanics in an optical trap. Journal of biomedical optics, 2014. 19(11): p. 115004-115004.

90. New Products. Science, 2004. 306(5699): p. 1199. 
91. Lien, A. and H. Takano, Cell gap measurement of filled twisted nematic liquid crystal displays by a phase compensation method. Journal of Applied Physics, 1991. 69(3): p. 1304-1309.

92. Demos, S.G., H.B. Radousky, and R.R. Alfano, Deep subsurface imaging in tissues using spectral and polarization filtering. Optics Express, 2000. 7(1): p. 23-28.

93. Feldman, A., Measurement of the Photoelastic Contents of Optical Materials. Optical Engineering, 1978. 17(5): p. 175453-175453-.

94. Cloud, G., Optical methods in experimental mechanics. Experimental Techniques, 2010. 34(6): p. 11-14.

95. Yupapin, P. and S. Kusamran, Photoelastic material characteristics based on the stress-induced effect for sensor application. Smart Materials and Structures, 1993. 2(3): p. 157.

96. Feldman, A. Photoelastic properties of optical materials. in 30th Annual Technical Symposium. 1987. International Society for Optics and Photonics.

97. Mueller, H., The theory of photoelasticity. Journal of the American Ceramic Society, 1938. 21(1): p. 27-33.

98. Dixon, R., Photoelastic properties of selected materials and their relevance for applications to acoustic light modulators and scanners. Journal of Applied Physics, 1967. 38(13): p. 5149-5153.

99. Primak, W. and D. Post, Photoelastic constants of vitreous silica and its elastic coefficient of refractive index. Journal of Applied Physics, 1959. 30(5): p. 779-788.

100. Serkowski, K., D. Mathewson, and V. Ford, Wavelength dependence of interstellar polarization and ratio of total to selective extinction. The Astrophysical Journal, 1975. 196: p. 261-290.

101. Chenault, D.B., et al., Snap-shot imaging polarimeter: performance and applications. 2014. 9099: p. 909903.

102. Abrahamsson, S., et al., MultiFocus Polarization Microscope (MFPolScope) for $3 D$ polarization imaging of up to 25 focal planes simultaneously. Optics express, 2015. 23(6): p. 7734-7754.

103. Al-Qasimi, A., et al., Definitions of the degree of polarization of a light beam. Optics letters, 2007. 32(9): p. 1015-1016.

104. Bueno, J.M., et al., Degree of polarization as an objective method of estimating scattering. JOSA A, 2004. 21(7): p. 1316-1321.

105. Azzam, R., Photopolarimetric measurement of the Mueller matrix by Fourier analysis of a single detected signal. Optics Letters, 1978. 2(6): p. 148-150. 
106. Dev, K. and A. Asundi, Mueller-Stokes polarimetric characterization of transmissive liquid crystal spatial light modulator. Optics and Lasers in Engineering, 2012. 50(4): p. 599607.

107. Le Roy-Bréhonnet, F., et al., Optical media and target characterization by Mueller matrix decomposition. Journal of Physics D: Applied Physics, 1996. 29(1): p. 34.

108. Adhikari, A., K. Dev, and A. Asundi, Subwavelength metrological chracterization by Mueller matrix polarimeter and finite difference time domain method. Optics and Lasers in Engineering, 2016. 86: p. 242-247. 


\section{Appendix}

Mueller calculus: Mathematical modelling and analysis in low birefringence measurement system

The stokes vector S' of the light that emerges from the second quarter-wave plate is,

$$
S^{\prime}=P_{0} R_{0}(\delta) Q_{45} P_{0} S
$$

Where $R_{0}(\delta)$ and $Q_{ \pm 45}$ are the Mueller matrices of the specimen and the quarter wave plate whose fast axis makes an angle of $\pm 45^{0}$ with the $O x$ axis. Matrices $\boldsymbol{R}_{\mathbf{0}}(\boldsymbol{\delta})$ and $Q_{ \pm 45}$, obtained by putting $\beta=0$ and $\beta= \pm 45^{\circ}, \delta=90^{\circ}$, are

$$
\begin{aligned}
& \text { Light source represented by stokes vector }\left(\mathrm{S}_{0}\right)=\left[\begin{array}{l}
1 \\
0 \\
0 \\
0
\end{array}\right] \\
& \text { Vertical linear polarizer }\left(\mathrm{P}_{1}\right)=\frac{1}{2}\left[\begin{array}{cccc}
1 & -1 & 0 & 0 \\
-1 & 1 & 0 & 0 \\
0 & 0 & 0 & 0 \\
0 & 0 & 0 & 0
\end{array}\right] \\
& \text { Quarter wave plate }\left(\mathrm{Q}_{\mathrm{w}}\right)=\frac{1}{2}\left[\begin{array}{cccc}
1 & 0 & 0 & -1 \\
0 & 0 & 0 & 0 \\
0 & 0 & 0 & 0 \\
-1 & 0 & 0 & 1
\end{array}\right] \\
& \boldsymbol{R}_{0}(\delta)=\left[\begin{array}{cccc}
1 & 0 & 0 & 0 \\
0 & \cos ^{2} 2 \beta+\sin ^{2} 2 \beta \cos \delta & (1-\cos \delta) \sin 2 \beta \cos 2 \beta & -\sin 2 \beta \sin \delta \\
0 & (1-\cos \delta) \sin 2 \beta \cos 2 \beta & \sin ^{2} 2 \beta+\cos ^{2} 2 \beta \cos \delta & \cos 2 \beta \sin \delta \\
-1 & \sin 2 \beta \sin \delta & -\cos 2 \beta \sin \delta & \cos \delta
\end{array}\right]
\end{aligned}
$$




$$
\text { Analyzer }\left(A_{1}\right)=\frac{1}{2}\left[\begin{array}{cccc}
1 & \cos 2 \theta & \sin 2 \theta & 0 \\
\cos 2 \theta & \cos ^{2} 2 \theta & \sin 2 \theta \cos 2 \theta & 0 \\
\sin 2 \theta & \sin 2 \theta \cos 2 \theta & \sin ^{2} 2 \theta & 0 \\
-1 & 0 & 0 & 0
\end{array}\right]
$$

Thus, stokes vector S' of light that emerges from the quarter wave plate on low birefringence polariscope according to Mueller calculus is given by,

$$
S^{\prime}=P_{0} R_{0}(\delta) Q_{45} P_{0} S
$$

On doing the matrix multiplication via MATLAB we get,

$$
S^{\prime}=\left[\begin{array}{c}
1+\cos 2 \theta \sin 2 \beta \sin \delta-\sin 2 \theta \cos 2 \beta \sin \delta \\
\cos 2 \theta+\cos ^{2} 2 \theta \sin 2 \beta \sin \delta-\cos 2 \theta \sin 2 \theta \cos 2 \beta \sin \delta \\
\sin 2 \theta-\cos 2 \beta \sin ^{2} 2 \theta \sin \delta+\cos 2 \theta \sin 2 \theta \sin 2 \beta \sin \delta \\
0
\end{array}\right]
$$

The intensity of light is represented by the first element in stokes vector which is given by,

$$
I=1+\cos 2 \theta \sin 2 \beta \sin \delta-\sin 2 \theta \cos 2 \beta \sin \delta=1+\sin \delta \sin 2(\beta-\theta)
$$

The obtained equation is similar to the Jones calculus which is modulated at different phase on phase stepping method at $0^{0}, 45^{0}, 90^{0}, 135^{0}$ to obtain phase retardation and direction values. Thus, phase retardation and direction is given by,

$$
\begin{aligned}
& \delta=\sin ^{-1} \frac{\sqrt{\left(I_{3-} I_{1}\right)^{2}+\left(I_{4-} I_{2}\right)^{2}}}{2} \\
& \beta=\frac{1}{2} \tan ^{-1}\left(\frac{I_{1-} I_{3}}{I_{4-} I_{2}}\right)
\end{aligned}
$$


Birefringence measurement results of the optical windows

Circular samples

\begin{tabular}{|llr|}
\hline Sample & Image & Average Birefringence $(\mathrm{nm} / \mathrm{cm})$ \\
1 & FN1_1 & 22.176 \\
3 & FN1_2 & 24.589 \\
4 & FN1_3 & 22.496 \\
5 & FN1_4 & 26.358 \\
6 & FN1_5 & 22.921 \\
7 & FN2_1 & 26.177 \\
8 & FN2_2 & 30.427 \\
9 & FN2_3 & 28.682 \\
10 & FN2_4 & 26.426 \\
11 & FN2_5 & 38.134 \\
12 & FN3_1 & 30.782 \\
13 & FN3_2 & 24.441 \\
14 & FN3_3 & 31.937 \\
15 & FN3_4 & 25.973 \\
16 & FN3_5 & 40.714 \\
17 & FN4_1 & 32.284 \\
18 & FN4_2 & 33.422 \\
19 & FN4_3 & 25.81 \\
20 & FN4_4 & 28.285 \\
& FN4_5 & 34.804 \\
\hline
\end{tabular}




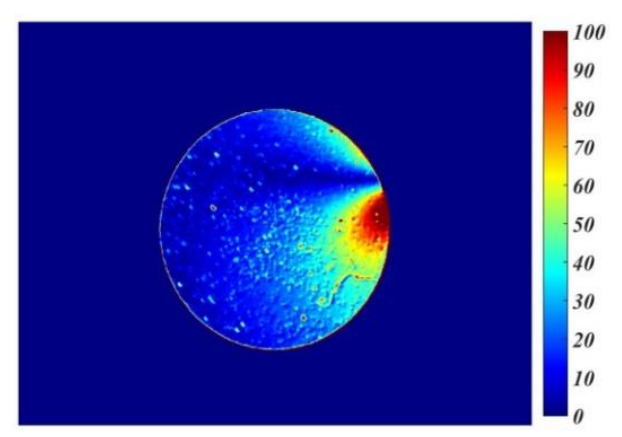

Part No: FN1_1

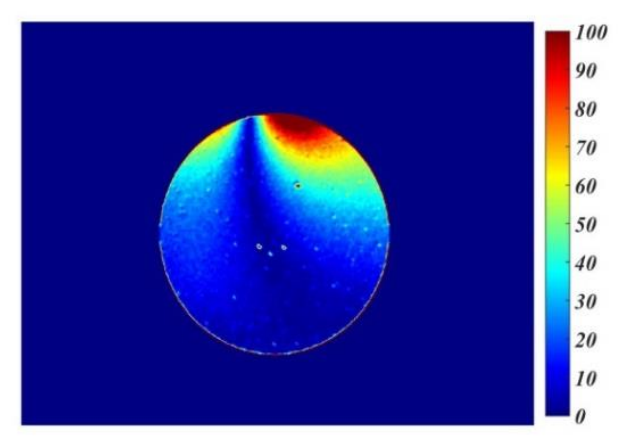

Part No: FN1_3

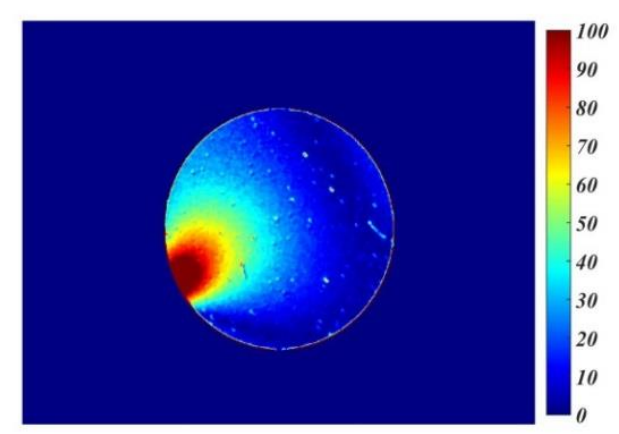

Part No: FN1_2

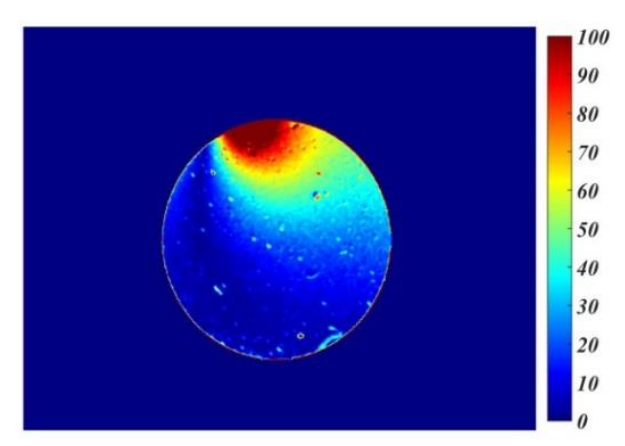

Part No: FN1_4 


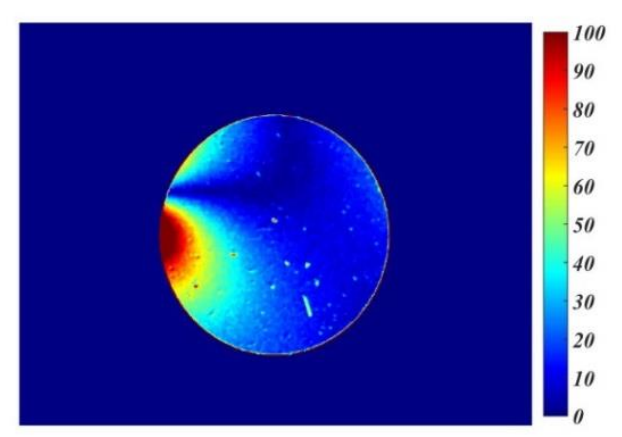

Part No: FN1_5

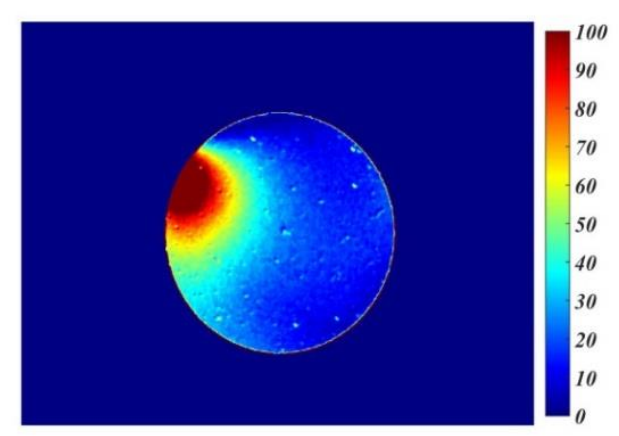

Part No: FN2_2

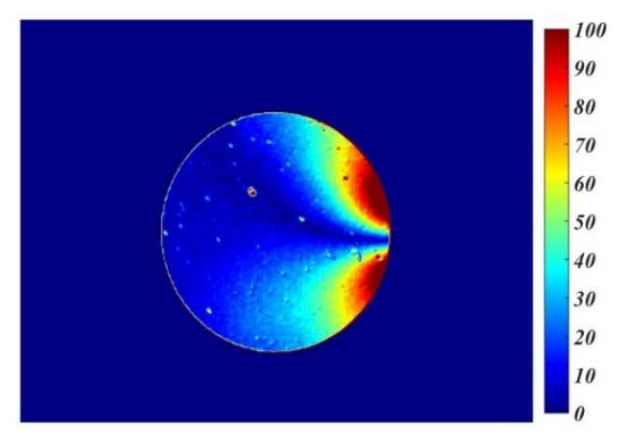

Part No: FN2_1

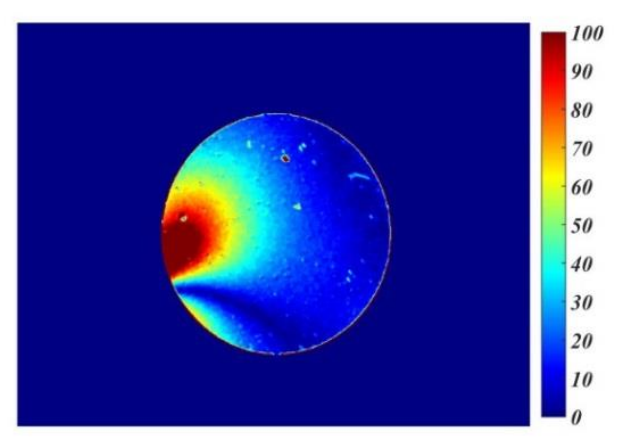

Part No: FN2_3 


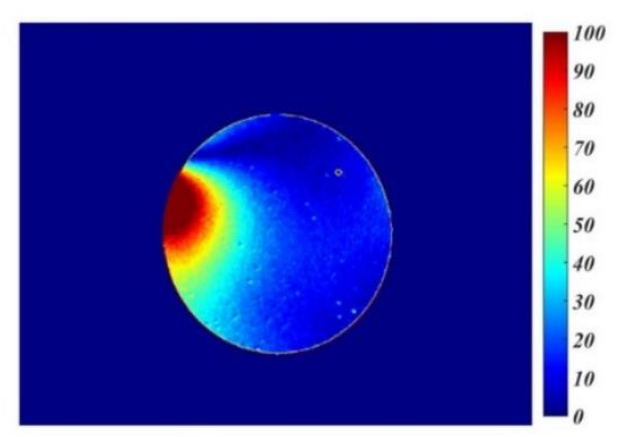

Part No: FN2_4

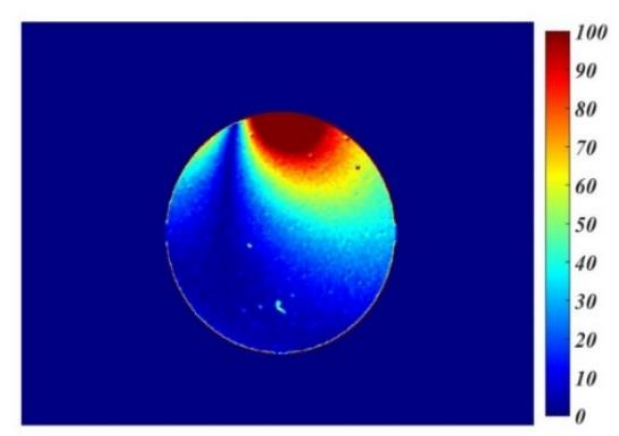

Part No: FN3_1

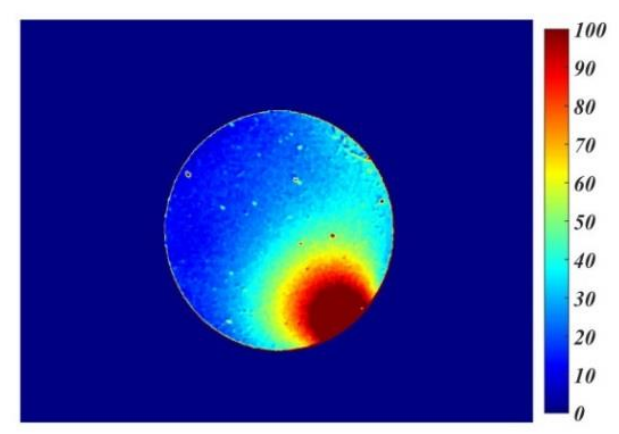

Part No: FN2_5

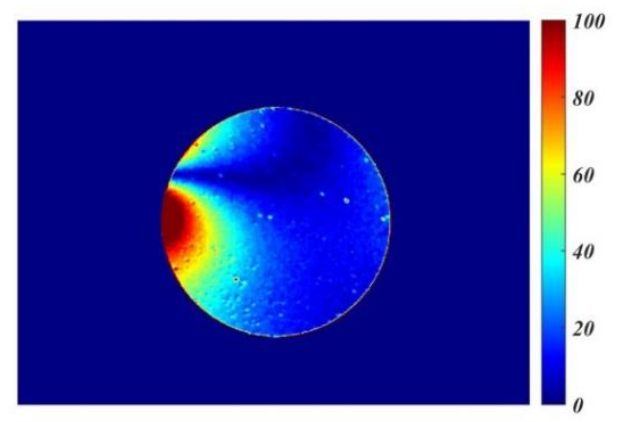

Part No: FN3_2 


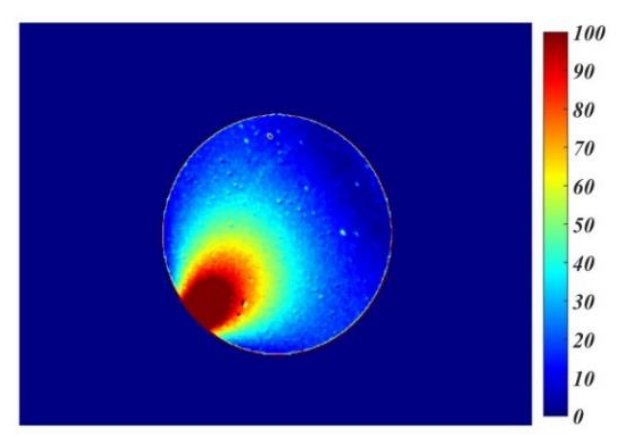

Part No: FN3_3

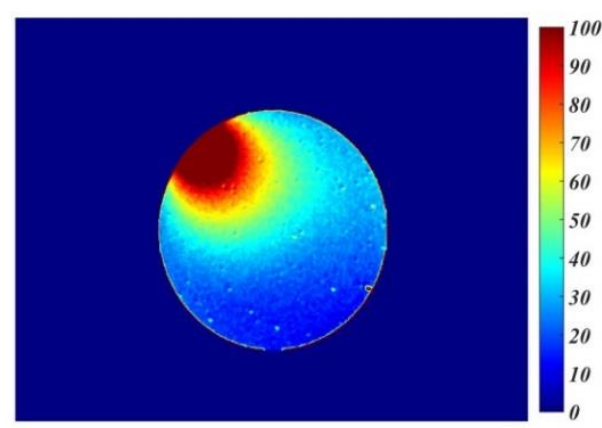

Part No: FN3_5

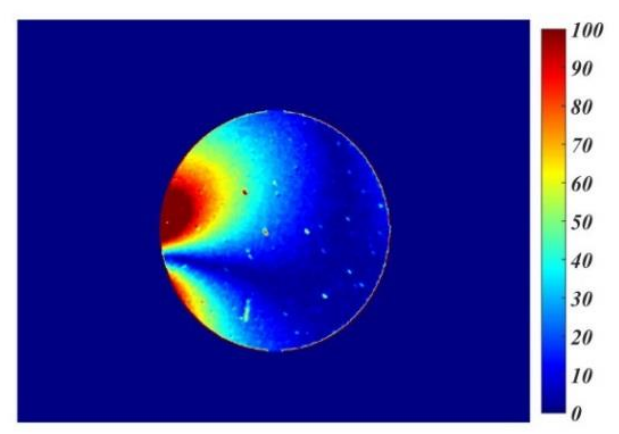

Part No: FN3_4

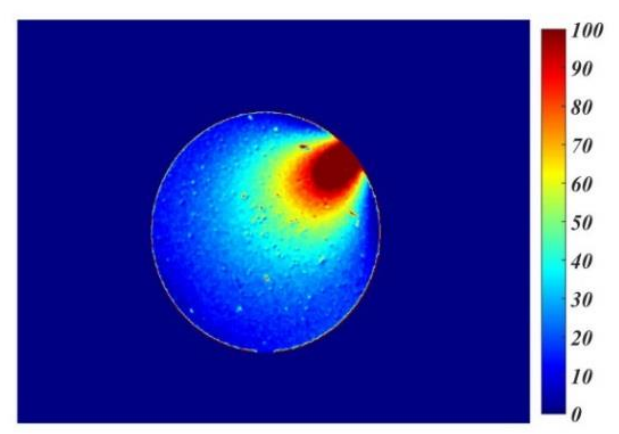

Part No: FN4_1 


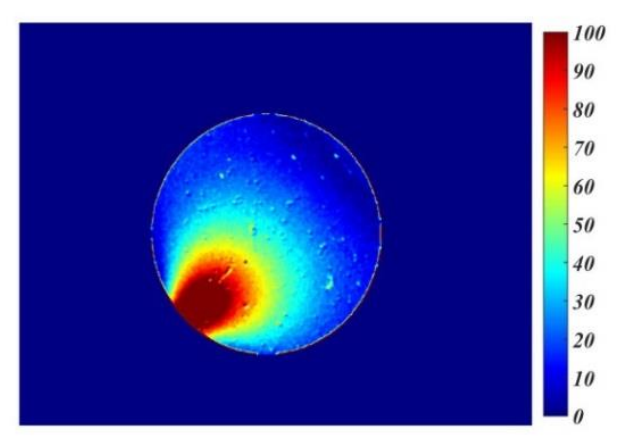

Part No: FN4_2

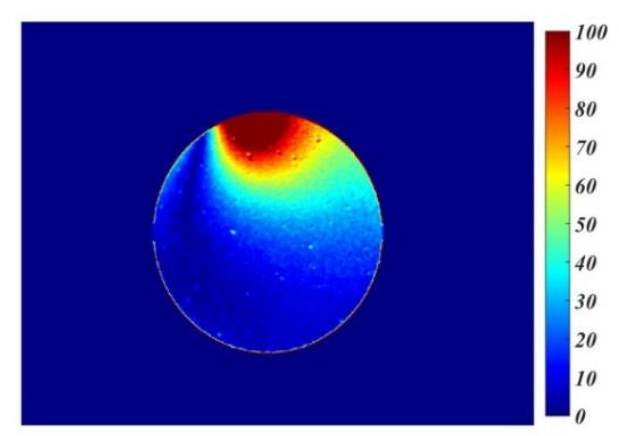

Part No: FN4_4

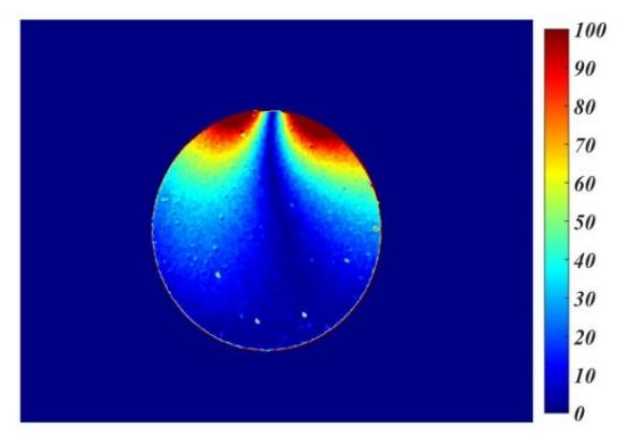

Part No: FN4_3

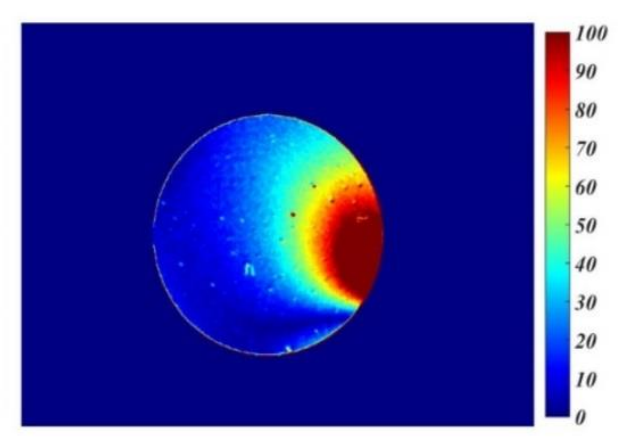

Part No: FN4_5 


\section{Rectangular samples}

\begin{tabular}{|lrr|r|}
\hline Sample & Image & Average Birefringence $(\mathrm{nm} / \mathrm{cm})$ \\
1 & A1_1 & 123.76 \\
2 & A1_2 & 95.947 \\
3 & A1_3 & 126.49 \\
4 & A1_4 & 129.97 \\
5 & A1_5 & 109.68 \\
6 & A1_6 & 106.29 \\
7 & A1_7 & 119.91 \\
8 & A1_8 & 110.88 \\
9 & A1_9 & 113.89 \\
10 & A1_10 & 113.7 \\
11 & B1_1 & 103.68 \\
12 & B1_2 & 126.59 \\
13 & B1_3 & 118.16 \\
14 & B1_4 & 77.162 \\
15 & B1_5 & 113.31 \\
16 & B1_6 & 143.04 \\
17 & B1_7 & 125.44 \\
18 & B1_8 & 127.96 \\
19 & B1_9 & 113.86 \\
20 & B1_10 & 115.23 \\
\hline
\end{tabular}
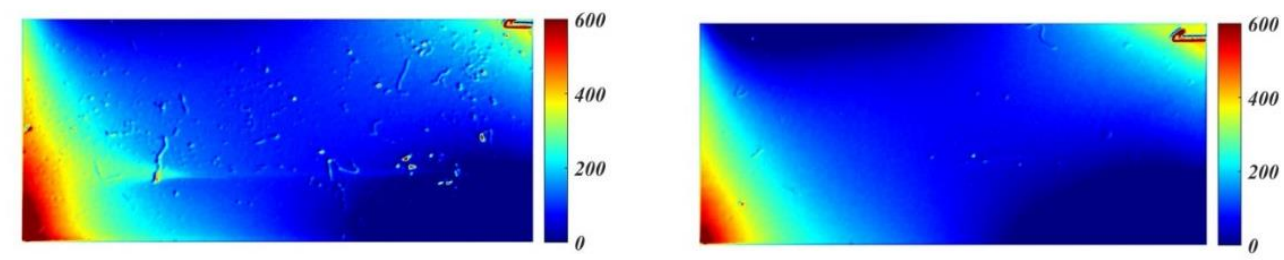

Part No: A1_1

Part No: A1_2 


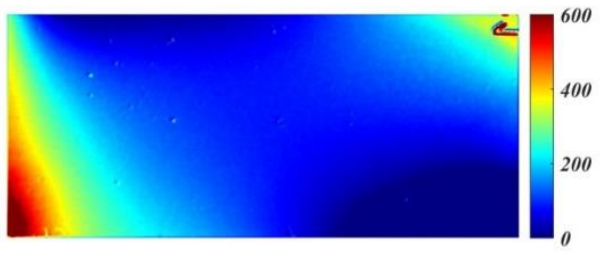

Part No: A1_3

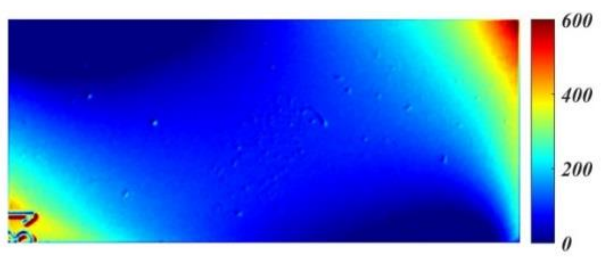

Part No: A1_5

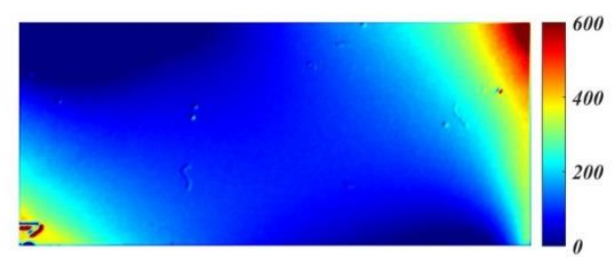

Part No: A1_7

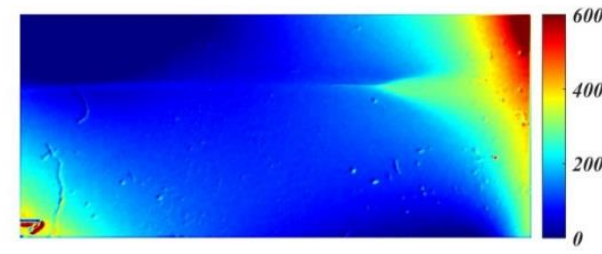

Part No: A1_4

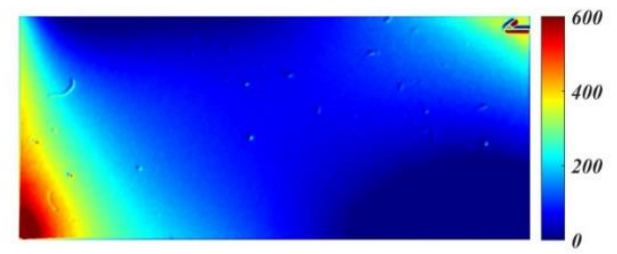

Part No: A1_6

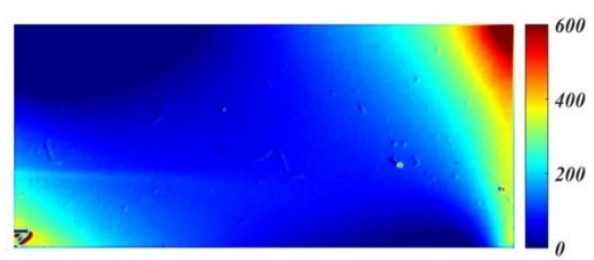

Part No: A1_8 


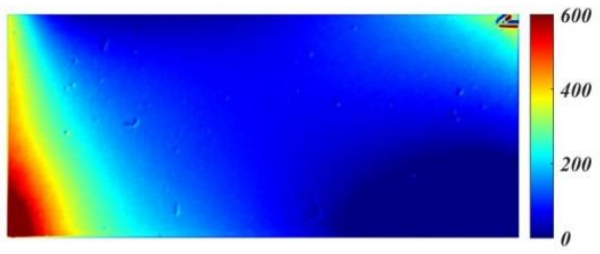

Part No: A1_9

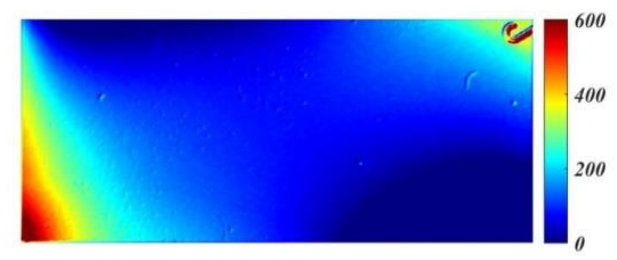

Part No: B1_1

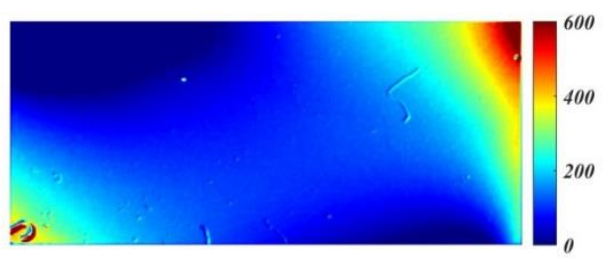

Part No: B1_3

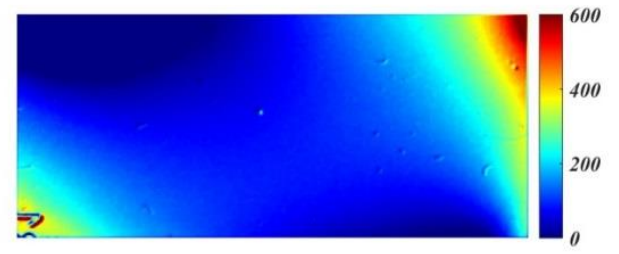

Part No: A1_10

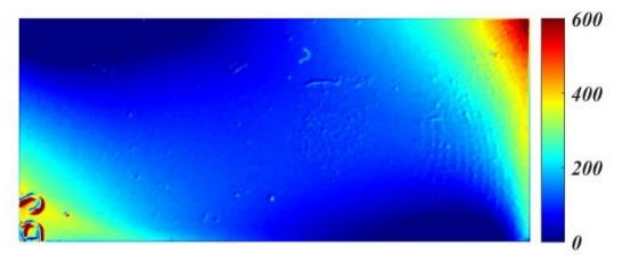

Part No: B1_2

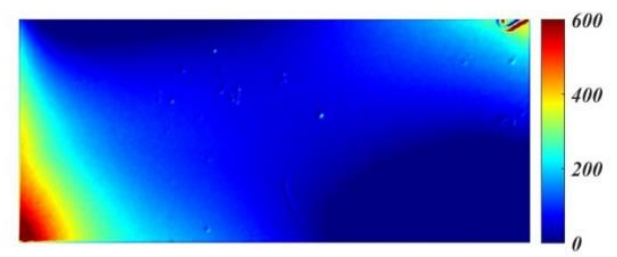

Part No: B1_4 


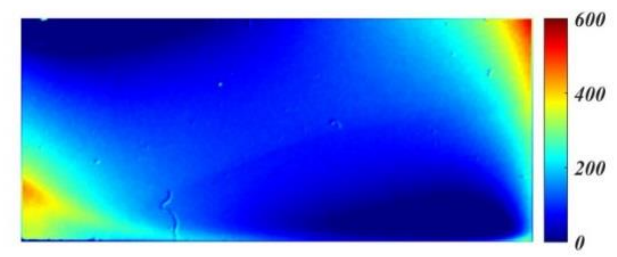

Part No: B1_5

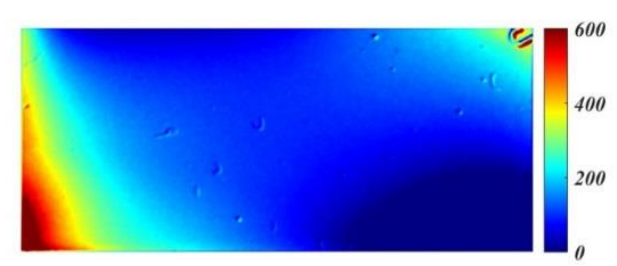

Part No: B1_7

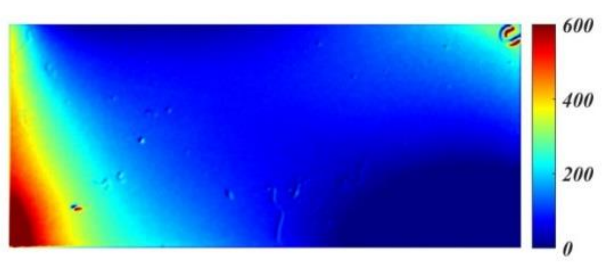

Part No: B1_9

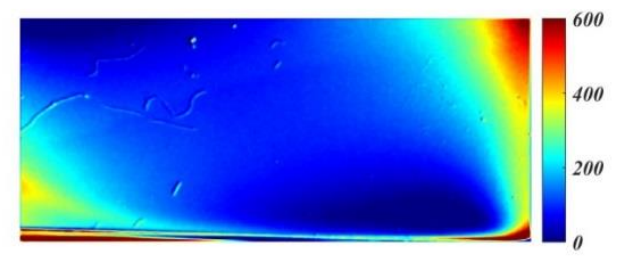

Part No: B1_6

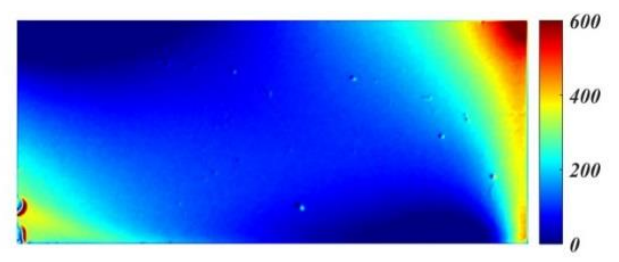

Part No: B1_8

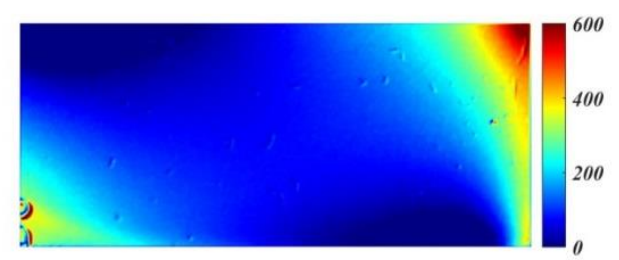

Part No: B1_10 